\title{
ON THE DECOMPOSITION OF LANGLANDS SUBREPRESENTATIONS FOR A GROUP IN THE HARISH-CHANDRA CLASS
}

\author{
EUGENIO GARNICA VIGIL
}

\begin{abstract}
When a group $G$ is in the Harish-Chandra class, the goal of classifying its tempered representations and the goal of decomposing the Langlands subrepresentation for any of its standard representations are equivalent. The main result of this work is given in Theorem (5.3.5) that consists of a formula for decomposing any Langlands subrepresentation for the group $G$. The classification of tempered representations is a consequence of this theorem (Corollary (5.3.6)).
\end{abstract}

\section{INTRODUCTION AND NOTATION}

1.1 Introduction. Admissible representations were introduced by HarishChandra in order to study spaces of functions on a homogeneous space for a real semi-simple Lie group. The main example of admissible representations is given by the irreducible unitary representations of the group. Most of the work of Harish-Chandra is valid not only for real semisimple Lie groups but for groups in the Harish-Chandra class; groups we will be working with (Definition (1.2.2)).

The main objects appearing in this paper are the admissible representations of a group in the Harish-Chandra class $G$. Let $g$ be the complexification of the Lie algebra of the group $G$ and let $K$ be a maximal compact subgroup. The connection between admissible representations of $G$ and $(\mathfrak{g}, K)$-modules is given by the Harish-Chandra correspondence, explained in (4.1.5). Theorem (4.1.7) provides the properties of the correspondence that make apparent the importance of the analysis of the category of $(\mathfrak{g}, K)$-modules.

One of the strongest results achieved in the representation theory of the group $G$ is the Langlands classification, carried out by Langlands, Knapp, Zuckerman and Miličic, mainly. This consists in the classification of all irreducible admissible representations of the group. (In contrast, the problem of classifying its irreducible unitary representations remains open.) The formulation of such classification in terms of real parabolic induction and tempered representations, for a group $G$ in the Harish-Chandra class, is given as follows (see [K] for this):

Received by the editors February 10, 1994.

1991 Mathematics Subject Classification. Primary 22E43, 22E46.

Key words and phrases. Langlands subrepresentations, irreducible tempered representations, Harish-Chandra modules, Vogan-Zuckerman cohomological induction. 
For a fixed minimal parabolic subgroup $P=M A N$, the Langlands classification establishes a one-to-one correspondence between equivalence classes of irreducible admissible representations and all triples $\left(P_{1},\left[\sigma_{1}\right], \nu_{1}\right)$ such that

(a) $P_{1}=M_{1} A_{1} N_{1}$ is a parabolic subgroup of $G$ and $P \subseteq P_{1}$.

(b) $\left[\sigma_{1}\right]$ is the class of an irreducible tempered representation $\sigma_{1}$ for $M_{1}$.

(c) $\exp \left(\nu_{1}\right)$ is a non-unitary representation of $A_{1}$ such that $\left(-\operatorname{Re}\left(\nu_{1}\right)\right)$ is in the open Weyl chamber determined by $N_{1}$.

The correspondence associates to an irreducible admissible representation $X$ a triple $\left(P_{1},\left[\sigma_{1}\right], \nu_{1}\right)$ such that $X$ is the unique irreducible subrepresentation of $G$ contained in the induced module $\operatorname{Ind}_{P_{1}}^{G}\left(\sigma_{1} \otimes \nu_{1} \otimes 1\right)$.

The definition of a tempered representation can be found in [K] and [W2]; however, for the sake of exposition, we adopt the definition of tempered given in (4.1.17). The reason to do this is the fact, due to Harish-Chandra, that any irreducible tempered representation of $G$ is the subrepresentation of one unitarily induced from discrete series. We insert this in the Langlands classification above.

The representations $\operatorname{Ind}_{P_{1}}^{G}\left(\sigma_{1} \otimes \nu_{1} \otimes 1\right)$ above are called final. For a parabolic subgroup $P_{2}=M_{2} A_{2} N_{2}$, a standard representation is an induced representation Ind $_{P_{2}}^{G}\left(\sigma_{2} \otimes \nu_{2} \otimes 1\right)$ where $\sigma_{2}$ is a discrete series representation of $M_{2}$ and $\left(-\operatorname{Re}\left(\nu_{2}\right)\right)$ is in the closed Weyl chamber determined by $N_{2}$. As an application of parabolic induction by stages, we note in (4.1.21) that a final representation is a subrepresentation of some standard, and each standard representation can be decomposed as a direct sum of final representations.

While the maximal completely reducible subrepresentation, the Langlands subrepresentation, of a final representation is irreducible, that of a standard representation might have a proper subrepresentation in general. Thus, using the Langlands disjoint theorem (see [K] and [Green]), which asserts that an irreducible admissible representation cannot be a direct summand of two differnt standard representations, the Langlands classification implies that the set of irreducible admissible representations is the disjoint union of Langlands packets, each of which consists of the irreducible constituents that appear in the direct sum decomposition of the Langlands subrepresentation of some standard representation.

What makes the study of standard representations more accessible than final ones is due to the fact that discrete series are better known than tempered representations. We observe in (4.1.21), applying induction by stages, that classifying tempered representations is equivalent to knowing the decomposition of all the Langlands subrepresentations. The main result of this paper is Theorem (5.3.5), which provides a formula for the decomposition of the Langlands subrepresentation of a standard representation when the group $G$ is in the Harish-Chandra class. The classification of tempered representations is an easy consequence (Corollary (5.3.6)).

A formula for the decomposition of the Langlands subrepresentations is an important step toward solving the Kazhdan-Lusztig conjecture for the category of admissible representations. The solution of this conjecture is to find an algorithm to describe the composition series for any standard representation.

As for the classification of tempered representations, its value is evident just by the Langlands classification; however, we should underline the significant 
role tempered representations play in Harish-Chandra's monumental work. In [H-Ch 3] Harish-Chandra approaches somewhat to a classsification theorem for them. He seems to include there (Theorem 8) the fact that all irreducible constituents of an induced representation from discrete series have the same multiplicity.

The classification of irreducible tempered representations was achieved by Knapp and Zuckerman in [K-Z] for linear reductive Lie groups. Mirković in [M] extends this result for non-linear groups in the language of $D$-modules. Our description of the classification differs from Mirković's; it follows Vogan's approach.

One common tool used in the sources above and in the Vogan calculus is the Knapp-Stein theory of the $R$-group.

In a different setting, we find once more that tempered representations play a leading role. They appear in Arthur's work involved in his trace formulas. In the unpublished paper [A], Arthur studies elliptic tempered representations. Here, by means of the $R$-group, he provides a classification of irreducible tempered characters for a connected reductive algebraic group over a local field of characteristic zero. However, discrete series for $p$-adic groups, $R$-groups, central extensions of $R$-groups cannot be explicitly computed as in the real case.

As mentioned, we use Vogan's method. By means of the Harish-Chandra correspondence and the Vogan-Zuckerman theory of cohomological induction, in [Green] Vogan provides a formula for the decomposition of any Langlands subrepresentation of a general standard representation for a linear group, reducing the problem strikingly to study fine representations (section 2). Either for a linear or connected real reductive Lie group, he defines an action of the $R$-group associated to a standard representation on the set of its minimal $K$ types in the special situation when these are fine. At this level the multiplicity of any minimal $K$-type and any irreducible representation appearing in the Langlands subrepresentation is one. It turns out that the proper Zuckerman functors applied to induce these to standard representations of $G$ preserve this multiplicity-one property (5.1.7).

As shown in Example (1.1.1) below, the multiplicity-one property fails for a group in the Harish-Chandra class. To compute multiplicities, the main obstacle to overcome in generalizing Vogan's formula is the lack of a natural extension to the case of non-linear groups of the action of the $R$-group $R_{\delta}$ for standard representations $\operatorname{Ind}_{P}^{G}(\delta \otimes 1)$ given in [Green] when $\delta$ is a fine $M$-representation. In Lemma (4.3.3) we are able to construct an action not of the $R$-group $R_{\delta}$ but rather of its group of characters $\widehat{R_{\delta}}$ on the set of $\delta$-primary parts of the minimal $K$-types that are fine, $A_{d}(\delta)$. Then, this can be extended on the set of minimal $K$-types $A(\delta)$ because of the bijection between $A_{d}(\delta)$ and $A(\delta)$. This provides a transitive action on $A(\delta)$ and on the irreducible constituents in the Langlands subrepresentation of $\operatorname{Ind}_{P}^{G}(\delta \otimes 1)$. Moreover, since Zuckerman functors behave very well, we can extend this new action to the general sitution.

We observe that in order to compute multiplicities it is enough to reduce the problem not only to the setting where minimal $K$-types are fine but to the special situation when the group $G$ is basically a direct product of copies of $S l(2, \mathbb{R})$. To be more precise, see Example (4.3.1).

Example 1.1.1. There exists a group $G$ in the Harish-Chandra class such that its identity component $G_{o}$ is given by 


$$
G_{o}=\frac{(S l(2, \mathbb{R}) \times S l(2, \mathbb{R}))}{\left\{ \pm\left(\begin{array}{ll}
I & 0 \\
0 & I
\end{array}\right)\right\}}
$$

and if $P=M A N$ is a minimal parabolic subgroup of $G$ then $M$ is isomorphic to the quaternion group. Write $M^{o}:=M \cap G_{o}$; hence $M^{o} \cong \mathbb{Z} / 2 \mathbb{Z}$. Consequently, we have $G / G_{o} \cong M / M^{o} \cong \mathbb{Z} / 2 \mathbb{Z}+\mathbb{Z} / 2 \mathbb{Z}$. Let $\delta$ be the twodimensional representation of $M$. It is known that this representation restricted to $M^{o}$ can be written as the direct sum $\left.\delta\right|_{M^{o}} \cong \delta_{o}+\delta_{o}$ where $\delta_{o}$ is the nontrivial representation of $\mathbb{Z} / 2 \mathbb{Z}$; that is, we have the multiplicity $m\left(\delta_{o}, \delta\right)=2$. Using Lemma (4.3.2), we conclude that $\operatorname{Ind}_{P}^{G}(\delta \otimes 1) \cong I+I$ where $I$ is an irreducible representation of $G$ appearing with multiplicity two; there is only one minimal $K$-type in the standard representation appearing with multiplicity two.

The decomposition in Theorem (5.3.5) is formulated in terms of representations which are cohomologically induced from pseudo-characters, which are generalization of regular characters. Standard representations correspond precisely to regular characters (see [Green]). A result in [A-B-V] asserts that any representation induced from a final character, (5.1.13), is non-zero and indecomposable. On the other hand, applying Schmid identities to a standard representation we can decompose it as direct sum of representations induced from final characters. Although the pseudo-characters that we find to decompose a Langlands subrepresentation in Theorem (5.3.5) are not necessarily final, the representations they give rise are indecomposable. This means that each of this representations is isomorphic to one induced from a final character.

This paper contains the results obtained in my doctoral thesis at M.I.T. I would like to thank David Vogan, my thesis supervisor, for having suggested this subject to me, for his patience during many discussions, and for having given me some suggestions for the improvement of this manuscript.

1.2 Notation. We will use the following notation coming from [Green]:

Lie groups are denoted by upper case italic letters such as $G, H, P, L, \ldots$, and complex Lie algebras are denoted by German letters such as $\mathfrak{g}, \mathfrak{k}, \mathfrak{u}, \ldots$.

$\operatorname{Lie}(G)$ is used to mean the real Lie algebra of the Lie group $G$. Real Lie algebras are denoted by German letters with subscript $o$, and their complexifications are denoted by the same letters without the subindex $o$. Example: $\mathfrak{g}_{o}=\operatorname{Lie}(G)$ and $\mathfrak{g}=\mathfrak{g}_{o} \otimes \mathbb{C}$.

For a Lie group $L, L_{o}$ will denote the identity component. If $\mathfrak{g}$ is a reductive Lie algebra with a Cartan subalgebra $\mathfrak{h}, \Delta(\mathfrak{g}, \mathfrak{h})$ will denote the set of roots of $\mathfrak{h}$ in $\mathfrak{g}$. More generally, if $V$ is the representation of an abelian Lie algebra, $\Delta(V)=\Delta(V, \mathfrak{h})$ will denote the set of weights of $\mathfrak{h}$ in $V$. For $\Delta(V)$, we write

(sum with multiplicities)

$$
\rho(V)=\rho(\Delta(V))=\frac{1}{2}\left(\sum_{\lambda \in \Delta(V)} \lambda\right)
$$

Now, to describe the type of groups we will be working with, suppose $G_{\mathbb{C}}$ is a connected reductive algebraic group over $\mathbb{C}$. In this case, denote $\mathfrak{g}=\operatorname{Lie}\left(G_{\mathbb{C}}\right)$. Definition 1.2.1 [A-B-V]. An antiholomorphic involutive automorphism

$$
\sigma: G_{\mathbb{C}} \rightarrow G_{\mathbb{C}}
$$

will be called a real form of $G_{\mathbb{C}}$. 
We get an antiholomorphic involutive automorphism of $\mathfrak{g}$ by $d \sigma: \mathfrak{g} \rightarrow \mathfrak{g}$, which is the differential of a real form $\sigma$ of $G$. Denote

$$
\begin{aligned}
G(\mathbb{R}, \sigma) & =\left\{g \in G_{\mathbb{C}} \mid \sigma(g)=g\right\}, \\
\mathfrak{g}(\mathbb{R}, \sigma) & =\{X \in \mathfrak{g} \mid d \sigma(X)=X\} .
\end{aligned}
$$

When $G(\mathbb{R}, \sigma)$ is a real compact Lie group, $\sigma$ is called a compact real form for $G_{\mathbb{C}}$. Every connected reductive algebraic group $G_{\mathbb{C}}$ over $\mathbb{C}$ has a compact real form that we denote by $\sigma_{c}$.

Definition 1.2.2. A real Lie group $G$ is said to be in the Harish-Chandra class if we have

(a) a connected reductive algebraic group $G_{\mathbb{C}}$ over $\mathbb{C}$,

(b) a real form $\sigma$ of $G_{\mathbb{C}}$ commuting with $\sigma_{c}$,

(c) a homomorphism $\varphi: G \rightarrow G(\mathbb{R}, \sigma)$ having finite kernel and finite cokernel,

(d) $\operatorname{Ad}(G) \subseteq \operatorname{Ad}\left(G_{\mathbb{C}}\right)$.

We will identify $\mathfrak{g}_{o}=\operatorname{Lie}(G)$ with $\mathfrak{g}(\mathbb{R}, \sigma)$ and $\mathfrak{g}_{o} \otimes \mathbb{C}$ with $\mathfrak{g}$ through $\varphi$.

Suppose $G$ is in the Harish-Chandra class with data $\left(G_{\mathbb{C}}, \sigma, \varphi\right)$ as in definition (1.2.2). Consider the Cartan involution $\theta=\sigma \cdot \sigma_{c}$. We get a maximal compact subgroup $K$ for $G$, a Cartan decomposition $G=K \times \exp \left(\mathfrak{p}_{o}\right)$ and an Iwasawa decomposition $G=K A N$ by transferring under the inverse image of $\varphi$ the corresponding structure from $G(\mathbb{R}, \sigma)$. Every parabolic subgroup $P_{1}$ of $G$ is the inverse image under $\varphi$ of a parabolic subgroup of $G(\mathbb{R}, \sigma)$. So $\varphi^{-1}$ gives us a Langlands decomposition for $P_{1} ; P_{1}=M_{1} A_{1} N_{1}$.

An important point is that $\varphi^{-1}$ applied to a Harish-Chandra subgroup of $G(\mathbb{R}, \sigma)$ is automatically a Harish-Chandra subgroup of $G$; for instance, $M_{1} A_{1}$ and $M_{1}$ are in the Harish-Chandra class. For a Cartan decomposition $G=$ $K \times \exp \left(\mathfrak{p}_{o}\right), T_{1} A_{1}$ will denote the corresponding Cartan decomposition for a $\theta$-stable Cartan subgroup $H_{1} \quad\left(H_{1} \cap K=T_{1}\right)$.

Write $G^{\#}=\operatorname{Ad}^{-1}\left(\operatorname{Ad}\left(G_{o}\right)\right)$, and for any $S \subseteq G, S^{\#}$ will stand for $S \cap G^{\#}$.

For any compact Lie group $C$ and any Cartan subgroup $H, \widehat{C}$ and $\widehat{H}$ will denote the corresponding sets of isomorphism classes of irreducible representations.

Suppose $B \subseteq A$, and $\delta \in \widehat{B}$ and $\mu \in \widehat{A} . m(\delta, \mu)$ will denote the multiplicity of $\delta$ in the restriction $\left.\mu\right|_{B}$.

\section{II. $M$-FINE REPRESENTATIONS AND $K$-FINE REPRESENTATIONS}

(2.1) Let $G$ be a Lie group in the Harish-Chandra class (Definition (1.2.2)), with a Cartan decomposition $G=K \times \exp \left(\mathfrak{p}_{o}\right)$ and with Cartan involution $\theta$. The complexification of the Lie algebras $\mathfrak{g}_{o}=\operatorname{Lie}(G)$ and $\mathfrak{k}_{o}=\operatorname{Lie}(K)$ are denoted by $\mathfrak{g}=\mathfrak{g}_{o} \otimes \mathbb{C}$ and $\mathfrak{k}=\mathfrak{k}_{o} \otimes \mathbb{C}$.

Write $\mathfrak{p}=\mathfrak{p}_{o} \otimes \mathbb{C}$; thus, $\mathfrak{g}=\mathfrak{k}+\mathfrak{p}$. Let $\mathfrak{h}$ be a $\theta$-stable Cartan subalgebra of $\mathfrak{g}$. The root system $\Delta=\Delta(\mathfrak{g}, \mathfrak{h})$ gives rise to the following root space decomposition:

$$
\mathfrak{g}=\mathfrak{h}+\sum_{\alpha \in \Delta} \mathfrak{g}_{\alpha} .
$$

There is a natural action of $\theta$ on $\Delta(\mathfrak{g}, \mathfrak{h})$. This yields different types of roots. 
Definition 2.2. For a $\theta$-stable Cartan subalgebra $\mathfrak{h}$ of $\mathfrak{g}$, we call a root $\alpha \in$ $\Delta(\mathfrak{g}, \mathfrak{h})$

(a) real if $\theta \cdot \alpha=-\alpha$,

(b) imaginary if $\theta \cdot \alpha=\alpha$,

(i) compact imaginary if $\theta \cdot \alpha=\alpha$ and $\mathfrak{g}_{\alpha} \subseteq \mathfrak{k}$,

(ii) noncompact imaginary if $\theta \cdot \alpha=\alpha$ and $\mathfrak{g}_{\alpha} \subseteq \mathfrak{p}$,

(c) complex if $\theta \cdot \alpha \neq \pm \alpha$.

(2.3) (a) Let $\mathfrak{a}_{o}$ be a maximal abelian subalgebra of $\mathfrak{g}_{o}$ contained in $\mathfrak{p}_{o}$ with complexification $\mathfrak{a}$ and underlying subgroup $A$ of $G$ (i.e., $\left.\mathfrak{a}_{o}=\operatorname{Lie}(A)\right)$. Set

$$
\begin{aligned}
& M=\text { centralizer of } \mathfrak{a}_{o} \text { in } K, \\
& M^{\prime}=\text { normalizer of } \mathfrak{a}_{o} \text { in } K .
\end{aligned}
$$

(b) Consider a $\theta$-stable Cartan subalgebra $\mathfrak{h}^{s}$ containing $\mathfrak{a}_{o}$. Such an algebra is called maximally split. We define two sets of restricted roots:

$$
\begin{aligned}
\bar{\Delta}_{r} & =\left\{\left.\alpha\right|_{\mathfrak{a}}|\alpha|_{\mathfrak{a}} \neq 0 \text { and } \alpha \in \Delta\left(\mathfrak{g}, \mathfrak{h}^{s}\right)\right\}, \\
\bar{\Delta} & =\left\{\alpha \in \bar{\Delta}_{r} \mid \frac{1}{2} \alpha \notin \bar{\Delta}_{r}\right\} .
\end{aligned}
$$

Here we mention some important results involving $\bar{\Delta}$, found in $([\mathrm{H}]$, Chapter VI). Both sets $\bar{\Delta}_{r}$ and $\bar{\Delta}$ are root systems. $\bar{\Delta}$ is in fact a reduced root system and its Weyl group $W(\bar{\Delta})$ can be identified with $W=M^{\prime} / M$. Iwasawa decompositions of $G$ containing $K A$ are in one-to-one correspondence with minimal parabolic subgroups containing $M A$ and these, in turn, are in one-to-one correspondence with positive systems for $\bar{\Delta}$.

Definition 2.4. Let $\alpha$ be a root in the reduced root system $\bar{\Delta}$, defined in (2.3).

(a) It is called real if $\alpha$ is the restriction of some real root of $\Delta$.

(b) It is complex if $\alpha$ is not real.

(2.5) Let $G$ be a group in the Harish-Chandra class (1.2.2) with data $\left(G_{\mathbb{C}}, \sigma\right.$, $\varphi)$. Now we know, there is a non-trivial homomorphism $S L(2, \mathbb{R}) \stackrel{\Phi_{\alpha}}{\longrightarrow}$ $G(\mathbb{R}, \sigma)$ for any real root $\alpha \in \bar{\Delta}$ such that $\Phi_{\alpha}$ (diagonal matrices) $\subseteq H_{\mathbb{R}}^{s}$, $\phi_{\alpha}\left(\left(\begin{array}{ll}0 & 1 \\ 0 & 0\end{array}\right)\right) \in \mathfrak{g}_{\alpha}$, and $\phi_{\alpha}$ carries inverse transpose of $\operatorname{sl}(2, \mathbb{R})$ to the Cartan involution of $G$. Here $H_{\mathbb{R}}^{s}$ is the Cartan subgroup of $G(\mathbb{R}, \sigma)$ corresponding to $\mathfrak{h}_{o}^{s}$ and $d \Phi_{\alpha}=\phi_{\alpha}$. Moreover, we have the following commutative diagram:

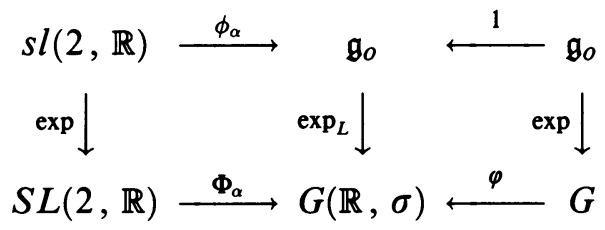

where $\exp _{L}$ is the exponential map in the linear group. We put

$$
Z_{\alpha}=\phi_{\alpha}\left(\left(\begin{array}{ll}
0 & 1 \\
0 & 0
\end{array}\right)\right), \quad \sigma_{\alpha}^{\prime}=\Phi_{\alpha}\left(\left(\begin{array}{cc}
0 & 1 \\
-1 & 0
\end{array}\right)\right)=\exp _{L}\left(\frac{\pi}{2} Z_{\alpha}\right)
$$

and

$$
m_{\alpha}^{\prime}=\left(\sigma_{\alpha}^{\prime}\right)^{2}=\Phi_{\alpha}\left(\left(\begin{array}{cc}
-1 & 0 \\
0 & -1
\end{array}\right)\right)
$$


Lemma 2.6 ([Green], p. 172). For the elements defined in (2.5), the map $\phi_{\alpha}$ is unique up to conjugation by $\left(\begin{array}{cc}-1 & 0 \\ 0 & 1\end{array}\right)$ on $\operatorname{sl}(2, \mathbb{R}) ;$ hence, $Z_{\alpha}$ and $\sigma_{\alpha}^{\prime}$ are well defined up to sign and inverse respectively. Furthermore,

(a) $\left(m_{\alpha}^{\prime}\right)^{2}=1$ and $m_{\alpha}^{\prime} \in \varphi(M)$.

(b) $\sigma_{\alpha}^{\prime} \in \varphi\left(M^{\prime}\right)$, and the image of $\overline{\sigma_{\alpha}^{\prime}}$ of $\sigma_{\alpha}^{\prime}$ in $M^{\prime} / M$ is the reflexion $s_{\alpha}$ in $W$.

We are interested in the image of the exponential not in $G(\mathbb{R}, \sigma)$ but rather in $G$. Thus, we define

Definition 2.7. For any real root $\alpha \in \bar{\Delta}$ denote

$$
\sigma_{\alpha}=\exp \left(\frac{\pi}{2} Z_{\alpha}\right) \text { and } m_{\alpha}=\left(\sigma_{\alpha}\right)^{2}
$$

So $\sigma_{\alpha} \in M^{\prime}$ and $m_{\alpha} \in M$.

Definition 2.8. Let $A$ and $M$ be as in Definition (2.3).

Let $H^{s}=T A$ be the Cartan subgroup of $G$ corresponding to $\mathfrak{h}_{o}^{s}=\mathfrak{t}_{o}+\mathfrak{a}_{o}$. $G$ is called quasisplit if $M=T$.

(2.9) Let $\theta$ the Cartan involution of $G$ (or $\mathfrak{g}$ ). The subgroup $\{1, \theta\}$ of $\operatorname{Aut}(\mathfrak{g})$, denoted by $\Gamma$, acts naturally on both $\Delta=\Delta\left(\mathfrak{g}, \mathfrak{h}^{\mathfrak{s}}\right)$ and $\mathfrak{g}$. For every root $\alpha \in \Delta$, note $\theta \cdot \mathfrak{g}_{\alpha}=\mathfrak{g}_{\theta \alpha}$. Suppose $G$ is quasisplit. Thus, $\Delta\left(\mathfrak{g}, \mathfrak{h}^{s}\right)$ does not contain imaginary roots, and for any root $\alpha \in \Delta, \mathfrak{g}_{\alpha}+\mathfrak{g}_{\theta \alpha}$ is an irreducible subspace of $\mathfrak{g}$ under the action of $\Gamma$. Write $[\beta]$ for the set $\{\beta, \theta \beta\}$ for any root $\beta \in \Delta$, and $\Delta / \Gamma=\{[\alpha] \mid \alpha \in \Delta\}$. Therefore, we have the following decompositions:

$$
\mathfrak{g}=\mathfrak{h}^{s}+\left(\sum_{[\alpha] \in \Delta / \Gamma} \mathfrak{g}_{\alpha}+\mathfrak{g}_{\theta \alpha}\right)
$$

and

$$
\mathfrak{k}=\mathfrak{t}^{s}+\sum_{[\alpha] \in \Delta / \Gamma, \theta \alpha=-\alpha} \mathbb{C} Z_{\alpha}+\sum_{[\alpha] \in \Delta / \Gamma, \theta \alpha \neq \pm \alpha} \mathfrak{k}_{\alpha}
$$

where $Z_{\alpha}$ is defined in (2.5) for a real root $\alpha$. There is no ambiguity in the decomposition since by Lemma (2.6), $Z_{\alpha}=-Z_{-\alpha}$. The set $\mathfrak{k}_{\beta}$ is the onedimensional space $\mathfrak{k} \cap\left(\mathfrak{g}_{\beta}+\mathfrak{g}_{\theta \beta}\right)$ given for a complex root $\beta$.

Assume from now to the end of this section that $G$ is quasisplit, (2.8). We consider the decomposition $\mathfrak{g}=[\mathfrak{g}, \mathfrak{g}]+\mathfrak{z}$. Write $\mathfrak{m}^{s}=\mathfrak{m} \cap([\mathfrak{g}, \mathfrak{g}])(\mathfrak{m}=$ $\operatorname{Lie}(M) \otimes \mathbb{C})$. Therefore, we have $\mathfrak{m}=\mathfrak{m}^{s}+(\mathfrak{z} \cap \mathfrak{m})$.

Definition 2.10. With the previous notation, an irreducible representation $\lambda$ of $\mathfrak{m}$ is called fine (with respect to $\mathfrak{g}$ ) if

$$
\left.\lambda\right|_{\mathfrak{m}^{s}} \equiv 0 .
$$

Definition 2.11. A representation $\left(\delta, V_{\delta}\right) \in \widehat{M}$ is called fine (with respect to $G$ ) if under the induced representation of $\mathfrak{m}, V_{\delta}$ is a sum of $\mathfrak{m}$-fine representations (2.10).

Definition 2.12. In the notation (2.9), a representation $\gamma$ of $\mathfrak{k}$ is called fine if 
(a) for the class $[\alpha] \in \Delta / \Gamma$, of $\alpha$ complex, $\left.\gamma\right|_{\mathfrak{t}_{\alpha}} \equiv 0$, and

(b) for $[\alpha] \in \Delta / \Gamma$, of $\alpha$ real, the eigenvalues of $\gamma\left(i Z_{\alpha}\right)$ lie in the range $[-1,1]$.

Definition 2.13. A representation $\left(\mu, V_{\mu}\right) \in \widehat{K}$ is called fine if under the induced representation of $\mathfrak{k}, V_{\mu}$ is a sum of $\mathfrak{k}$-fine representations (2.12).

We want to make use of some results from [V-I] that are proved for $G=G_{o}$ (connected). Therefore, it is important to note:

Remark 2.14 ((Notation (1.2) and Definitions (2.10), (2.11), (2.12) and (2.13)). For $D=M$ or $D=K$, write $D^{o}=D \cap G_{o}$. Let $\gamma$ be in $\widehat{D}$. The following three conditions are equivalent:

(a) $\gamma$ is a $D$-fine representation.

(b) $\left.\gamma\right|_{D^{o}}$ is a sum of $D^{o}$-irreducible fine representations.

(c) $\left.\gamma\right|_{D^{o}}$ contains a $D^{o}$-fine representation.

(The proof is easy; for $D=M$ is trivial, and for $D=K$ we use the fact that $K=M K_{o}$ and $M=T$.)

(2.15) We will be often in the following situation:

Let $A$ and $B$ be a compact Lie groups with $B$ a normal subgroup of $A$. Assume that $R=A / B$ is a finite group. For these pair of groups, there is a homomorphism of groups $c: A \rightarrow \operatorname{Aut}(B)$ defined by $\mathrm{c}(a)(b)=a b a^{-1}$ with $a \in A, b \in B$. We can induce an action of $A$ on $\widehat{B}$ defining $x \cdot \gamma=\gamma\left(c\left(x^{-1}\right)\right)$ for $x \in A$, and $\gamma \in \widehat{B}$. Since under this action $B$ acts trivially on $\widehat{B}$, the action of $A$ factors to an action of $R$ on $\widehat{B}$. If we assume that $R$ is abelian, we make $\widehat{R}$ act on $\widehat{A}$ by tensor product, i.e., given $\gamma \in \hat{A}$ and $\chi \in \widehat{R}$, we define $\chi \cdot \gamma=\chi \otimes \gamma$; then $\chi \cdot \gamma \in \hat{A}$.

(2.16) We add to our notation the following list: Suppose $\delta \in \widehat{B}$ and $\mu \in \widehat{A}$ are such that $\delta$ occurs in $\left.\mu\right|_{B}$; put

$$
\begin{aligned}
A_{\delta} & =\text { stabilizer of } \delta \text { in } A \\
R_{\delta} & =A_{\delta} / B=\text { stabilizer of } \delta \text { in } R \\
\widehat{R}_{\mu} & =\text { stabilizer of } \mu \text { in } \widehat{R} \\
\mu_{\delta} & =\delta \text {-primary subrepresentation of } B \text { on }\left.\mu\right|_{B} .
\end{aligned}
$$

(2.17) Assume we are in situation (2.15) and that $\delta \in \widehat{B}$ occurs in $\mu \in \widehat{A}$ restricted to $B$. The following are well-known facts (see for example [C-R]).

(a) $\mu_{\delta}$ is an irreducible $A_{\delta}$-representation, and $A_{\delta}$ is the stabilizer of $\mu_{\delta}$ in $A, A_{\mu_{\delta}}$.

(b) $\left.\mu\right|_{A_{\delta}}$ is the direct sum decomposition $x_{1} \cdot \mu_{\delta}+x_{2} \cdot \mu_{\delta}+\cdots+x_{r} \cdot \mu_{\delta}$, where $\left\{x_{1}, \cdots, x_{r}\right\}=A / A_{\delta} \quad\left(r=\left|A / A_{\delta}\right|\right)$, and $\delta$ occurs in $\left.x_{j} \cdot \mu_{\delta}\right|_{B}$ if and only if $x_{i} \in A_{\delta}$.

(c) With the usual induction functor for compact groups, we obtain $\mu=$ $\operatorname{Ind}_{A_{\delta}}^{A}\left(\mu_{\delta}\right)$.

From time to time we will write a representation as a pair $(\gamma, V)$ or $\left(\gamma, V_{\gamma}\right)$ to make explicit the underlying complex vector space. 
Definition 2.18. Assume $\delta \in \widehat{M}$ is a fine representation. The set of good roots for $\delta$, denoted $\bar{\Delta}_{\delta}$, consists of roots $\alpha$ in $\bar{\Delta}$ such that, in terms of (2.4), either

(a) $\alpha$ is real and $\delta\left(m_{\alpha}\right) \neq-I$, or

(b) $\alpha$ is complex.

Remark 2.19. For a given real root $\alpha$, and a fine representation $\left(\delta, V_{\delta}\right) \in \widehat{M}$, $\delta\left(m_{\alpha}\right) \neq-I$ is equivalent to the weaker condition that $\delta\left(m_{\alpha}\right)$ does not have $(-1)$ as an eigenvalue.

Proof. Suppose that $\delta\left(m_{\alpha}\right)$ does have $(-1)$ as an eigenvalue. Let $M^{o}=G_{o} \cap$ $M$. Now, restricting $\delta$,

$$
\left.\delta\right|_{M^{o}}=\delta_{o}+m_{1} \cdot \delta_{o}+\cdots+m_{s} \cdot \delta_{o}
$$

for some elements $m_{1}, \cdots, m_{s}$ in $M$, and for some $M^{o}$-irreducible fine representations $\delta_{o}, m_{1} \cdot \delta_{o}, \cdots, m_{s} \cdot \delta_{o}$, by Remark (2.14).

Thus decompose $V_{\delta}$ as follows: $V_{\delta}=V_{\delta_{o}}+V_{m_{1}, \delta_{o}}+\cdots+V_{m_{s}, \delta_{o}}$. We may assume that $(-1)$ is an eigenvalue for $\delta_{o}\left(m_{\alpha}\right)$. Let $v \in V_{\delta_{o}}$ such that $\delta_{o}\left(m_{\alpha}\right) v=-v$. This implies for $j=1,2, \cdots, s$

$$
\begin{aligned}
m_{j} \delta_{o}\left(m_{\alpha}\right)\left(\delta\left(m_{j}\right)\right) v & =\delta_{o}\left(m_{j} m_{\alpha} m_{j}^{-1}\right)\left(\delta\left(m_{j}\right)\right) v=\delta\left(m_{j}\right)\left(\delta_{o}\left(m_{\alpha}\right)\right) v \\
& =-\left(\delta\left(m_{j}\right)\right) v
\end{aligned}
$$

Therefore, for all $j,(-1)$ is also an eigenvalue for $m_{j} \delta_{o}\left(m_{\alpha}\right)$. It is enough to prove the observation for $G=G_{o}$. This is the case proved in [V-I].

Remark 2.20. Taking $\delta$ and $\delta_{o}$ from the proof of Remark (2.16), we conclude $\bar{\Delta}_{\delta}=\bar{\Delta}_{\delta_{o}}$. In [V-I], Vogan proves that $\bar{\Delta}_{\delta_{o}}$, the good roots for $\delta_{o}$, is a root system.

The following property of $K$-representations will allow us to determine them in terms of the Weyl group $W=M^{\prime} / M$.

Proposition 2.21. Suppose we have two $K$-representations $(\mu, V)$ and $\left(\mu^{\prime}, V^{\prime}\right)$ that are fine, (Definition (2.13)). Then

$$
\operatorname{Hom}_{K}\left(\mu, \mu^{\prime}\right)=\operatorname{Hom}_{M^{\prime}}\left(\mu, \mu^{\prime}\right)
$$

Proof. $\subseteq$ is obvious. For the other direction, suppose $T: V \rightarrow V^{\prime}$ is a $M^{\prime}$ homomorphism. To prove that $T$ is a $K$-homomorphism, we use the fact that $K=M^{\prime} K_{o}=M K_{o}$. Thus, it is enough to prove that $T$ is a $K_{o}$-homomorphism or, equivalently, a $\mathfrak{k}$-homomorphism. Recall the decomposition of $\mathfrak{k}$ in (2.9) with respect to $\Delta / \Gamma$. Now, write $\bar{\mu}$ for the induced representation of $\mathfrak{k}$ from $\mu$. For any complex root $\alpha, \bar{\mu}(x)$ and $\overline{\mu^{\prime}}(x)$ are zero for any $x \in \mathfrak{k}_{\alpha}$ (Definition (2.12)). It follows that for any $v \in V$

$$
T(\bar{\mu}(x) v)=T(0)=0=\overline{\mu^{\prime}}(x) T(v) .
$$

Fix $\alpha \in \bar{\Delta}$ a real root. Let us consider a basis $D=\left\{v_{1}, v_{2}, \cdots, v_{m}\right\}$ (respectively $\left.D^{\prime}=\left\{v_{1}^{\prime}, v_{2}^{\prime}, \cdots, v_{m^{\prime}}^{\prime}\right\}\right)$ for $V$ (respectively for $V^{\prime}$ ) such that $v_{j}$ (respectively $v_{j^{\prime}}^{\prime}$ ) is an eigenvector of $\bar{\mu}\left(i Z_{\alpha}\right)$ (respectively of $\overline{\mu^{\prime}}\left(i Z_{\alpha}\right)$ ) with eigenvalue $s_{j}$ (respectively $s_{j^{\prime}}^{\prime}$ ) in $[-1,1]$ for any $j=1,2, \cdots, m$ (respectively $\left.j^{\prime}=1,2, \cdots, m^{\prime}\right)$. Hence, Definition (2.7) implies that for any $j=1,2, \cdots, m$,

$$
\mu\left(\sigma_{\alpha}\right) v_{j}=\mu\left(\exp \left(\frac{\pi}{2} Z_{\alpha}\right)\right) v_{j}=e^{-\frac{\pi}{2} i s_{j}} v_{j}
$$


(and similarly $\mu^{\prime}\left(\sigma_{\alpha}\right) v_{j^{\prime}}=e^{-\frac{\pi}{2} i s_{j^{\prime}}} v_{j^{\prime}}^{\prime}$ for any $j^{\prime}=1,2, \cdots, m^{\prime}$ ). So $\mu\left(\sigma_{\alpha}\right)$ and $\bar{\mu}\left(i Z_{\alpha}\right)$ are simultaneously diagonalizable. For a fixed index $j$, we can write

$T\left(v_{j}\right)=a_{1} \cdot v_{1}^{\prime}+a_{2} \cdot v_{2}^{\prime}+\cdots+a_{m^{\prime}} \cdot v_{m^{\prime}}^{\prime}$ where $a_{k} \in \mathbb{C}$ for each $k=1,2, \cdots, m^{\prime}$. Applying $\mu^{\prime}\left(\sigma_{\alpha}\right)$ to both sides of the equality, we have

$$
a_{1} e^{-\frac{\pi}{2} i s_{1}^{\prime}} \cdot v_{1}^{\prime}+\cdots+a_{m^{\prime}} e^{-\frac{\pi}{2} i s_{m^{\prime}}^{\prime}} \cdot v_{m^{\prime}}^{\prime}=\mu^{\prime}\left(\sigma_{\alpha}\right) T\left(v_{j}\right)=T\left(\mu\left(\sigma_{\alpha}\right) v_{j}\right)=e^{-\frac{\pi}{2} i s_{j}} T\left(v_{j}\right)
$$

where the second equality is given by the invariance of $T$. This implies that for any $a_{k} \neq 0, s_{j}=s_{k}^{\prime}$; because $s_{j}$ and $s_{k}^{\prime}$ are in $[-1,1]$. Therefore,

$$
\overline{\mu^{\prime}}\left(i Z_{\alpha}\right) T\left(v_{j}\right)=s_{j} T\left(v_{j}\right)=T\left(s_{j} v_{j}\right)=T\left(\bar{\mu}\left(i Z_{\alpha}\right) v_{j}\right)
$$

for any $j=1,2, \cdots, m$. This proves the proposition.

Corollary 2.22. Let $(\mu, V)$ and $\left(\mu^{\prime}, V^{\prime}\right)$ be two $K$-fine representations (2.13). Then

(a) The restriction $\left.\mu\right|_{M^{\prime}}$ is irreducible.

(b) $\mu \cong \mu^{\prime}$ if and only if $\left.\left.\mu\right|_{M^{\prime}} \cong \mu^{\prime}\right|_{M^{\prime}}$.

Proof. This a direct consequence of Proposition (2.21).

Example 2.23. We can get to the situation (2.15) for fine representations. Put $A=M^{\prime}, B=M$ and $R=W=M^{\prime} / M$, and suppose $\mu \in \hat{A}$ is the restriction of a $K$-fine irreducible representation (Corollary (2.22)). In the notation (2.16), the result in (2.17) implies

(a) $W_{\mu_{\delta}}=W_{\delta}$ and $M_{\mu_{\delta}}^{\prime}=M_{\delta}^{\prime}$.

(b) $\left.\mu\right|_{M_{\delta}^{\prime}}=w_{1} \cdot \mu_{\delta}+w_{2} \cdot \mu_{\delta}+\cdots+w_{r} \cdot \mu_{\delta}$, for $\left\{w_{1}, \cdots, w_{r}\right\}=M^{\prime} / M_{\delta}^{\prime} \quad(r=$ $\left.\left|M^{\prime} / M_{\delta}^{\prime}\right|\right)$.

(c) $w_{i} \cdot \mu_{\delta} \cong w_{j} \cdot \mu_{\delta}$ if and only if $w_{i}=w_{j}$.

(d) $\mu=\operatorname{Ind}_{M_{\delta}^{\prime}}^{M^{\prime}}\left(\mu_{\delta}\right)$.

(e) $m(\delta, \mu) \stackrel{\delta}{=} m\left(\delta, \mu_{\delta}\right)=m\left(w \cdot \delta, w \cdot \mu_{\delta}\right)=m(w \cdot \delta, \mu)$, for every $w \in$ $W=M^{\prime} / M$.

Definition 2.24. In view of Corollary (2.22), for any fine representation $\delta \in \widehat{M}$, we define

(a) $A(\delta)=\left\{\mu \in \widehat{K} \mid \mu\right.$ is a $K$-fine representation, and $\delta$ occurs in $\left.\left.\mu\right|_{M}\right\}$.

(b) $A_{m}(\delta)=\left\{\mu \in \widehat{M^{\prime}} \mid \mu\right.$ is the restriction of an element in $\left.A(\delta)\right\}$.

(c) $A_{d}(\delta)=\left\{\mu_{\delta} \in \widehat{M_{\delta}^{\prime}} \mid \mu_{\delta}\right.$ is the $\delta$-primary part of an element in $\left.A(\delta)\right\}$.

Remark 2.25. The restriction maps from $A(\delta)$ to $A_{m}(\delta)$ and from $A_{m}(\delta)$ to $A_{d}(\delta)$, Definition (2.24), are bijective.

Proof. Corollary (2.22) implies that the first restriction map is, in fact, bijective. For the second restriction map we find that its inverse is given in $(2.23)(\mathrm{d})$; this is given by the induction functor.

It turns out that $A(\delta) \neq \varnothing$ (Proposition 4.2.3); hence, the other two sets $A_{m}(\delta)$ and $A_{d}(\delta)$ are also non-empty.

(2.26) Recall $\delta, \delta_{o}$, and $\bar{\Delta}_{\delta}=\bar{\Delta}_{\delta_{o}}$ from Remarks (2.19) and (2.20). Denote $W\left(\bar{\Delta}_{\delta}\right)$ for the corresponding Weyl group of $\bar{\Delta}_{\delta}$. In the proof of Proposition (2.21) we saw that for any real root $\alpha \in \bar{\Delta}$, and for any fine representation 
$(\mu, V) \in \widehat{K}$, the eigenvalues of $\mu\left(\sigma_{\alpha}\right)$ are of the form $e^{\frac{\pi}{2} i s}$ with $s \in[-1,1]$. By definition of $\bar{\Delta}_{\delta}$, if the real root $\alpha$ is in $\bar{\Delta}_{\delta}$ then $\delta\left(m_{\alpha}\right) \neq-I$. Then for any $\mu_{\delta} \in A_{d}(\delta)$ (Definition 2.24), $\mu_{\delta}\left(m_{\alpha}\right)$ has eigenvalues of the form $e^{\pi i s}$ with $s \in(-1,1)$. Therefore, we can compute $\mu_{\delta}\left(m_{\alpha}\right)^{\frac{1}{2}}$. This implies that $\mu_{\delta}\left(\sigma_{\alpha}\right)$, being equal to $\mu_{\delta}\left(m_{\alpha}\right)^{\frac{1}{2}}$, can be computed from $\mu_{\delta}\left(m_{\alpha}\right)$; therefore, it leaves invariant each irreducible submodule of $M$ in $V$.

Let $\left(\delta, V_{o}\right) \in \widehat{M}$ be a subrepresentation of $\left.(\mu, V)\right|_{M}$ with $V_{o} \subseteq V$. Fix a real root $\alpha \in \bar{\Delta}_{\delta}$. Thus, we have that for any $m \in M$ and for any $v \in V_{o}$

$$
\sigma_{\alpha} \cdot \delta(m) v=\delta\left(\sigma_{\alpha} m \sigma_{\alpha}^{-1}\right) v=\delta\left(m_{\alpha}\right)^{\frac{1}{2}} \delta(m) \delta\left(m_{\alpha}\right)^{\frac{-1}{2}} v .
$$

Therefore, $\sigma_{\alpha} \cdot \delta$ and $\delta$ are in the same class in $\widehat{M}$. Since $\sigma_{\alpha} \cdot \delta$ is in the class $s_{\alpha} \cdot \delta, s_{\alpha} \in W\left(\bar{\Delta}_{\delta}\right)$. So, the conclusion is $s_{\alpha} \cdot \delta=\delta$. On the other hand, for any complex root $\beta \in \bar{\Delta}_{\delta}, s_{\beta} \in W\left(\bar{\Delta}_{\delta}\right)$ has a representative $k=\exp (x) \in K$ with $x \in \sum_{[\alpha] \in \Delta / \Gamma,\left.\alpha\right|_{a}=\beta}\left(\mathfrak{k}_{\alpha}\right)$ (see decomposition of $\mathfrak{k}$ in (2.9)).

Thus, we have that $k \cdot \delta$ is a representative for $s_{\beta} \cdot \delta$ in $\widehat{M}$ and $\mu_{\delta}(k)$ is the identity by definition of being fine.

For any $v \in V$, and for any $m \in M$

$$
k \cdot \delta(m) v=\delta\left(k m k^{-1}\right) v=\mu_{\delta}(k) \delta(m) \mu_{\delta}\left(k^{-1}\right) v
$$

This implies that $k \cdot \delta$ and $\delta$ are representatives of the same class in $\widehat{M}$. In other words, $s_{\beta} \cdot \delta=\delta$; identifying $\delta$ with its own class. We conclude that $W\left(\bar{\Delta}_{\delta}\right) \subseteq W_{\delta}$.

In fact, more is true.

Proposition 2.27. Assume we are in the setting (2.26). Under the Weyl group action, $W_{\delta}$ normalizes $\bar{\Delta}_{\delta}$. In particular, $W\left(\bar{\Delta}_{\delta}\right)$ is a normal subgroup of $W_{\delta}$. Proof. The second statement follows from the first one. To prove the first assertion is enough to assume that $G=G_{o}$. This case is proved in [V-I].

Definition 2.28. We denote,

$$
\begin{aligned}
\left(M_{\delta}^{\prime}\right)^{o} & =\text { inverse image of } W\left(\bar{\Delta}_{\delta}\right) \text { in } M^{\prime}, \\
W_{\delta}^{o} & =\left(M_{\delta}^{\prime}\right)^{o} / M=W\left(\bar{\Delta}_{\delta}\right), \\
R_{\delta} & =W_{\delta} / W_{\delta}^{o}=M_{\delta}^{\prime} /\left(M_{\delta}^{\prime}\right)^{o} .
\end{aligned}
$$

$R_{\delta}$ is called the $R$-group associated to $\delta$.

(2.29) Now, let us fix a system of positive roots $\bar{\Delta}_{\delta}^{+}$for $\bar{\Delta}_{\delta}=\bar{\Delta}_{\delta_{o}}$ and define (Notation (1.2))

$$
\rho_{\delta_{o}}=\rho_{\delta}=\rho\left(\bar{\Delta}_{\delta}^{+}\right) .
$$

Choose a positive root system $\bar{\Delta}^{+}$for $\bar{\Delta}$ such that $\rho_{\delta_{o}}$ is dominant. Then define

$$
\bar{\Delta}_{s}=\left\{\alpha \in \bar{\Delta} \mid\left\langle\alpha, \rho_{\delta_{o}}\right\rangle=0\right\}
$$

and $\bar{\Delta}_{s}^{+}=\bar{\Delta}_{s} \cap \bar{\Delta}^{+}$.

In [V-I], we find important properties involving $\bar{\Delta}_{s}$. 
Proposition 2.30. In the notation above, the following holds:

(a) $\bar{\Delta}_{s}$ is a root system, $\bar{\Delta}_{s}^{+}$is a strongly orthogonal set $\left\{\alpha_{1}, \cdots, \alpha_{r}\right\}$ of simple roots of $\bar{\Delta}^{+}$.

(b) If $\alpha \in \bar{\Delta}_{s}, \alpha$ is real and $\delta_{o}\left(m_{\alpha}\right)=-I$.

(Using Remark (2.19), $\alpha \in \bar{\Delta}_{s}$ implies also that $\delta\left(m_{\alpha}\right)=-I$.)

Definition 2.31. Write $R_{\delta}^{c}=\left\{w \in W_{\delta} \mid w\left(\bar{\Delta}_{\delta}^{+}\right)=\bar{\Delta}_{\delta}^{+}\right\}$.

By definition, $R_{\delta}^{c}$ is the stabilizer of $\bar{\Delta}_{\delta}^{+} \subseteq \bar{\Delta}_{\delta}$ in $W_{\delta}$ (Proposition (2.27)). Write $W\left(\bar{\Delta}_{s}\right)$ for the Weyl group $\bar{\Delta}_{s}$. It is clear that $W\left(\bar{\Delta}_{s}\right) \cong(\mathbb{Z} / 2 \mathbb{Z})^{r}$.

Proposition 2.32 (Definition (2.28)). $W_{\delta}$ can be decomposed as follows:

(a) $W_{\delta}=R_{\delta}^{c} W_{\delta}^{o}$.

(b) $R_{\delta}^{c} \subseteq W\left(\bar{\Delta}_{s}\right)$.

Proof. The proposition uses only the properties of the group of automorphisms of a root system. For (b) we apply Chevalley's theorem ([Green], Lemma (4.3.29)).

Corollary 2.33 (Definition (2.28)). The $R$-group $R_{\delta}$ is a direct sum of copies of the group $\mathbb{Z} / 2 \mathbb{Z}$.

\section{THE $R$-GROUP}

Vogan constructs an action of $\widehat{R_{\delta}}$ on $A(\delta)$ (Definition (2.24) (a)) by using certain involutive automorphisms of the maximal compact subgroup $K$; they can be thought as extensions for the characters of $R_{\delta}$ ([Green], Definition 4.3.47). These extensions do not exist when our group $G$ is non-linear. However, we can still build up an action of $\widehat{R_{\delta}}$ on $A_{d}(\delta)$. As consequence of Remark (2.25), we extend this action on $A_{d}(\delta)$ to one on $A(\delta)$.

(3.1) Let $\delta$ be a fine representation of $M$. Consider $A_{d}(\delta)$ as in Definition (2.24)(c); $A_{d}(\delta) \subseteq \widehat{M_{\delta}^{\prime}}$. Let $R_{\delta}$ be the $R$-group associated to $\delta$. In the situation (2.15), $\widehat{R_{\delta}}$ acts on $\widehat{M_{\delta}^{\prime}}$. Let $\mu_{\delta} \in A_{d}(\delta)$ and write $\widehat{R_{\delta}} \cdot \mu_{\delta}$ for the orbit of $\mu_{\delta}$ in $\widehat{M_{\delta}^{\prime}}$ under the action of $\widehat{R_{\delta}}$. We want to prove that $A_{d}(\delta)=\widehat{R_{\delta}} \cdot \mu_{\delta}$. The next result provides half of the desired statement.

Proposition 3.2. $A_{d}(\delta) \subseteq \widehat{R_{\delta}} \cdot \mu_{\delta}$.

Proof. Let $\left(\mu_{\delta}, V_{\mu_{\delta}}\right)$ and $\left(\mu_{\delta}^{\prime}, V_{\mu_{\delta}^{\prime}}^{\prime}\right)$ be in $A_{d}(\delta)$ which are restrictions of $(\mu, V)$ and $\left(\mu^{\prime}, V^{\prime}\right)$ in $A(\delta)$, respectively Remark (2.25). Define $V_{\mu, \mu^{\prime}}=$ $\operatorname{Hom}_{M}\left(V_{\mu_{\delta}}, V_{\mu_{\delta}^{\prime}}^{\prime}\right)$.

(a) $V_{\mu, \mu^{\prime}}$ is a $M_{\delta}^{\prime}$-module as follows : For each homomorphism $T \in V_{\mu, \mu^{\prime}}$, and each vector $v \in V_{\delta}$, define

$$
(x \cdot T)(v)=\mu_{\delta}^{\prime}(x) T\left(\mu_{\delta}\left(x^{-1}\right) v\right)=x\left(T\left(x^{-1} v\right)\right) .
$$

Since $M$ is a normal subgroup of $M_{\delta}^{\prime}$, the action of $M_{\delta}^{\prime}$ on $V_{\mu, \mu^{\prime}}$ is welldefined.

(b) The action in (a) restricted to the group $\left(M_{\delta}^{\prime}\right)^{o}$, defined in $(2.28)$, is trivial. To see this, we note that $\left(M_{\delta}^{\prime}\right)^{o}$ is generated by $M$ and $\left\{\sigma_{\alpha}\right\}_{\alpha \in \bar{\Delta}_{\delta}}$. For a complex root $\beta \in \bar{\Delta}_{\delta}^{+}, \sigma_{\beta}$ fixes any element of $V_{\mu, \mu^{\prime}}$. This is so because, 
by definition, $\mu_{\delta}\left(\sigma_{\beta}\right)$ and $\mu_{\delta}^{\prime}\left(\sigma_{\beta}\right)$ are the identity maps. On the other hand, for any real root $\alpha \in \bar{\Delta}_{\delta}^{+}$, we observed in (2.26) that $\mu_{\delta}\left(\sigma_{\alpha}\right)$ and $\mu_{\delta}^{\prime}\left(\sigma_{\alpha}\right)$ can be computed as $\mu_{\delta}\left(m_{\alpha}\right)^{\frac{1}{2}}$ and $\mu_{\delta}^{\prime}\left(m_{\alpha}\right)^{\frac{1}{2}}$ respectively. Therefore, $\bar{\mu}\left(i Z_{\alpha}\right)$ leaves invariant $V_{\mu_{\delta}}$ (resp. $\overline{\mu^{\prime}}\left(i Z_{\alpha}\right)$ on $\left.V_{\mu_{\delta}^{\prime}}^{\prime}\right)$ (Definition (2.5)). In the same fashion as in the proof of Proposition (2.21) let us consider a basis $D=\left\{v_{1}, v_{2}, \cdots, v_{m}\right\}$ (resp. $D^{\prime}=\left\{v_{1}^{\prime}, v_{2}^{\prime}, \cdots, v_{m^{\prime}}^{\prime}\right\}$ ) for $V_{\mu_{\delta}}$ (resp. for $V_{\mu_{\delta}^{\prime}}^{\prime}$ ) such that $v_{j}$ (resp. $\left.v_{j^{\prime}}^{\prime}\right)$ is an eigenvector of $\bar{\mu}\left(i Z_{\alpha}\right)$ (resp. of $\left.\overline{\mu^{\prime}}\left(i Z_{\alpha}\right)\right)$ with eigenvalue $s_{j}$ (resp. $\left.s_{j^{\prime}}^{\prime}\right)$ in $(-1,1)$ for any $j=1,2, \cdots, m$ (resp. $\left.j^{\prime}=1,2, \cdots, m^{\prime}\right)$. Hence, Definition (2.7) implies for any $j=1,2, \cdots, m$,

$$
\mu_{\delta}\left(\sigma_{\alpha}\right) v_{j}=\mu_{\delta}\left(\exp \left(\frac{\pi}{2} Z_{\alpha}\right)\right) v_{j}=e^{-\frac{\pi}{2} i s_{j}} v_{j}
$$

(and similarly $\mu_{\delta}^{\prime}\left(\sigma_{\alpha}\right) v_{j^{\prime}}=e^{-\frac{\pi}{2} i s_{j^{\prime}}} v_{j^{\prime}}^{\prime}$ for any $j^{\prime}=1,2, \cdots, m^{\prime}$ ). Now, let $T$ be in $V_{\mu, \mu^{\prime}}$. Fix an index $j$. Suppose, $T\left(v_{j}\right)=a_{1} \cdot v_{1}^{\prime}+a_{2} \cdot v_{2}^{\prime}+\cdots+a_{m^{\prime}} \cdot v_{m^{\prime}}^{\prime}$ where $a_{k} \in \mathbb{C}$ for each $k=1,2, \cdots, m^{\prime}$. Applying $\mu_{\delta}^{\prime}\left(m_{\alpha}\right)$ to both sides of this last equality, we have

$$
\begin{aligned}
a_{1} e^{-\pi i s_{1}^{\prime}} \cdot v_{1}^{\prime}+\cdots+a_{m^{\prime}} e^{-\pi i s_{m^{\prime}}^{\prime} \cdot v_{m^{\prime}}^{\prime}} & =\mu_{\delta}^{\prime}\left(m_{\alpha}\right) T\left(v_{j}\right)=T\left(\mu_{\delta}\left(m_{\alpha}\right) v_{j}\right) \\
& =e^{-\pi i s_{j}} T\left(v_{j}\right) .
\end{aligned}
$$

Hence, for any $a_{k} \neq 0$ we have $s_{k}^{\prime}=s_{j}$, because $s_{k}^{\prime}$ and $s_{j}$ are in the interval $(-1,1)$. Therefore, for any $j=1,2, \cdots, m$,

$$
\mu_{\delta}^{\prime}\left(\sigma_{\alpha}\right) T\left(v_{j}\right)=e^{-\frac{\pi}{2} i s_{j}} T\left(v_{j}\right)=T\left(e^{-\frac{\pi}{2} i s_{j}} \cdot v_{j}\right)=T\left(\mu_{\delta}\left(\sigma_{\alpha}\right) v_{j}\right)
$$

The assertion of statement $(b)$ follows.

(c) By statement (b), the action of $M_{\delta}^{\prime}$ on $V_{\mu, \mu^{\prime}}$ factors to an action of $R_{\delta}$, (Definition (2.28)). Define a homomorphism of $M_{\delta}^{\prime}$-modules $\varphi: V_{\mu, \mu^{\prime}} \otimes \mu_{\delta} \rightarrow$ $\mu_{\delta}^{\prime}$ by $\varphi(F \otimes v)=F(v)$ for $F \in V_{\mu, \mu^{\prime}}$, and $v \in V_{\mu_{\delta}}$. For each $m \in M_{\delta}^{\prime}$,

$$
\begin{aligned}
m(\varphi(F \otimes v)) & =m(F(v))=m\left(F\left(m^{-1}(m v)\right)\right)=(m \cdot F)(m v) \\
& =\varphi(m \cdot F \otimes m v)=\varphi(m(F \otimes v))
\end{aligned}
$$

On the other hand, $V_{\mu, \mu^{\prime}} \neq 0$ and $\varphi \not \equiv 0$, for $\mu_{\delta}, \mu_{\delta}^{\prime} \in A_{d}(\delta)$. Since $R_{\delta}$ is abelian by Corollary (2.33), $V_{\mu, \mu^{\prime}}$ can be decomposed into a direct sum of one-dimensional representations of $R_{\delta}$. Therefore, there exists $\chi \in \widehat{R_{\delta}}$ such that $\varphi \mid: \chi \otimes \mu_{\delta} \rightarrow \mu_{\delta}^{\prime}$ is a non-zero isomorphism of $M_{\delta}^{\prime}$-modules. Thus $\mu_{\delta}^{\prime}=\chi \cdot \mu_{\delta} \in \widehat{R_{\delta}} \cdot \mu_{\delta}$. Hence, Proposition (3.2) holds.

Corollary 3.3. For any pair $\mu$ and $\mu^{\prime}$ in $A(\delta)$, Definition (2.24), we have

(a) $m(\delta, \mu)=m\left(\delta, \mu_{\delta}\right)=m\left(\delta, \mu_{\delta}^{\prime}\right)=m\left(\delta, \mu^{\prime}\right)$.

(b) $m(\delta, \mu)=\sum_{\gamma \in\left(\widehat{\left.M_{\delta}^{\prime}\right)^{0}}\right.} \operatorname{dim}\left(\operatorname{Hom}_{\left(M_{\delta}^{\prime}\right)}\left(\gamma, \mu_{\delta}\right)\right)$.

Proof. (a) is a direct consequence of Proposition (3.2). Meanwhile, (b) holds because of (b) in the proof of Proposition (3.2): $V_{\mu, \mu^{\prime}} \cong \operatorname{Hom}_{\left(M_{\delta}^{\prime}\right)}\left(\mu_{\delta}, \mu_{\delta}^{\prime}\right)$.

Definition 3.4. Suppose $\mu_{\delta} \in A_{d}(\delta)$. Set

$$
\widehat{R_{\delta}^{o}}=\left\{\chi \in \widehat{R_{\delta}} \mid \chi \otimes \mu_{\delta}=\mu_{\delta}\right\} \text { and } \widehat{R_{\delta}^{1}}=\widehat{R_{\delta}} / \widehat{R_{\delta}^{o}} .
$$

It is clear that our Definition (3.4) is independent of $\mu_{\delta}$. 
Proposition 3.5. In the notation of (3.4), we have:

(a) For any $\mu_{\delta} \in A_{d}(\delta)$, we have $A_{d}(\delta)=\widehat{R_{\delta}} \cdot \mu_{\delta}=\widehat{R_{\delta}^{1}} \cdot \mu_{\delta}$.

(b) For any $\mu_{\delta} \in A_{d}(\delta)$, the multiplicity of $\delta$ in $\left.\mu_{\delta}\right|_{M}$ is $m\left(\delta, \mu_{\delta}\right)=\left|\widehat{R_{\delta}^{o}}\right|^{\frac{1}{2}}$.

Statement (a) of Proposition (3.5) means that we have an action of $\widehat{R_{\delta}}$ on $A_{d}(\delta)$ that is transitive.

The proof of Proposition (3.5) will be given in two parts. Proposition (3.5) (a) is proved in Lemma (4.3.3) and Proposition (3.5) (b) is Lemma (3.8) (b).

(3.6) As preparation for the proof of Proposition (3.5), we formulate three lemmas.

For the rest of this section, assume we are in the situation (2.15). Suppose $R=A / B$ is an abelian group, $\delta \in \widehat{B}, \mu \in \widehat{A}$ and $\delta$ occurs in $\left.\mu\right|_{B}$. Recall the definitions for $A_{\delta}, R_{\delta}, \widehat{R}_{\mu}$ and $\mu_{\delta}$ from there. Write also $N=A / A_{\delta}$.

Lemma 3.7. Denote $\left(\widehat{R_{\delta}}\right)_{\mu_{\delta}}$ for the stabilizer of $\mu_{\delta}$ in $\widehat{R_{\delta}}$. We have two short exact sequences

$$
\begin{gathered}
1 \rightarrow \widehat{N} \rightarrow \widehat{R} \stackrel{\Psi}{\longrightarrow} \widehat{R_{\delta}} \rightarrow 1, \\
1 \rightarrow \widehat{N} \rightarrow \widehat{R}_{\mu} \stackrel{\Psi}{\longrightarrow}\left(\widehat{R_{\delta}}\right)_{\mu_{\delta}} \rightarrow 1
\end{gathered}
$$

where $\Psi(\chi)=\left.\chi\right|_{A_{\delta}}$

Proof. $1 \rightarrow R_{\delta} \rightarrow R \rightarrow N \rightarrow 1$ is a short exact sequence of abelian groups.

Hence, (a) holds. What (b) claims is that $\chi \cdot \mu=\mu$ if and only if $\left.\chi\right|_{A_{\delta}} \cdot \mu_{\delta}=\mu_{\delta}$. Assume $\chi \otimes \mu=\mu$. It implies that $\left.\chi\right|_{A_{\delta}} \otimes \mu_{\delta}=x \cdot \mu_{\delta}$ for some $x$ element of $A$. But, since $\left.\chi\right|_{A_{\delta}} \otimes \mu_{\delta}$ restricted to $B$ is a sum of $\delta$ 's, then $x \in A_{\delta}$; therefore, $x \cdot \mu_{\delta}=\mu_{\delta}$. To prove the other direction, we make use of the fact (2.17) which shows

$$
\mu=\operatorname{Ind}_{A_{\delta}}^{A}\left(\mu_{\delta}\right)=\operatorname{Ind}_{A_{\delta}}^{A}\left(\left.\chi\right|_{A_{\delta}} \otimes \mu_{\delta}\right)=\chi \otimes \mu
$$

Lemma (3.7) follows.

Lemma 3.8. Suppose again that $R=A / B$ is abelian, situation (3.6). Then,

(a) The restriction $\left.\mu\right|_{B}$ is irreducible if and only if $\widehat{R}_{\mu}=1$.

(b) $m(\delta, \mu)=m\left(\delta, \mu_{\delta}\right)=\left|\left(\widehat{R_{\delta}}\right)_{\mu_{\delta}}\right|^{\frac{1}{2}}$.

(c) $A_{\delta}=B$ if and only if $\widehat{R}_{\mu}=\widehat{R}$ and $m(\delta, \mu)=1$.

Proof. Mackey's theory tell us that $\operatorname{Ind}_{B}^{A}\left(\left.\mu\right|_{B}\right)=\sum_{\chi \in \widehat{R}} \chi \cdot \mu$. Next, we apply the Frobenius reciprocity theorem:

$$
\operatorname{Hom}_{A}\left(\mu, \operatorname{Ind}_{B}^{A}\left(\left.\mu\right|_{B}\right)\right)=\operatorname{Hom}_{B}\left(\left.\mu\right|_{B},\left.\mu\right|_{B}\right)
$$

Therefore, $\operatorname{Hom}_{B}\left(\left.\mu\right|_{B},\left.\mu\right|_{B}\right)=\sum_{\chi \in \hat{R}} \operatorname{Hom}_{A}(\mu, \chi \cdot \mu)=\mathbb{C}^{m}$ where $m=\left|\widehat{R}_{\mu}\right|$. This implies that $\left.\mu\right|_{B}$ is irreducible if and only if $m=1$. This proves (a).

To prove (b) we use result (2.17). We get for $\left\{x_{1}, \cdots, x_{r}\right\}=\left(A / A_{\delta}\right)$,

$$
\mathbb{C}^{m}=\operatorname{Hom}_{B}\left(\left.\mu\right|_{B},\left.\mu\right|_{B}\right)=\sum_{i=1}^{r} \operatorname{Hom}_{B}\left(\left.x_{i} \cdot \mu_{\delta}\right|_{B},\left.x_{i} \cdot \mu_{\delta}\right|_{B}\right)=\sum_{1}^{r} \operatorname{Hom}_{B}\left(\left.\mu_{\delta}\right|_{B},\left.\mu_{\delta}\right|_{B}\right)
$$


where $r=\left|A / A_{\delta}\right|$. Conclude that $m(\delta, \mu)^{2}=\operatorname{dim}\left(\operatorname{Hom}_{B}\left(\left.\mu_{\delta}\right|_{B},\left.\mu_{\delta}\right|_{B}\right)\right)=$ $\left|\widehat{R}_{\mu}\right|\left|A / A_{\delta}\right|^{-1}$. Hence, Lemma (3.7) (b) implies Lemma (3.8) (b). Similarly, (c) is consequence of this. The lemma follows.

From Lemma (3.7) and Lemma (3.8), we get the following consequence.

Corollary 3.9. We obtain

(a) $\widehat{R} / \widehat{R}_{\mu}=\widehat{R_{\delta}} /\left(\widehat{R_{\delta}}\right)_{\mu_{\delta}}$.

(b) $|\widehat{N}|^{-1}\left|\widehat{R}_{\mu}\right|=m(\delta, \mu)^{2}$.

(3.10) Now, suppose we have a third group $C$ such that $B \subseteq C \subseteq A$. Suppose also that $R=A / B$ is an abelian group. Denote $N_{1}=A / C$ and $S=C / B$.

Let us assume that $\eta \in \widehat{C}$ is such that it occurs in $\left.\mu\right|_{C}$ and $\left.\eta\right|_{B}$ contains $\delta$ as submodule. First note that $C \subseteq A_{\eta} \subseteq A_{\delta} C$ : since if $x \cdot \eta=\eta$ for some $x \in A$, this implies that $x \cdot \delta=y \cdot \delta$ for some $y \in C$; that is, $y^{-1} x \in A_{\delta}$ and then $x \in A_{\delta} C$. For $\eta$, we write

$$
\begin{aligned}
& Q=\left\{\chi \in \widehat{R}|\chi|_{C} \otimes \eta=x \cdot \eta \quad \text { for some } x \in A\right\}, \\
& Q_{o}=\left\{\chi \in \widehat{R}|\chi|_{C} \otimes \eta=\eta\right\}
\end{aligned}
$$

Lemma 3.11. We have

(a) $Q / Q_{o} \cong\left(A_{\delta} C / A_{\eta}\right)$.

(b) $m(\delta, \mu)=m(\delta, \eta) m(\eta, \mu)\left|Q / Q_{o}\right|$.

Proof. As in Lemma (3.7) (a), the following is a short exact sequence

$$
1 \rightarrow \widehat{N_{1}} \rightarrow \widehat{R} \stackrel{\Psi}{\longrightarrow} \widehat{S} \rightarrow 1
$$

where $\Psi(\chi)=\left.\chi\right|_{C}$. Clearly $Q_{o}=\Psi^{-1}\left(\widehat{S}_{\eta}\right)$. Thus, $\widehat{R} / Q_{o} \cong \widehat{S} /\left(\widehat{S}_{\eta}\right)$.

We have natural actions of $\widehat{S} \times\left(A_{\delta} C\right)$ on $\widehat{C}$ and $\widehat{R} \times \widehat{N}_{1}$ on $\widehat{A}$.

(1) $\widehat{R} \times \widehat{N_{1}}$ acts on $\widehat{A}$ by tensor product, as $\widehat{R}$ does.

(2) $\widehat{S} \times\left(A_{\delta} C\right)$ acts on $\widehat{C}$ as follows: given $(\chi, y) \in \widehat{S} \times\left(A_{\delta} C\right)$ and $\omega \in \widehat{C}$, we define $(\chi, y) \cdot \omega=y \cdot(\chi \otimes \omega)$. (Since $S$ is abelian, $y \cdot \chi=\chi$. Then this action is well-defined.)

We are interested in the orbit $\Omega=\left(\widehat{S} \times\left(A_{\delta} C\right)\right) \cdot \eta$ contained in $\widehat{C}$. The action of $\widehat{S} \times\left(A_{\delta} C\right)$ on $\Omega$ factors to an action of $\widehat{S} \times\left(A_{\delta} C / A_{\eta}\right)$. Write $\left(\widehat{S} \times\left(A_{\delta} C / A_{\eta}\right)\right)_{\eta}$ for the stabilizer of $\eta$ in $\widehat{S} \times\left(A_{\delta} C / A_{\eta}\right)$ and $\left(\widehat{R} \times \widehat{N_{1}}\right)_{\mu}$ for the stabilizer of $\mu$ in $\widehat{R} \times \widehat{N_{1}}$.

We have three short exact sequences:

$$
\begin{aligned}
1 \rightarrow \widehat{S}_{\eta} & \rightarrow\left(\widehat{S} \times\left(A_{\delta} C / A_{\eta}\right)\right)_{\eta} \stackrel{\pi_{2}}{\longrightarrow} A_{\delta} C / A_{\eta} \rightarrow 1, \\
1 \rightarrow \widehat{N_{1}} & \rightarrow Q \stackrel{\varphi}{\longrightarrow}\left(\widehat{S} \times\left(A_{\delta} C / A_{\eta}\right)\right)_{\eta} \rightarrow 1, \\
1 & \rightarrow \widehat{N}_{1 \mu} \rightarrow\left(\widehat{R} \times \widehat{N_{1}}\right)_{\mu} \stackrel{\pi_{1}}{\longrightarrow} Q \rightarrow 1
\end{aligned}
$$

where $\pi_{i}$ is the projection with respect $i$-factor, $i \in\{1,2\}$, and $\varphi$ is defined in the following way. For any $\chi \in Q$, there exists, by definition, an element $y$ in $A$ such that $\left.\chi\right|_{C} \cdot \eta=y \cdot \eta$. Then define $\varphi(\chi)=\left(\left.\chi\right|_{C}, \bar{y}^{-1}\right)$ where $\bar{y}$ is the class of $y$ in $A / A_{\eta}$. We claim that $y$ is an element of $A_{\delta} C$. But $\left.\left(\left.\chi\right|_{C} \cdot \eta\right)\right|_{B}$ contains $\delta$ as submodule, then $\left.(y \cdot \eta)\right|_{B}$ also contains $\delta$. This implies the 
existence of an element $c \in C$ such that $\delta=c \cdot y \cdot \delta$. Therefore, $y \cdot c \in A_{\delta}$ and then $y \in A_{\delta} C$.

The group $Q_{o}$ is also equal to $\varphi^{-1}\left(\widehat{S}_{\eta}\right)$. Hence $Q / Q_{o} \cong\left(\widehat{S} \times\left(A_{\delta} C / A_{\eta}\right)\right)_{\eta} / \widehat{S}_{\eta}$, and $\left(\widehat{S} \times\left(A_{\delta} C / A_{\eta}\right)\right)_{\eta} / \widehat{S}_{\eta} \cong A_{\delta} C / A_{\eta}$. This proves (a). On the other hand, we have the short exact sequence:

$$
1 \rightarrow \widehat{R}_{\mu} \rightarrow\left(\widehat{R} \times \widehat{N_{1}}\right)_{\mu} \stackrel{\pi_{2}}{\longrightarrow} \widehat{N_{1}} \rightarrow 1
$$

Applying cardinality,

$$
\begin{aligned}
\left|\widehat{R}_{\mu}\right|\left|\widehat{N}_{1}\right| & =\left|\left(\widehat{R} \times \widehat{N}_{1}\right)_{\mu}\right|=\left|\widehat{N}_{1 \mu}\right||Q|=\left|\widehat{N}_{1 \mu}\right|\left|\widehat{N_{1}}\right|\left|\left(\widehat{S} \times\left(A_{\delta} C / A_{\eta}\right)\right)_{\eta}\right| \\
& =\left|\widehat{N}_{1 \mu}\right|\left|\widehat{N}_{1}\right|\left|\widehat{S}_{\eta}\right|\left|A_{\delta} C / A_{\eta}\right|
\end{aligned}
$$

In short, $\left|\widehat{R}_{\mu}\right|=\left|\widehat{N}_{1}\right|\left|\widehat{S}_{\eta} \| A_{\delta} C / A_{\eta}\right|$. This, in turn, implies

$$
\begin{aligned}
\left|A / A_{\delta}\right|^{-1}\left|\widehat{R}_{\mu}\right| & =\left|A / A_{\eta}\right|^{-1}\left|A_{\delta} C / A_{\eta} \| A_{\delta} C / A_{\delta}\right|^{-1}\left|\widehat{R}_{\mu}\right| \\
& =\left|A / A_{\eta}\right|^{-1}\left|\widehat { N } _ { 1 } \left\|C /\left.C_{\delta}\right|^{-1}\left|\widehat{S}_{\eta} \| A_{\delta} C / A_{\eta}\right|^{2}\right.\right.
\end{aligned}
$$

In view of Corollary (3.9) (b), we conclude

$$
m(\delta, \mu)^{2}=m(\delta, \eta)^{2} m(\eta, \mu)^{2}\left|A_{\delta} C / A_{\eta}\right|^{2}
$$

This proves Lemma (3.11).

\section{REAL PARABOLIC INDUCTION AND $(\mathfrak{g}, K)$-MODULES}

4.1 Admissible representations. For a group $G$ in the Harish-Chandra class with maximal compact subgroup $K$, let $\mathfrak{g}$ and $\mathfrak{k}$ be the complexification of $\mathfrak{g}_{o}=\operatorname{Lie}(G)$ and $\mathfrak{k}_{o}=\operatorname{Lie}(K)$ repectively. Write $\mathfrak{U}(\mathfrak{g})$ for the universal enveloping algebra associated to $\mathfrak{g}$.

The concept of admissible representations was introduced by Harish-Chandra on comparing the irreducible unitary representations of a semisimple Lie group with irreducible representations of its Lie algebra. This concept was developed and generalized for groups in the Harish-Chandra class by many people, notably by Lepowsky in his paper [L]. This work gave rise to the definition of the category of $(\mathfrak{g}, K)$-modules.

(4.1.1) We recall that the definition of a representation $(\Pi, \mathfrak{X})$ of $G$ on a complex Hilbert space is given by a continuous homomorphism $\Pi: G \rightarrow \mathfrak{B}(\mathfrak{X})$. We assume that $\mathfrak{B}(\mathfrak{X})$, the semigroup of bounded operators on $\mathfrak{X}$, is endowed with the weak topology, and $\Pi(1)$ is the identity operator. When $\Pi(G)$ consists only of unitary operators, we say $(\Pi, \mathfrak{X})$ is a unitary representation. Now, given two (unitary) representations $(\Pi, \mathfrak{X})$ and $(\Sigma, \mathfrak{W})$ of $G$, we say they are (unitarily) boundedly equivalent if there is an invertible operator $Q: \mathfrak{X} \rightarrow$ $\mathfrak{W}$ such that $Q$ and $Q^{-1}$ are (unitary) bounded intertwining operators w.r.t. $\Pi$ and $\Sigma$.

Definition 4.1.2. A $(\mathfrak{g}, K)$-module is a pair $(\pi, V)$ with $V$ a complex vector space, and $\pi: \mathfrak{U}(\mathfrak{g}) \sqcup K \rightarrow \operatorname{End}(V)$ is a map which, restricted, is simultaneously a $\mathfrak{U}(\mathfrak{g})$-module and a representation of $K$, satisfying

(a) $V$ consists of $K$-finite vectors (i.e., any $v$ in $V$ satisfies $\operatorname{dim}\langle K \cdot v\rangle<$ $\infty)$. 
(b) The differential of the representation of $K$ on $V$ is the same as the representation of $\mathfrak{U}(\mathfrak{g})$ restricted to $\mathfrak{k}_{o}=\operatorname{Lie}(K)$.

(c) If $X \in \mathfrak{g}, \quad k \in K$ and $v \in V$ then

$$
\pi([\operatorname{Ad}(k) X]) \cdot v=\pi(k)\left(\pi(X)\left(\pi\left(k^{-1}\right) \cdot v\right)\right) .
$$

The set of $(\mathfrak{g}, K)$-homomorphisms for two $(\mathfrak{g}, K)$-modules $(\pi, V)$ and $(\sigma, W)$ is defined by the following collection:

$$
\begin{array}{r}
\operatorname{Hom}_{(\mathfrak{g}, K)}(V, W):=\{Q: V \rightarrow W \mid Q \text { is complex and linear, } \\
\text { such that } Q \pi=\sigma Q\} .
\end{array}
$$

We say $(\pi, V)$ and $(\sigma, W)$ are equivalent if there is an invertible element in $\operatorname{Hom}_{(\mathfrak{g}, K)}(V, W)$. The category whose objects are all $(\mathfrak{g}, K)$-modules and the maps are given by all the sets $\operatorname{Hom}_{(\mathfrak{g}, K)}(V, W)$ will be denoted by $\mathfrak{c}(\mathfrak{g}, K)$.

Next, we define the category $\mathfrak{a}(\mathfrak{g}, K)$, of admissible $(\mathfrak{g}, K)$-modules.

Definition 4.1.3. Let $(\pi, V)$ be any object in $\mathfrak{c}(\mathfrak{g}, K)$.

(a) $(\pi, V)$ is called admissible if for any $(\sigma, W) \in \widehat{K}, \operatorname{dim}\left(\operatorname{Hom}_{K}(W, V)\right)$ is finite.

(b) $(\pi, V)$ is called of finite length if it is so as a $\mathfrak{U}(\mathfrak{g})$-module.

(4.1.4) Denote by $\mathfrak{a}(\mathfrak{g}, K)$ the full subcategory of admissible $(\mathfrak{g}, K)$-modules of $\mathfrak{c}(\mathfrak{g}, K)$, and by $\mathfrak{F}(\mathfrak{g}, K)$ the full subcategory of objects of finite length in $\mathfrak{a}(\mathfrak{g}, K)$.

(4.1.5) For a representation $(\Pi, \mathfrak{X})$ of $G$ we denote the set of $K$-finite vectors by $\mathfrak{X}_{K}((4.1 .2)(\mathrm{a}))$. $(\Pi, \mathfrak{X})$ is called admissible if for any $(\sigma, W) \in \widehat{K}$, $\operatorname{dim}\left(\operatorname{Hom}_{K}\left(W, \mathfrak{X}_{K}\right)\right)$ is finite. We construct the Harish-Chandra correspondence:

For any admissible representation $(\Pi, \mathfrak{X})$ of $G$ we associate to it the module $\left(\dot{\pi}, \mathfrak{X}_{K}\right)$ of $(\mathfrak{g}, K)$ on the set of $K$-finite vectors of $\mathfrak{X}$, given by the restrictions $\left.\dot{\pi}\right|_{K}=\left.\Pi\right|_{K}$ and $\left.\dot{\pi}\right|_{\mathfrak{g}_{o}}=\left.d \Pi\right|_{\mathfrak{x}_{K}}$. This correspondence is well-defined due to Harish-Chandra's result on the differentiability of $\Pi$ on $\mathfrak{X}_{K},([\mathrm{H}-\mathrm{Ch} 1])$. Then $\left(\dot{\pi}, \mathfrak{X}_{K}\right)$ is called the Harish-Chandra module associated to $(\Pi, \mathfrak{X})$.

Two admissible representations of $G$ are called in finitesimally equivalent if their corresponding Harish-Chandra modules are equivalent $(\mathfrak{g}, K)$-modules.

(4.1.6) We adopt the common definition of submodule for the category $\mathfrak{c}(\mathfrak{g}, K)$. However, we say $(\Pi, \mathfrak{W})$ is a $G$-subrepresentation of the representation $(\Pi, \mathfrak{X})$ if $\mathfrak{W}$ is a $G$-invariant closed subspace of $\mathfrak{X}$. Based on this, we define irreducible, reducible, and indecomposable $(\mathfrak{g}, K)$-module (resp. $G$-representation) in the usual way.

The following properties of the Harish-Chandra correspondence justify the attention paid to the category $\mathfrak{c}(\mathfrak{g}, K)$, and the identification of a $G$-representation with its Harish-Chandra module in some occasions.

Theorem 4.1.7 ([W-1], [W-2]). Let $(\Pi, \mathfrak{X})$ be a representation of $G$.

(a) If $(\Pi, \mathfrak{X})$ is admissible, the Harish-Chandra correspondence (4.1.5) establishes a bijection between $G$-subrepresentations of $(\Pi, \mathfrak{X})$ and the $(\mathfrak{g}, K)$ submodules of $\left(\dot{\pi}, \mathfrak{X}_{K}\right)$. In particular, $(\Pi, \mathfrak{X})$ is irreducible if and only if $\left(\dot{\pi}, \mathfrak{X}_{K}\right)$ is irreducible. 
(b) If $(\Pi, \mathfrak{X})$ is irreducible unitary then it is admissible.

(c) Any irreducible object in $\mathfrak{c}(\mathfrak{g}, K)$ is in $\mathfrak{a}(\mathfrak{g}, K)$, and it is the HarishChandra module of some admissible representation of $G$ (necessarily irreducible by (a)).

(d) Two irreducible unitary representations of $G$ are unitarily equivalent if and only if they are infinitesimally equivalent.

Definition 4.1.8 (Notation (4.1.5)). Let $(\Pi, \mathfrak{X})$ be an admissible $G$-representation.

(a) $(\Pi, \mathfrak{X})$ is said to be of finite length if $\left(\dot{\pi}, \mathfrak{X}_{K}\right)$ is in $\mathfrak{F}(\mathfrak{g}, K)$.

(b) $(\Pi, \mathfrak{X})$ is completely reducible if $\left(\dot{\pi}, \mathfrak{X}_{K}\right)$ is a direct sum of $(\mathfrak{g}, K)$ submodules that are irreducible.

(4.1.9) Now, fix $K$ as above, let $G=K A N$ be an Iwasawa decomposition, and let $P=M A N$ be the corresponding minimal parabolic subgroup. From definition (2.3), $P$ determines a positive root system $\bar{\Delta}^{+}=\bar{\Delta}(P, A)$ for the reduced root system $\bar{\Delta}$. The collection of simple roots of $\bar{\Delta}^{+}$will be denoted by $\bar{\Delta}_{o}$. For any subset $F \subseteq \bar{\Delta}_{o}$, we build up a parabolic subgroup $P_{F}$ of $G$ containing $P$.

First, we set

$$
\begin{aligned}
A_{F} & =\{a \in A \mid \alpha(a)=1 \text { for all } \alpha \in F\} \\
\bar{M}_{F} & =\text { centralizer of } A_{F} \text { in } G .
\end{aligned}
$$

Next, define $\Delta_{F}=\left\{\alpha \in \bar{\Delta}^{+} \mid \alpha(a) \neq 1\right.$ for some $\left.a \in A_{F}\right\}$, and set

$$
N_{F}=\exp \left(\sum_{\alpha \in \Delta_{F}}\left(\mathfrak{g}_{o}\right)_{\alpha}\right) \subseteq N
$$

where $\sum_{\alpha \in \Delta}\left(\mathfrak{g}_{o}\right)_{\alpha}$ is the root space decomposition of $\operatorname{Lie}(N)$ with respect to $\bar{\Delta}_{o}$.

We get $P_{F}=\bar{M}_{F} N_{F}$ is a parabolic subgroup of $G$ with Levi factor $\bar{M}_{F}$. On the other hand, $\bar{M}_{F}$ can be decomposed as $M_{F} A_{F}$ for some group $M_{F}$ in the Harish-Chandra class of compact center ([W-2]). Thus, we obtain the Langlands decomposition of $P_{F}, P_{F}=M_{F} A_{F} N_{F}$.

It is known that in any conjugate class of parabolic subgroups of $G$ there is a unique representative of the form $P_{F}$ for some $F$.

Definition 4.1.10. A pair $\left(P_{F}, A_{F}\right)$ constructed above for some $F \subseteq \bar{\Delta}_{o}$ is called a P-pair.

(The $P$-pair for $F=\phi$ is $(P, A)$ and in this case $\bar{M}_{F}=M A$.) We denote by $\Delta\left(P_{F}, A_{F}\right)$ the set of roots of $\mathfrak{a}_{F}=\operatorname{Lie}\left(A_{F}\right) \otimes \mathbb{C}$ in $\mathfrak{n}_{F}=\operatorname{Lie}\left(N_{F}\right) \otimes \mathbb{C}$.

Let $\left(P_{F}, A_{F}\right)$ be a $P$-pair with Langlands decomposition $P_{F}=M_{F} A_{F} N_{F}$. We obtain representations of $G$ from representations of $P_{F}$ through the real parabolic induction construction $\operatorname{Ind}_{P_{F}}^{G}()$. The case we are mainly interested in working on is $\operatorname{Ind}_{P_{F}}^{G}(\gamma \otimes \omega \otimes 1)$, where $\gamma$ is an admissible representation of $M_{F}$ of finite length, $\omega$ is in $\widehat{A_{F}}$, and $N_{F}$ is acting trivially on $\gamma \otimes \omega \otimes 1$.

Notation 4.1.11. We write $\operatorname{Ind}_{P_{F}}^{G}(\gamma \otimes \omega)$ instead of $\operatorname{Ind}_{P_{F}}^{G}(\gamma \otimes \omega \otimes 1)$, and when $\omega=1$, simply as $\operatorname{Ind}_{P_{F}}^{G}(\gamma \otimes 1)$.

For $\operatorname{Ind}_{P_{F}}^{G}(\gamma \otimes \omega)$ in the previous situation we have the following properties. 
Proposition 4.1.12 ([Green], Chapter 4). We have the following properties for real parabolic induction:

(a) $\operatorname{Ind}_{P_{F}}^{G}(\gamma \otimes \omega)$ is an admissible representation of $G$.

(b) If $\gamma \otimes \omega$ is a unitary representation of $M_{F} A_{F}$ then $\operatorname{Ind}_{P_{F}}^{G}(\gamma \otimes \omega)$ is unitary and completely reducible.

(c) If $\gamma$ is a discrete series or limit of discrete series representation of $M_{F}$ then $\operatorname{Ind}_{P_{F}}^{G}(\gamma \otimes \omega)$ has finite length.

(d) If $P_{F_{1}}$ and $P_{F_{2}}$ are two parabolic subgroups having the same Levi part $M_{F} A_{F}$ then the induced representations $\operatorname{Ind}_{P_{F_{1}}}^{G}(\gamma \otimes \omega)$ and $\operatorname{Ind}_{P_{F_{2}}}^{G}(\gamma \otimes \omega)$ have the same composition factors.

Notation 4.1.13. We will write either

$$
X_{G}(\gamma \otimes \omega) \text { or } X_{G}\left(P_{F}, \gamma \otimes \omega\right)
$$

for the Harish-Chandra module associated to $\operatorname{Ind}_{P_{F}}^{G}(\gamma \otimes \omega)$.

We will need the following property of parabolic induction whose proof can be found in [Green], Lemma (4.1.17).

Proposition 4.1.14 (Induction by stages). Let $P$ be a minimal parabolic subgroup of $G$, and let $\bar{\Delta}_{o}$ be as in Definition (4.1.9). Consider $F_{1} \subseteq F_{2} \subseteq \bar{\Delta}_{o}$. Let $\left(P_{F_{1}}, A_{F_{1}}\right)$ and $\left(P_{F_{2}}, A_{F_{2}}\right)$ be the two corresponding P-pairs (Definition (4.1.10)), with Langlands decompositions $P_{F_{1}}=M_{F_{1}} A_{F_{1}} N_{F_{1}}$ and $P_{F_{2}}=M_{F_{2}} A_{F_{2}} N_{F_{2}}$. Thus, we have $P \subseteq P_{F_{1}} \subseteq P_{F_{2}}, P_{F_{1}} \cap M_{F_{2}}$ is a parabolic subgroup of $M_{F_{2}}$, and $A_{F_{1}}=A_{F_{2}}\left(A_{F_{1}} \cap M_{F_{2}}\right)$. Suppose $\gamma$ is an admissible representation of $M_{F_{1}}$, and $\nu$ is a representation of $A_{F_{1}}$. Write $\nu_{1}=\left.\nu\right|_{A_{F_{1}} \cap M_{F_{2}}}$ and $\nu_{2}=\left.\nu\right|_{A_{F_{2}}}$. Then we obtain

$$
\operatorname{Ind}_{P_{1}}^{G}(\gamma \otimes \nu)=\operatorname{Ind}_{P_{F_{2}}}^{G}\left(\operatorname{Ind}_{P_{F_{1}} \cap M_{F_{2}}}^{M_{F_{2}}}\left(\gamma \otimes \nu_{1}\right) \otimes \nu_{2}\right) .
$$

To formulate the Langlands classification as in the introduction, we need the parabolic subgroups to be in certain position with respect to the representations we are inducing on. We identify in the natural way $\widehat{A}$ with $\left(\mathfrak{a}_{o}\right)^{*}$ for $\operatorname{Lie}(A)=$ $\mathfrak{a}_{o}$, in the following definition ([Green]).

Definition 4.1.15. Let $P=M A N$ be a minimal parabolic subgroup, $\bar{\Delta}^{+}$and $\bar{\Delta}_{o}$ corresponding to $P$, as in Definition (4.1.9). Given $F \subseteq \bar{\Delta}_{o}, \nu \in \widehat{A_{F}}$ is called negative for $P_{F}$ (or $P_{F}$ negative for $\nu$ ) if for all $\alpha \in \bar{\Delta}^{+}, \operatorname{Re}(\langle\alpha, \nu\rangle)$ $\leq 0$. Put $F_{1}=\left\{\alpha \in \bar{\Delta}_{o} \mid \operatorname{Re}(\langle\alpha, \nu\rangle)=0\right\}$. If $\nu$ is negative for $P_{F_{1}}$, the $P$-pair $\left(P_{F_{1}}, A_{F_{1}}\right)$ is said to be defined by $(-\operatorname{Re} \nu)$.

(4.1.16) Let $\left(P_{F}, A_{F}\right)$ be a $P$-pair with Langlands decomposition $P_{F}=$ $M_{F} A_{F} N_{F}$. When $\omega$ is a unitary character of $A_{F}$ and $\gamma$ is a discrete series representation of $M_{F}$, we have from (4.1.12) (d) that $\operatorname{Ind}_{P_{F}}^{G}(\gamma \otimes \omega)=$ $\operatorname{Ind}_{P_{F_{1}}}^{G}(\gamma \otimes \omega)$ for any parabolic subgroup $P_{F_{1}}$ containing $M_{F} A_{F}$. Such a representation $\operatorname{Ind}_{P_{F}}^{G}(\gamma \otimes \omega)$ will be called a standard tempered representation.

Definition 4.1.17. An irreducible admissible representation of $G$ is called tempered if it is a direct summand of some standard tempered representation (4.1.16). 
A reason for giving Definition (4.1.15) is that $\operatorname{Ind}_{P_{F}}^{G}(\gamma \otimes \nu)$ has a unique irreducible submodule when the $P$-pair $\left(P_{F}, A_{F}\right)$ is defined by $(-\operatorname{Re} \nu)$ and $\gamma$ is any irreducible tempered representation of $M_{F}$. The statement in the introduction on the Langlands classification makes use of this fact ([W-2], (5.4.1)).

In case $G$ has compact center, Proposition 3.7 given in [B-W], Chapter IV asserts that an irreducible representation $(\Pi, \mathfrak{X})$ of $G$ is tempered if and only if for any pair of $K$-finite vectors $x$ and $y$, the matrix coefficient $\langle\Pi(g) x, y\rangle$ : $G \rightarrow \mathbb{C}$ is in $L^{2+\epsilon}(G) \forall \epsilon>0$.

Definition 4.1.18. For a discrete series representation $\delta$, and $\nu \in \widehat{A_{F}}$ is negative for $P_{F}$, the induced representation $\operatorname{Ind}_{P_{F}}^{G}(\delta \otimes \nu)$ is called a standard representation.

Definition 4.1.19. For an induced representation $\operatorname{Ind}_{P_{F}}^{G}(\gamma \otimes \omega)$ where $\gamma$ is an irreducible tempered representation and $P_{F}$ is negative for $\omega$, we define its Langlands subrepresentation to be the subrepresentation of $G$ associated to the $(\mathfrak{g}, K)$-submodule of $X_{G}(\gamma \otimes \omega)$ which is maximal completely reducible, denoted by $\bar{X}_{G}(\gamma \otimes \omega)$, under the Harish-Chandra correspondence. According to (4.1.7) (a), we identify the Langlands subrepresentation with $\bar{X}_{G}(\gamma \otimes \omega)$. Note that the Harish chandra module of a standard tempered representation satisfies $X_{G}(\gamma \otimes \omega)=\bar{X}_{G}(\gamma \otimes \omega)$.

(4.1.20) To classify tempered representations of $G$, we will give an explicit decomposition into irreducible representations for any standard tempered representation.

(4.1.21) It is our main purpose to find an explicit decomposition of any Langlands subrepresentation. This seems more general than classifying tempered representations, but actually both tasks are equivalent: Let $\operatorname{Ind}_{P_{F}}^{G}(\delta \otimes \nu)$ be a standard representation. Take $F_{1} \subseteq \bar{\Delta}_{o}$ such that the $P$-pair $\left(P_{F_{1}}, A_{F_{1}}\right)$ is defined by $-\operatorname{Re} \nu$. We apply induction by stages to $\operatorname{Ind}_{P_{F}}^{G}(\delta \otimes \nu)$,

$$
\operatorname{Ind}_{P_{F}}^{G}(\delta \otimes \nu)=\operatorname{Ind}_{P_{F_{1}}}^{G}\left(\operatorname{Ind}_{P_{F} \cap M_{F_{1}}}^{M_{F_{1}}}\left(\gamma \otimes \nu_{1}\right) \otimes \nu_{2}\right)
$$

where $\nu_{1}=\left.\nu\right|_{A_{F} \cap M_{F_{1}}}$ and $\nu_{2}=\left.\nu\right|_{A_{F_{1}}} . \quad \operatorname{Ind}_{P_{F} \cap M_{F_{1}}}^{M_{F_{1}}}\left(\gamma \otimes \nu_{1}\right)$ is a standard tempered representation. Thus, if we know the decomposition for this representation then we know the decomposition for the Langlands subrepresentation of $\operatorname{Ind}_{P_{F}}^{G}(\delta \otimes \nu)$. This is so, since for any irreducible tempered representation $\sigma$ the Langlands subrepresentation of $\operatorname{Ind}_{P_{F_{1}}}^{G}\left(\sigma \otimes \nu_{2}\right)$ is irreducible.

4.2 Minimal $K$-types and $\lambda$-minimal $K$-types. Suppose $G$ is the HarishChandra class with maximal compact subgroup $K$. Take $T^{c}$ a maximal torus of $K$. Put $\mathfrak{k}_{o}=\operatorname{Lie}(K)$ and $\mathfrak{t}_{o}^{c}=\operatorname{Lie}\left(T^{c}\right)$. Fix a positive root system $\Delta_{c}^{+}$for $\Delta\left(\mathfrak{k}, \mathfrak{t}^{c}\right)$ for this subsection, and define $\rho_{c}=\rho\left(\Delta_{c}^{+}\right)$.

By the classification of the representations of $K_{o}$, we write any element of $\widehat{K_{o}}$ as $\pi_{\mu}$ where $\mu \in \widehat{T_{o}^{c}}$ is its highest weight (w.r.t. $\Delta_{c}^{+}$). To determine $\widehat{K}$ (for $K$ disconnected), we need the normalizer of $T^{c}$ in $K$, denoted by $N_{K}\left(T^{c}\right)$. We note the property $K=N_{K}\left(T^{c}\right) K_{o}$. Set

$$
\mathfrak{N}_{K}=\left\{n \in N_{K}\left(T^{c}\right) \mid n \cdot \rho_{c}=\rho_{c}\right\} .
$$


Consider $\mu \in \widehat{T_{o}^{c}}$. Since $T_{o}^{c}$ is a normal subgroup of $\mathfrak{N}_{K}$, we are in situation (2.15) with the triple $\left(\mathfrak{N}_{K}, T_{o}^{c}, \mu\right)$. The stabilizer of $\mu$ in $\mathfrak{N}_{K}$ is denoted by $\left(\mathfrak{N}_{K}\right)_{\mu}$. With respect to $\Delta_{c}^{+}$we assume $\mu \in \widehat{T_{o}^{c}}$ is a dominant weight. Then we write

$$
\widehat{K}(\mu)=\left\{\pi \in \widehat{K} \mid \pi_{\mu} \in \widehat{K_{o}} \text { occurs }\left.\in \pi\right|_{K_{o}}\right\}
$$

We have certain generalization of the highest weight representation.

Proposition 4.2.1. With respect to $\Delta_{c}^{+}$, suppose $\mu \in \widehat{T}_{o}^{c}$ is a dominant weight.

(a) For $\pi \in \widehat{K}(\mu)$, the highest weights of the elements of $\widehat{K_{o}}$ occurring in $\left.\pi\right|_{K_{o}}$ are of the form $x \cdot \mu$ for some $x \in \mathfrak{N}_{K}$.

(b) There is a one-to-one correspondence between $\widehat{K}(\mu)$ and the set

$\mathfrak{J}:=\left\{\gamma\right.$ is an irreducible representation of $\left(\mathfrak{N}_{K}\right)_{\mu} \mid \mu$ occurs in $\left.\left.\gamma\right|_{T_{o}^{c}}\right\}$.

Proof. By the Frobenius reciprocity theorem, $\widehat{K}(\mu)=\{\pi \in \widehat{K} \mid \pi$ occurs in $\left.\operatorname{Ind}_{K_{o}}^{K}\left(\pi_{\mu}\right)\right\}$. On one hand, $\left.\operatorname{Ind}_{T_{o}^{c}}^{\mathfrak{N}_{K}}(\mu)\right|_{T_{o}^{c}}$ is the direct sum of the highest weights of the constituents of $\left.\operatorname{Ind}_{K_{o}}^{K}\left(\pi_{\mu}\right)\right|_{K_{o}}$. Thus, (a) follows from (2.17) (b), and $\widehat{K}(\mu)$ is in bijection with the set $\left\{\gamma \in \widehat{\mathfrak{N}_{K}} \mid \gamma\right.$ occurs in $\left.\operatorname{Ind}_{T_{o}^{c}}^{\mathfrak{N}_{K}}(\mu)\right\}$. The functor $\operatorname{Ind}_{\left(\mathfrak{N}_{K}\right)_{\mu}}^{\mathfrak{N}_{K}}()$, on the other hand, gives a bijection between $\mathfrak{J}$ and the latter set. Therefore, (b) holds.

Now, denote $\bar{\mu}$ for the differential $d \mu$. Write $\|\gamma\|=\left\langle\gamma+2 \rho_{c}, \gamma+2 \rho_{c}\right\rangle$ for any $\gamma \in\left(i t_{o}^{c}\right)^{*}$ (which is not a negative real number).

Consider $\pi \in \widehat{K}(\mu)$. By Proposition (4.2.1), all its highest weights are of the form $x \cdot \mu$ for some $x \in \mathfrak{N}_{K}$. Since $x \cdot \rho_{c}=\rho_{c}$, we can use any of these to define $\|\pi\|:=\|\bar{\mu}\|$.

Definition 4.2.2. Let $X$ be a non-zero Harish-Chandra module for $G$. The set of minimal $K$-types of $X$ is defined by

$$
\begin{aligned}
\left\{(\pi, V) \in \widehat{K} \mid \operatorname{dim}\left(\operatorname{Hom}_{K}(V, X)\right) \neq 0,\right. & \text { and }\|\pi\| \text { is minimal with this property }\}
\end{aligned}
$$

Since $\langle$,$\rangle is positive definite on the weight lattice in \left(i t_{0}^{c}\right)^{*}$ and $X$ is admissible, the set of minimal $K$-type of $X$ is non-empty and finite.

Proposition 4.2.3. Suppose $G$ is quasisplit (Definition (2.8)), $\delta$ is a fine representation of $\widehat{M}$ and $\nu \in \widehat{A}$ is unitary. Let $X$ be the Harish-Chandra module of any irreducible tempered representation appearing as direct summand in the decomposition of $X_{G}(\delta \otimes \nu),(4.1 .17)$. Then every minimal $K$-types of $X$ is a $K$-fine representation (2.13).

Proof. In [V-I], the result is proved when $G$ is connected. Remark (2.14) reduces the general case to this situation.

Proposition (4.2.3) implies that $A(\delta)$ (Definition (2.24)) is non-empty.

(4.2.4) Another way to order the $K$-types of Harish-Chandra modules is based in the Vogan calculus. For any $\mu \in \widehat{T_{o}^{c}}$ dominant, Vogan associates to it a unique element $\lambda(\mu) \in\left(i \mathfrak{t}_{o}^{\mathfrak{c}}\right)^{*}$ which is dominant with respect to $\Delta_{c}^{+}$([Green], Chapter 5). Hence, for any $\mu \in \widehat{T_{o}^{c}}$ define $\|\mu\|_{\lambda}=\langle\lambda(\mu), \lambda(\mu)\rangle$. 
It is possible to prove the property $\lambda(x \mu)=x \lambda(\mu)$ for each $x \in \mathfrak{N}_{K}$. Therefore, in view of Proposition (4.2.1) (a), there is no ambiguity in defining for any $\pi \in \widehat{K}\left(\mu^{\prime}\right)$

$$
\|\pi\|_{\lambda}=\left\langle\lambda\left(\mu^{\prime}\right), \lambda\left(\mu^{\prime}\right)\right\rangle .
$$

Definition 4.2.5. Let $X$ be a Harish-Chandra module for $G$. The set of $\lambda$ minimal $K$-types of $X$ is defined by

$$
\begin{aligned}
\left\{(\pi, V) \in \widehat{K} \mid \operatorname{dim}\left(\operatorname{Hom}_{K}(V, X)\right) \neq 0,\right. \\
\text { and } \left.\|\pi\|_{\lambda} \text { is minimal with this property }\right\} .
\end{aligned}
$$

(Definitions (4.2.2) and (4.2.5) do not coincide for arbitrary Harish-Chandra modules; however, they are equivalent for irreducible representations ([V-I], Lemma 8.8).) Now, consider the root space decomposition for $\mathfrak{g}$ with respect to $\mathfrak{h}^{c}$, a $\theta$-stable Cartan subalgebra containing $\mathfrak{t}^{c}$.

$$
\mathfrak{g}=\mathfrak{h}^{c}+\sum_{\alpha \in \Delta\left(\mathfrak{g}, \mathfrak{h}^{c}\right)} \mathfrak{g}_{\alpha} .
$$

While for real parabolic induction we use $(-\theta)$-stable parabolic subalgebras of $\mathfrak{g}$ (namely, the Lie algebras of parabolic subgroups of $G$ ), for the cohomological induction construction we need to consider $\theta$-stable parabolic subalgebras.

Definition 4.2.6 ([Green], Chapter 5). For any $\lambda \in\left(i t_{o}^{c}\right)^{*}$ dominant with respect to $\Delta_{c}^{+}$, we construct the subalgebras $\mathfrak{l}$ and $\mathfrak{u}$ :

$$
\mathfrak{l}=\mathfrak{h}^{c}+\sum_{\alpha \in \Delta\left(\mathfrak{g}, \mathfrak{h}^{c}\right),\langle\alpha, \lambda\rangle=0} \mathfrak{g}_{\alpha} \quad \text { and } \quad \mathfrak{u}=\sum_{\alpha \in \Delta\left(\mathfrak{g}, \mathfrak{h}^{c}\right),\langle\alpha, \lambda\rangle>0} \mathfrak{g}_{\alpha} .
$$

The $\theta$-stable parabolic subalgebra of $\mathfrak{g}$ given by $\mathfrak{q}(\lambda)=\mathfrak{q}=\mathfrak{l}+\mathfrak{u}$ is called the $\theta$-stable parabolic subalgebra of $\mathfrak{g}$ associated to $\lambda$.

(4.2.7) Let $\mathfrak{q}=\mathfrak{l}+\mathfrak{u}$ be the $\theta$-stable parabolic subalgebra associated to $\lambda(\mu)$ with $\mu \in \widehat{T}_{o}^{c}$ dominant, and let $L$ be the normalizer of $q$ in $G$. It turns out that $L \cap K$ is a maximal compact subgroup of $L$ containing $T^{c}$ as maximal torus. Write $\Delta_{c}^{+}(\mathfrak{l})=\Delta\left(\mathfrak{l} \cap \mathfrak{k}, \mathfrak{t}^{c}\right) \cap \Delta_{c}^{+}$. As in (4.2.4), the sets $(\widehat{L \cap K})(\mu)$ and $\mathfrak{N}_{L \cap K}$ are defined for $L$ instead of $G$. If $x \in N_{K \cap L}\left(T^{c}\right)=\left(N_{K}\left(T^{c}\right) \cap L\right)$ then $x \cdot \rho(\mathfrak{u} \cap \mathfrak{k})=\rho(\mathfrak{u} \cap \mathfrak{k})$. Thus, if we multiply both sides of $\rho_{c}=\rho\left(\Delta_{c}^{+}(\mathfrak{l})\right)+\rho(\mathfrak{u} \cap \mathfrak{k})$ by this $x$, we have

$$
x \cdot \rho_{c}=\rho_{c} \quad \text { if and only if } x \cdot \rho\left(\Delta_{c}^{+}(\mathfrak{l})\right)=\rho\left(\Delta_{c}^{+}(\mathfrak{l})\right)
$$

We conclude that $\mathfrak{N}_{K} \cap L=\mathfrak{N}_{L \cap K}$. In particular, $\left(\mathfrak{N}_{L \cap K}\right)_{\mu}=\left(\mathfrak{N}_{K} \cap L\right)_{\mu}$. In fact, more is true:

Claim. $\left(\mathfrak{N}_{K}\right)_{\mu}=\left(\mathfrak{N}_{L \cap K}\right)_{\mu}$.

To prove this statement is enought to prove $\left(\mathfrak{N}_{K}\right)_{\mu} \subseteq L$, for this implies that $\left(\mathfrak{N}_{K}\right)_{\mu}$ is contained in $\left(\mathfrak{N}_{K} \cap L\right)_{\mu}$. But any $x \in\left(\mathfrak{N}_{K}\right)_{\mu}$ satisfies $x \lambda(\mu)=$ $\lambda(x \mu)=\lambda(\mu)$, in view of the property of $\lambda(\mu)$ in (4.2.4). This implies that $x \in L$. 
Corollary 4.2.8. For any $\mu \in \widehat{T_{o}^{c}}$ dominant, let $\mathfrak{q}$ be $\theta$-stable parabolic subalgebra of $\mathfrak{g}$ associated to $\lambda(\mu)$ (4.2.4). Then there is a bijection between the sets $\widehat{K}(\mu)$ and $\widehat{L \cap K}(\mu)$.

Proof. This result is consequence of (4.2.4) and that $\left(\mathfrak{N}_{K}\right)_{\mu}=\left(\mathfrak{N}_{L \cap K}\right)_{\mu}$ holds.

\subsection{Proof of Proposition (3.5).}

Example 4.3.1. Let $G$ be a group in the Harish-Chandra class satisfying the following conditions:

(a) The center of $G$ is compact,

(b) $\operatorname{Lie}(G)=\mathfrak{g}_{o}=\mathfrak{g}_{o}^{1}+\mathfrak{g}_{o}^{2}+\cdots+\mathfrak{g}_{o}^{n}+\mathfrak{c}_{o}$

where $\mathfrak{c}_{o}$ is the center of $\mathfrak{g}_{o}$ and $\mathfrak{g}_{o}^{i} \cong \operatorname{sl}(2, \mathbb{R}) ; i=1,2, \cdots, n$. Therefore, $\mathfrak{h}^{s}=\mathfrak{a}^{s}+\mathfrak{c}$ is a split Cartan subalgebra of $\mathfrak{g}$ (Definition (2.3)), such that $\mathfrak{a}_{o}^{s} \cap \mathfrak{g}_{o}^{i}$ is a split Cartan subalgebra of $\mathfrak{g}_{o}^{i}$. We choose a set of positive roots $\Delta^{+}(\mathfrak{g}, \mathfrak{a})=$ $\left\{\alpha_{1}, \cdots, \alpha_{n}\right\}$ which is a strongly orthogonal set of real roots. $W=M^{\prime} / M=$ $(\mathbb{Z} / 2 \mathbb{Z})^{n}$ is the corresponding Weyl group. Recall the definition of $\sigma_{\alpha_{i}}$ and $m_{\alpha_{i}}$ (Definition (2.7)); $i=1,2, \cdots, n$. Assume $\delta \in \widehat{M}$ is a fine representation such that $\delta\left(m_{\alpha_{i}}\right)=-I$ for all $i=1, \cdots, n$. In the notation given in Definition (2.28), the assumptions above imply that the set of good roots $\bar{\Delta}_{\delta}$ for $\delta$ is empty, and $\left(M_{\delta}^{\prime}\right)^{o}=M$. Thus, we have $R_{\delta}=M_{\delta}^{\prime} / M=W_{\delta} \subseteq W$.

Lemma 4.3.2. Under the conditions stated in Example (4.3.1) (and notation in Propositions (3.2) and (3.5)), the following holds:

(a) The $R$-group $\widehat{R_{\delta}}$ acts on $A_{d}(\delta)$ transitively.

(b) $X_{G}(\delta \otimes 1)=m(\delta, \mu) \sum_{\mu \in A(\delta)} I_{\mu}$, where $I_{\mu}$ is an irreducible admissible (g,K)-module with unique minimal $K$-type $\mu$. Moreover, $\mu \cong \mu^{\prime}$ in $\widehat{K}$ if and only if $I_{\mu}$ and $I_{\mu^{\prime}}$ are equivalent $(\mathfrak{g}, K)$-modules. $I_{\mu}$ appears in the sum $m(\delta, \mu)$-times.

Proof. To prove (a), it suffices to show that $\left|\widehat{R_{\delta}^{1}}\right|=\left|A_{d}(\delta)\right|$ by proposition (3.2).

Case 1. Let us prove Lemma (4.3.2) (a) for $G^{\#}$ (Notation (1.2)).

In this case, $K^{\#}=T^{c}$. By Proposition (4.2.3), let $\mu$ be a non-zero element in $A(\delta)$. We fix a system of positive roots $\Delta^{+}\left(\mathfrak{g}, \mathfrak{t}^{c}\right)=\left\{\beta_{1}, \cdots, \beta_{n}\right\}$ making $(-\mu)$ dominant. Identify $\left\{\beta_{1}, \cdots, \beta_{n}\right\}$ with their corresponding analytic elements in $\widehat{T^{c}}$. These elements are extensions of the characters in $\widehat{W}$. Now define

$$
\mathbb{A}_{\mu}:=\left\{\beta_{i_{1}} \otimes \cdots \otimes \beta_{i_{t}} \otimes \mu \mid i_{1}<i_{2}<\cdots<i_{t} ; \text { where } i_{j} \in\{1,2, \cdots, n\}\right\} .
$$

$\mathbb{A}_{\mu}$ contains $2^{n} T^{c}$-fine representations. Proposition (3.2) implies that $A(\delta) \subseteq$ $\mathbb{A}_{\mu}$. But $\left.\beta_{i_{1}} \otimes \cdots \otimes \beta_{i_{t}} \otimes \mu\right|_{M}=\left.\mu\right|_{M}$ implies that any element of $\mathbb{A}_{\mu}$ restricted to $M$ contains $\delta$ as submodule. Therefore, $A(\delta)=\mathbb{A}_{\mu}$ and $|A(\delta)|=2^{n}$. This implies $2^{n}=\left|A_{d}(\delta)\right| \leq\left|\widehat{R_{\delta}^{1}}\right| \leq 2^{n}$. Then (a) holds in this case. Consequently, we obtain that $\widehat{R_{\delta}^{1}}=\widehat{R_{\delta}}=\widehat{W}, M_{\delta}^{\prime}=M^{\prime},\left|\widehat{R_{\delta}^{o}}\right|=1$ and $\widehat{W}_{\mu}=1$.

Case 2. We reduce the general case to the Case 1.

Denote, $M_{T}=M \cap T^{c}$ and $M_{T}^{\prime}=M^{\prime} \cap T^{c}$. Again we make use of Proposition (4.2.3) to fix an element $\mu$ in $A(\delta)$. Let $\delta_{o}$ be in $\widehat{M_{T}}$ occurring in $\left.\delta\right|_{M_{T}}$. By Remark (2.14), $\delta_{o}$ is fine. Hence, Case 1 implies that $A\left(\delta_{o}\right)=\mathbb{A}_{\mu_{o}}$ for some $\mu_{o} \in A\left(\delta_{o}\right)$. Choose $\mu_{o}$ such that it is a direct summand of $\left.\mu\right|_{T^{c}}$. We write 
$W_{c}$ for the analytic Weyl group $K / T^{c}$. This group is isomorphic to $(\mathbb{Z} / 2 \mathbb{Z})^{m}$ for some $m$. $\widehat{W}_{c}$ acts on $\widehat{K}$, and $W_{c}$ acts on $\mathbb{A}_{\mu_{o}}$. Clearly, the stabilizer of $\mu_{o}$ in $K$ is exactly $T^{c}$ (i.e., the stabilizer of $\mu_{o}$ in $W_{c}$ is exactly the trivial group); equivalently $\left(\widehat{W}_{c}\right)_{\mu}=\widehat{W}_{c}$ and $m\left(\mu_{o}, \mu\right)=1$ (Lemma (3.8) (c)).

Define $R=M^{\prime} / M, V=M_{T}^{\prime} / M_{T}, S=M^{\prime} / M_{T}^{\prime}$ and $U=M / M_{T}$; thus, $W=R \cong V$ and $W_{c} \cong S \cong U$.

Without writing restriction, we regard $\mu$ as an element of $\widehat{M}^{\prime}$, and $\mu_{o}$ as an element of $\widehat{M_{T}^{\prime}}$ based on Corollary (2.22). Thus, we get $\widehat{S}_{\mu}=\widehat{S}$ and $m\left(\mu_{o}, \mu\right)=1$. Moreover, from Case $1\left(\right.$ and $\left.\left|\mathbb{A}_{\mu_{o}}\right|=2^{n}\right), \widehat{V}_{\mu_{o}}=1$ and $\left(M_{T}^{\prime}\right)_{\delta_{o}}=$ $M_{T}^{\prime}$.

Claim. $m\left(\delta_{o}, \delta\right)=m(\delta, \mu)$.

Sublemma. The following two sequences

$$
\begin{gathered}
1 \rightarrow \widehat{R}_{\mu} \rightarrow M_{\delta_{o}}^{\prime} / M_{T}^{\prime} \rightarrow 1, \\
1 \rightarrow \widehat{U}_{\delta} \rightarrow \widehat{U} \rightarrow M^{\prime} / M_{\delta}^{\prime} \rightarrow 1
\end{gathered}
$$

are exact.

Assume for a moment this sublemma is true. In view of $\left(M_{T}^{\prime}\right)_{\delta_{o}}=M_{T}^{\prime}$, this implies $M_{\delta_{o}}^{\prime} / M_{T}^{\prime} \cong M_{\delta_{o}} / M_{T}$. Thus, as a consequence of sublemma (ii) we have

$$
\left|M^{\prime} / M_{\delta}^{\prime}\right|\left|\widehat{U}_{\delta}\right|=|\widehat{U}|=\left|M / M_{\delta_{o}}\right|\left|M_{\delta_{o}} / M_{T}\right|=\left|M / M_{\delta_{o}}\right|\left|\widehat{R}_{\mu}\right|
$$

The last equality is an application of sublemma (i). So, $\left|M / M_{\delta_{o}}\right|^{-1}\left|\widehat{U}_{\delta}\right|=$ $\left|M^{\prime} / M_{\delta}^{\prime}\right|^{-1}\left|\widehat{R}_{\mu}\right|$. By Corollary (3.9) (b), the claim $m\left(\delta_{o}, \delta\right)=m(\delta, \mu)$ follows.

On the other hand, since $G / P=G^{\#} / P^{\#}$, we can write

$$
\left.\operatorname{Ind}_{P}^{G}(\delta \otimes 1)\right|_{G^{*}}=m\left(\delta_{o}, \delta\right) \sum_{x \in M / M_{\delta_{o}}} \operatorname{Ind}_{P^{*}}^{G^{*}}\left(x \cdot \delta_{o} \otimes 1\right) .
$$

The number of $T^{c}$-fine representations on the left is equal to $m(\delta, \mu) \cdot|A(\delta)| \cdot|S|$ $\left(|S|=\left|W_{c}\right|\right.$ is the number of $T^{c}$-fine representations appearing in $\left.\mu\right|_{T^{c}}$ for any $\mu \in A(\delta)$, since $\left.\left(W_{c}\right)_{\mu_{o}}=1\right)$. Meanwhile, by Case 1 there are $m\left(\delta_{o}, \delta\right) \mid M /$ $M_{\delta_{o}}|| R \mid T^{c}$-fine representations on the right. This and the claim above imply

$$
|R|\left|M / M_{\delta_{o}}\right|=|A(\delta)||S|=|A(\delta)|\left|M / M_{\delta_{o}}\right|\left|M_{\delta_{o}} / M_{T}\right|=|A(\delta)|\left|\widehat{R}_{\mu}\right|\left|M / M_{\delta_{o}}\right|
$$

where the last part is an application of sublemma (i). Conclude that $\left|\widehat{R_{\delta}^{1}}\right|=$ $\left|A_{d}(\delta)\right|$ by Corollary (3.9) (a) and Remark (2.24).

To finish the proof of (a) of the lemma, we prove the sublemma:

We have $\widehat{V}$ acts on $\widehat{M_{T}^{\prime}}$ and $\widehat{R}$ acts on $\widehat{M}^{\prime}$. We also have $\widehat{S}$ acts on $\widehat{M}^{\prime}$ and $\widehat{U}$ acts on $\widehat{M}$. Regard again $\mu$ as an element of $\widehat{M}^{\prime}$, and $\mu_{o}$ as an element of $\widehat{M_{T}^{\prime}}$.

(i) First we observe the isomorphism $\widehat{R} \cong \widehat{V}$ is given by restricting characters. In this statement, for any $\chi \in \widehat{R}$ we write $\chi \mid$ for the corresponding character in $\widehat{V}$. First, note that if we take $\chi \in \widehat{R}$ then $\chi \mid \otimes \mu_{o}$ occurs in the restriction of $\mu$ to $M_{T}^{\prime}$ if and only if there is an $s \in M^{\prime}$ such that $\chi \mid \otimes \mu_{o}=s \cdot \mu_{o}$. This in turn happens if and only if there is a character $\xi$ of $S$ such that $\chi \otimes \mu=\xi \otimes \mu$. 
But, $\widehat{S}=\widehat{S}_{\mu}$. Hence, there is an $s \in M^{\prime}$ such that $\chi \mid \otimes \mu_{o}=s \cdot \mu_{o}$ if and only if $\chi \in \widehat{R}_{\mu}$.

On the other hand, $\chi \mid$ in $\widehat{V}$ implies $\left.\left(\chi \mid \otimes \mu_{o}\right)\right|_{M_{T}}=\left.\mu_{o}\right|_{M_{T}}=\operatorname{sum}$ of $\left(\delta_{o}\right)$ 's , for $\left(M_{T}^{\prime}\right)_{\delta_{o}}=M_{T}^{\prime}$. Therefore,

$\chi \in \widehat{R}_{\mu} \Longleftrightarrow$ there is an element $s$ in $M_{\delta_{o}}^{\prime}$ such that $\chi \mid \otimes \mu_{o}=s \cdot \mu_{o}$.

Define $\varphi: \widehat{R}_{\mu} \rightarrow M_{\delta_{o}}^{\prime} / M_{T}^{\prime}$ by $\chi \mapsto \bar{s}$. In view of the fact that $M_{T}^{\prime}=M_{\mu_{o}}^{\prime}$ and $\widehat{V}_{\mu_{o}}=1, \varphi$ is well-defined and an isomorphism. This proves (i).

(ii) Second, we note the isomorphism $\widehat{S} \cong \widehat{U}$ is given by restricting characters. Here for any $\chi \in \widehat{S}$ we write $\chi \mid$ for the corresponding character in $\widehat{U}$. Since $\widehat{S}=\widehat{S}_{\mu}$, for all character $\chi \mid$ in $\widehat{U}$ there is $s \in M^{\prime}$ such that $\chi \mid \otimes \delta=s \cdot \delta$. As in (i), we construct a group homomorphism $\psi: \widehat{U} \rightarrow M^{\prime} / M_{\delta}^{\prime}$ by $\chi \mapsto \bar{s}$. Clearly, $\operatorname{ker}(\psi)=\widehat{U}_{\delta}$. We want to prove that $\psi$ is surjective. To do this, note that if $x$ in $M^{\prime}, x=y \cdot m$ for some $y$ in $M_{T}^{\prime}$ and $m$ in $M$, since $M^{\prime}=M_{T}^{\prime} M$. Therefore, $x \cdot \delta=y \cdot \delta$. But $\left(M_{T}^{\prime}\right)_{\delta_{o}}=M_{T}^{\prime}$ implies that $\left.y \cdot \delta\right|_{M_{T}}$ contains $\delta_{o}$. Thus, there exists $\chi \in \widehat{U}$ such that $x \cdot \delta=y \cdot \delta=\chi \otimes \delta$. The sublemma holds.

Now, we prove Lemma (4.3.2) (b). From the proof of (a), consider again the restriction to $G^{\#}$ :

$$
\left.X_{G}(\delta \otimes 1)\right|_{G^{*}}=m\left(\delta_{o}, \delta\right) \sum_{w \in M / M_{\delta_{o}}} X_{G^{*}}\left(w \cdot \delta_{o} \otimes 1\right) .
$$

Choose representatives $\left\{r_{1}, r_{2}, \cdots, r_{m}\right\}$ in $M$ of the classes of $M / M_{\delta_{o}}$; hence, $m=\left|M / M_{\delta_{o}}\right|$. From ([Green], Chapter 2), we can decompose $X_{G^{*}}\left(r_{j} \cdot \delta_{o} \otimes 1\right)$ as the direct sum $\sum_{\chi \in \hat{V}} I_{\chi \cdot r_{j} \cdot \mu_{o}}$, where $I_{\chi \cdot r_{j} \cdot \mu_{o}}$ is the unique irreducible $\left(\mathfrak{g}, T^{c}\right)$ module appearing in the sum whose unique $T^{c}$-fine representation is $\chi \cdot r_{j} \cdot \mu_{o}$ (for any $j=1,2, \cdots m$ ).

As an application of statement (a), we can write $A(\delta)=\left\{\chi_{1} \cdot \mu, \chi_{2} \cdot \mu, \cdots, \chi_{d}\right.$. $\mu\}$ for representatives $\left\{\chi_{1}, \chi_{2}, \cdots, \chi_{d}\right\}$ in $\widehat{R}$ of the classes of $\widehat{R} / \widehat{R_{\mu}}$; therefore $d=\left|\widehat{R} / \widehat{R_{\mu}}\right|$. Again, for any $\chi \in \widehat{R}$ we write $\chi \mid$ for the corresponding character in $\widehat{V}$. Then

$$
\sum_{j=1}^{m} X_{G^{*}}\left(r_{j} \cdot \delta_{o} \otimes 1\right)=\sum_{j=1}^{m} \sum_{\chi \in \hat{R}} I_{\chi \mid \cdot r_{j} \cdot \mu_{o}}=\sum_{j=1}^{m} \sum_{k=1}^{d} \sum_{\xi \in \hat{R}_{\mu}} I_{\chi_{k}|\cdot \xi| \cdot r_{j} \cdot \mu_{o}} .
$$

Sublemma (i) implies $\sum_{j=1}^{m} \sum_{\xi \in \hat{R}_{\mu}} I_{\chi_{k}|\cdot \xi| \cdot r_{j} \cdot \mu_{o}}=\sum_{s \in M / M_{T}} I_{\chi_{k} \mid \cdot s \cdot \mu_{o}}$ for any index $k=1,2, \cdots, d$. This is a $(\mathfrak{g}, K)$-irreducible submodule of $X_{G}(\delta \otimes 1)$ containing the $K$-fine representation $\chi_{k} \cdot \mu$. It appears with multiplicity $m\left(\delta_{o}, \delta\right)=$ $m(\delta, \mu)$. The lemma follows.

The next step is a generalization of the previous lemma.

Lemma 4.3.3. Suppose $G$ is quasisplit and $\delta \in \widehat{M}$ is fine. Then we obtain:

(a) $\widehat{R_{\delta}}$ acts on $A(\delta)$ transitively.

(b) $X_{G}(\delta \otimes 1)=m(\delta, \mu) \sum_{\mu \in A(\delta)} I_{\mu}$ where $I_{\mu}$ is an irreducible admissible $(\mathfrak{g}, K)$-module with unique minimal $K$-type $\mu$. Moreover, $\mu \cong \mu^{\prime}$ in $\widehat{K}$ if 
and only if $I_{\mu}$ and $I_{\mu^{\prime}}$ are equivalent $(\mathfrak{g}, K)$-modules. $I_{\mu}$ appears in the sum $m(\delta, \mu)$-times.

Proof. Recall the definition of $\bar{\Delta}_{s}$ (Proposition (2.30)). Since the nilpotent part of the minimal parabolic subgroup $P$ is not important to obtain $\operatorname{Ind}_{P}^{G}(\delta \otimes 1)$ in view of (4.1.16), we can assume that the positive system $\bar{\Delta}_{+}$is the one corresponding to $P$ as in (4.1.9).

Let $\bar{\Delta}_{o}$ be the set of simple roots of $\bar{\Delta}_{+}$. With $E=\bar{\Delta}_{o} \cap \bar{\Delta}_{S}$, consider the associated $p$-pair $\left(P_{E}, A_{E}\right)$ and the Langlands decomposition $P_{E}=M_{E} A_{E} N_{E}$. Now, we observe the pair $\left(M_{E}, \delta\right)$ satisfies the assumptions in example (4.3.1). Applying induction by stages (Proposition (4.1.14)), we obtain

$$
(*) \operatorname{Ind}_{P}^{G}(\delta \otimes 1)=\operatorname{Ind}_{P_{E}}^{G}\left(\operatorname{Ind}_{P \cap M_{E}}^{M_{E}}(\delta \otimes 1) \otimes 1\right)=m(\delta, \eta) \sum_{\eta \in A^{E}(\delta)} \operatorname{Ind}_{P_{E}}^{G}\left(I_{\eta} \otimes 1\right)
$$

The last part follows from Lemma (4.3.2) (use the bijection in Theorem (4.1.3) (a) to identify the subrepresentations of $\operatorname{Ind}_{P \cap M_{E}}^{M_{E}}(\delta \otimes 1)$ with their corresponding Harish-Chandra modules), where $K_{E}=K \cap M_{E}$, and $A^{E}(\delta)$ is by Definition (2.24) (a) a set of $K_{E}$-fine representations. Write $A_{d}^{E}(\delta)$ for the $\delta$-primary part of elements of $A^{E}(\delta)$, as in definition (2.24) (c).

Write $M_{E}^{\prime}=M^{\prime} \cap M_{E}$. Then $\left(M_{E}^{\prime}\right)_{\delta}=M_{\delta}^{\prime} \cap M_{E}$. Thus, $R_{\delta}^{E}=\left(M_{E}^{\prime}\right)_{\delta} / M$ is the associated $R$-group. We have $M_{\delta}^{\prime}=\left(M_{E}^{\prime}\right)_{\delta}\left(M_{\delta}^{\prime}\right)^{o}$ and $M=\left(M_{E}^{\prime}\right)_{\delta} \cap\left(M_{\delta}^{\prime}\right)^{o}$ by Proposition (2.32) (a) and Definition (2.28). Hence,

$$
R_{\delta}=M_{\delta}^{\prime} /\left(M_{\delta}^{\prime}\right)^{o} \cong\left(M_{E}^{\prime}\right)_{\delta} / M=R_{\delta}^{E} \cong(\mathbb{Z} / 2 \mathbb{Z})^{n}
$$

Use the result $\operatorname{Hom}_{M}\left(\mu_{\delta}, \mu_{\delta}^{\prime}\right)=\operatorname{Hom}_{\left(M_{\delta}^{\prime}\right)^{\circ}}\left(\mu_{\delta}, \mu_{\delta}^{\prime}\right)$ from incise (b) in the proof of Proposition (3.2) to deduce that for $\mu_{\delta}$ and $\mu_{\delta}^{\prime}$ in $A_{d}(\delta)$,

$$
\operatorname{Hom}_{\left(M_{E}^{\prime}\right)_{\delta}}\left(\mu_{\delta}, \mu_{\delta}^{\prime}\right)=\operatorname{Hom}_{M_{\delta}^{\prime}}\left(\mu_{\delta}, \mu_{\delta}^{\prime}\right) \text {. }
$$

This together with Corollary (2.22) implies that for all $\mu \in A(\delta), \quad \eta:=\left.\mu\right|_{K_{E}}$ is an irreducible element in $A^{E}(\delta)$. The isomorphism $\widehat{R_{\delta}} \cong \widehat{R_{\delta}^{E}}$ is defined by restricting characters of $\widehat{R_{\delta}}$. We make use of the $\delta$-primary part. An element $\mu_{\delta}$ in $A_{d}(\delta)$ restricted $\left(M_{\delta}^{\prime}\right)^{o}$ is an irreducible element $\eta_{\delta}$ belonging to $A_{d}^{E}(\delta)$. Another implication of Lemma (4.3.2) is $\widehat{R_{\delta}^{E}} \cdot \eta_{\delta}=A_{d}^{E}(\delta)$.

We build up the following function: $\psi: \widehat{R_{\delta}^{1}} \cdot \mu_{\delta} \rightarrow A_{d}^{E}(\delta)$ by $\chi \otimes \mu_{\delta} \mapsto \chi \mid \otimes \eta_{\delta}$. Note $\chi \cdot \mu_{\delta}=\mu_{\delta}$ if and only if $\chi \mid \cdot \eta_{\delta}=\eta_{\delta}$. Thus, $\psi$ is a bijection. We conclude that $\left|A_{d}^{E}(\delta)\right|=\left|\widehat{R_{\delta}^{1}}\right|$ and $m(\delta, \eta)=\left|\widehat{R_{\delta}^{o}}\right|^{\frac{1}{2}}=m(\delta, \mu)$ by Lemma (3.8).

On the right-hand side of $\left(^{*}\right)$ above we have at least $m(\delta, \mu)\left|\widehat{R_{\delta}^{1}}\right| K$-fine representations while on the left we have exactly $m(\delta, \mu)|A(\delta)| K$-fine representations.

By Proposition (3.2), $|A(\delta)|=\left|A_{d}(\delta)\right| \leq\left|\widehat{R_{\delta}^{1}}\right|$. Thus, $\left|A_{d}(\delta)\right|=\left|\widehat{R_{\delta}^{1}}\right|$.

After Proposition (3.2), $\left|A_{d}(\delta)\right|=\left|\widehat{R_{\delta}^{1}}\right|$ implies that $\widehat{R_{\delta}}$ acts transitively on $A_{d}(\delta)$. We extend this action on $A(\delta)$ by the bijection referred to in (2.25). This means that for any $\chi \in \widehat{R_{\delta}}$ and any $\mu \in A(\delta)$, the product $\chi \cdot \mu$ is by definition the $K$-fine representation such that its $\delta$-primary part is $\chi \cdot \mu_{\delta}$. Thus, the stabilizer $\left(\widehat{R_{\delta}}\right)_{\mu}$ is $\left(\widehat{R_{\delta}}\right)_{\mu_{\delta}}$. This proves $(\mathrm{a})$.

To prove Lemma (4.3.3) (b), we note that $\psi$ above gives rise to the bijection $\Psi: A(\delta) \rightarrow A^{E}(\delta)$ given by the restriction $\mu \mapsto \eta:=\left.\mu\right|_{K_{E}}$. Thus, for all $\eta$ in 
$\left.{ }^{*}\right)$, we observe applying the bijection $\Psi$ that $\operatorname{Ind}_{P_{E}}^{G}\left(I_{\eta} \otimes 1\right)$ is irreducible. $I_{\mu}=$ $\operatorname{Ind}_{P_{E}}^{G}\left(I_{\eta} \otimes 1\right)$ is the unique irreducible submodule that appears in $\operatorname{Ind}_{P}^{G}(\delta \otimes 1)$ containing the $K$-fine representation $\mu=\Psi^{-1}(\eta)$. The lemma follows.

\section{LANGLANDS SUBREPRESENTATIONS AND CLASSIFICATION OF TEMPERED REPRESENTATIONS}

5.1 Limit characters and cohomological induction. The Langlands classification is stated in [Green] in two different ways: using real parabolic induction (as in the introduction), on one hand, and by Vogan-Zuckerman theory of cohomological induction, on the other hand. This result is based on the realization of any standard representation (or rather its Harish-Chandra module which, by Theorem (4.1.7) (a), is equivalent) through cohomological induction. Although the groups considered there are linear, the result and procedure can be extended for groups in the Harish-Chandra class without any change. A standard representation is parametrized by a regular character or equivalently by $\theta$-stable data depending on whether we make use of real parabolic induction or cohomological inducton. However, having in mind our task of decomposing Langlands subrepresentations we are looking for more general type of characters to determine the indecomosable constituents. The following definition comes from [V-II] and [A-B-V].

Let $H=T A$ be a $\theta$-stable Cartan subgroup of $G$, and let $M A=G^{A}$ be the Langlands decomposition of the centralizer of $A$ in $G$. Write $\mathfrak{m}_{o}=\operatorname{Lie}(M)$ and $\mathfrak{t}_{o}=\operatorname{Lie}(T)$.

Definition 5.1.1. A set $(H, \underline{\gamma})=\left(H, \Gamma, \bar{\gamma}, \Delta_{i m}^{+}\right)$is called a pseudo-character for $G$ if it satisfies the following three conditions.

(a) $\Gamma$ is an irreducible representation of $H$ and $\Delta_{i m}^{+}$is a positive system for $\Delta(\mathfrak{m}, \mathfrak{t})$.

(b) $\bar{\gamma} \in \mathfrak{h}^{*}$ is such that for all $\alpha \in \Delta_{i m}^{+},\langle\alpha, \bar{\gamma}\rangle$ is a non-negative real number.

(c) $d \Gamma=\bar{\gamma}+\rho_{\mathfrak{m}}-2 \rho_{\mathfrak{m} \cap \mathfrak{k}}\left(\right.$ where $\rho_{\mathfrak{m}}=\rho\left(\Delta_{i m}^{+}\right)$and $\rho_{\mathfrak{m} \cap \mathfrak{k}}=\rho\left(\Delta_{i m}^{+}\right.$, compact $)$.

Here, $\Delta_{i m, \text { compact }}^{+}$denotes the set of compact roots in $\Delta_{i m}^{+}$. On the other hand, $\Delta_{i m}^{+}$is not superfluous in Definition (5.1.1). However, it is determined by requiring in condition (b) strictly positive instead of non-negative. We have another definition.

Definition 5.1.2. Let $\left(H, \Gamma, \bar{\gamma}, \Delta_{i m}^{+}\right)$be a pseudo-character for $G$.

(a) It is called a regular character if we require that for all $\alpha \in \Delta_{i m}^{+},\langle\alpha, \bar{\gamma}\rangle$ is a strictly positive real number.

(b) It is called a limit character if we require that for any simple compact root $\alpha \in \Delta_{i m}^{+},\langle\alpha, \bar{\gamma}\rangle$ is a strictly positive real number.

(5.1.3) Let $\mathfrak{g}=\mathfrak{k}+\mathfrak{p}$ be a Cartan decomposition of $\operatorname{Lie}(G) \otimes \mathbb{C}$. Similarly to definition (6.5.1) in [Green], we construct the set of data (q, $H, \Gamma_{1}, \bar{\gamma}_{1}$, $\left.\Delta^{+}(\mathfrak{m} \cap \mathfrak{l})\right)$ based on a pseudo-character $(H, \underline{\gamma})=\left(H, \Gamma, \bar{\gamma}, \Delta_{i m}^{+}\right)$for $G$ as follows:

(a) $\mathfrak{q}=\mathfrak{l}+\mathfrak{u}$ is a $\theta$-stable parabolic subalgebra of $\mathfrak{g}$ associated to $\left.\bar{\gamma}\right|_{\mathfrak{t}}$ (Definition (4.2.5)).

(b) $\Gamma_{1} \in \widehat{H}$ is determined by $\left.\Gamma_{1}\right|_{A}=\left.\Gamma\right|_{A}$ and $\left.\Gamma_{1}\right|_{T}=\left.\left.\Gamma\right|_{T} \otimes\left(\Lambda^{\operatorname{dim}(\mathfrak{u} \cap \mathfrak{p})} \mathfrak{u} \cap \mathfrak{p}\right)^{*}\right|_{T}$. 
(c) $\bar{\gamma}_{1}$ is in $\mathfrak{h}^{*}$ defined by $\bar{\gamma}_{1}:=\bar{\gamma}-\rho(\mathfrak{u})$.

(d) $\Delta^{+}(\mathfrak{m} \cap \mathfrak{l})$ is the intersection $\Delta_{i m}^{+} \cap \Delta(\mathfrak{m} \cap \mathfrak{l}, \mathfrak{t})$.

Remark 5.1.4. Consider a set of data $\left(\mathfrak{q}, H, \Gamma_{1}, \bar{\gamma}_{1}, \Delta^{+}(\mathfrak{m} \cap \mathfrak{l})\right)$ as in (5.1.3) based on the pseudo-character $(H, \gamma)=\left(H, \Gamma, \bar{\gamma}, \Delta_{i m}^{+}\right)$for $G$. Let $L$ be the normalizer of $q$. Thus, $H$ is a $\theta$-stable Cartan subalgebra of $L$. Fix a system of positive roots $\Delta^{+}$for $\Delta(\mathfrak{g}, \mathfrak{h})$ such that $\left(\Delta_{i m}^{+} \cup \Delta(\mathfrak{u}, \mathfrak{h})\right) \subseteq \Delta^{+}$. Consider also the positive system $\Delta^{+}(\mathfrak{l}, \mathfrak{h})$ determined by $\Delta^{+} \cap \Delta(\mathfrak{l}, \mathfrak{h})$. In Notation (1.2), write $\rho=\rho\left(\Delta^{+}\right), \rho_{c}=\rho\left(\Delta^{+} \cap \Delta(\mathfrak{k}, \mathfrak{t})\right), \rho_{l x}=\rho\left(\Delta^{+}(\mathfrak{l}, \mathfrak{h})\right), \rho_{\mathfrak{l} \cap \mathfrak{k}}=\rho\left(\Delta^{+} \cap \Delta(\mathfrak{l} \cap \mathfrak{k}, \mathfrak{h})\right)$ and $\rho(\mathfrak{u})=\rho(\Delta(\mathfrak{u}, \mathfrak{h}))$.

(a) We have the following equalities:

$$
\begin{aligned}
d \Gamma_{1} & =\bar{\gamma}-2 \rho(\mathfrak{u} \cap \mathfrak{p})+\rho_{\mathfrak{m}}-2 \rho_{\mathfrak{m} \cap \mathfrak{k}} \\
& =(\bar{\gamma}-\rho(\mathfrak{u}))+(\rho(\mathfrak{u})-2 \rho(\mathfrak{u} \cap \mathfrak{p}))+\rho_{\mathfrak{m}}-2 \rho_{\mathfrak{m} \cap \mathfrak{k}} \\
& =(\bar{\gamma}-\rho(\mathfrak{u}))-\left(\rho-2 \rho_{c}\right)+\rho_{\mathfrak{m}}-2 \rho_{\mathfrak{m} \cap \mathfrak{k}}+\left(\rho_{\mathfrak{l}}-2 \rho_{\mathfrak{l} \cap \mathfrak{k}}\right) .
\end{aligned}
$$

Using [Green], Lemma 5.3.29, we have

$$
\left.\left(\rho-2 \rho_{c}\right)\right|_{\mathfrak{t}}=\rho_{\mathfrak{m}}-2 \rho_{\mathfrak{m} \cap \mathfrak{k}} \quad \text { and }\left.\quad\left(\rho_{\mathfrak{l}}-2 \rho_{\mathfrak{l} \cap \mathfrak{k}}\right)\right|_{\mathfrak{t}}=\rho_{\mathfrak{l} \cap \mathfrak{m}}-2 \rho_{\mathfrak{l} \cap \mathfrak{m} \cap \mathfrak{k}}
$$

We conclude $\left.d \Gamma_{1}\right|_{\mathfrak{t}}=\left(\left.\bar{\gamma}\right|_{\mathfrak{t}}-\rho(\mathfrak{u})\right)+\rho_{\mathfrak{l} \cap \mathfrak{m}}-2 \rho_{\mathfrak{l} \cap \mathfrak{m} \cap \mathfrak{k}}=\left.\bar{\gamma}_{1}\right|_{\mathfrak{t}}+\rho_{\mathfrak{l} \cap \mathfrak{m}}-2 \rho_{\mathfrak{I} \cap \mathfrak{m} \cap \mathfrak{k}}$.

(b) For any simple root $\alpha$ in $\Delta^{+}(\mathfrak{l}, \mathfrak{h})$ we have $\langle\alpha, \rho(\mathfrak{u})\rangle=0$ (consequently, for every root in $\Delta(\mathfrak{l}, \mathfrak{h}))$ since each simple root $\alpha$ in $\Delta^{+}(\mathfrak{l}, \mathfrak{h})$ satisfies

$$
1=\langle\alpha, \rho\rangle=\left\langle\alpha, \rho_{\mathfrak{l}}+\rho(\mathfrak{u})\right\rangle=\left\langle\alpha, \rho_{\mathfrak{l}}\right\rangle+\langle\alpha, \rho(\mathfrak{u})\rangle=1+\langle\alpha, \rho(\mathfrak{u})\rangle .
$$

(c) In view of $(\mathrm{a}),\left(H, \underline{\gamma_{1}}\right)=\left(H, \Gamma_{1}, \overline{\gamma_{1}}, \Delta^{+}(\mathfrak{m} \cap \mathfrak{l})\right)$ is a pseudo-character for $L$.

From (b) we can deduce that for a root $\alpha$ in $\Delta_{i m}^{+}$

$$
\left.\langle\alpha, \bar{\gamma}\rangle=0 \text { if and only if }\left\langle\alpha, \bar{\gamma}_{1}\right\rangle=0 \text { and } \alpha \in \Delta^{+}(\mathfrak{m} \cap \mathfrak{l})\right) .
$$

Therefore, by definition,

$(H, \gamma)$ is a regular character for $G$ if and only if $\left(H, \underline{\gamma_{1}}\right)$ is a regular character for $L$

$(H, \underline{\gamma})$ is a limit character for $G$ if and only if $\left(H, \underline{\gamma_{1}}\right)$ is a limit character for $L$.

Definition 5.1.5. In view of Remark (5.1.4), a set of data (q, $H, \Gamma_{1}, \bar{\gamma}_{1}$, $\left.\Delta^{+}(\mathfrak{m} \cap \mathfrak{l})\right)$ is called a set of $\theta$-stable pseudo-data for $G$ if the following conditions are satisfied:

(a) The Lie algebra $\mathfrak{q}=\mathfrak{l}+\mathfrak{u}$ is a $\theta$-stable parabolic subalgebra of $\mathfrak{g}$.

(b) $\left(H, \Gamma_{1}, \bar{\gamma}_{1}, \Delta^{+}(\mathfrak{m} \cap \mathfrak{l})\right)$ is a pseudo-character data for the normalizer $L=N_{G}(\mathfrak{q})$.

(c) The Lie algebra $q$ is associated to $\left.\bar{\gamma}_{1}\right|_{\mathfrak{t}}$, as in (4.2.6).

If we require additionally in Definition (5.1.5) the set $\left(H, \gamma_{1}\right)=\left(H, \Gamma_{1}, \bar{\gamma}_{1}\right.$, $\left.\Delta^{+}(\mathfrak{m} \cap \mathfrak{l})\right)$ to be a limit character (a regular character) for $L$, we call the set of $\theta$-stable pseudo-data $\left(\mathfrak{q}, H, \underline{\gamma_{1}}\right)=\left(\mathfrak{q}, H, \Gamma_{1}, \bar{\gamma}_{1}, \Delta^{+}(\mathfrak{m} \cap \mathfrak{l})\right)$ a set of $\theta$-stable limit data (respectively a set of $\theta$-stable data) for $G$.

In analogy to [Green], Theorem (6.6.2), we state the following result:

Theorem 5.1.6. In the setting of Remark (5.1.4) and Definition (5.1.5) there is a bijection between pseudo-characters of $G$ and sets of $\theta$-stable pseudo-data for 
$G$, which preserves conjugacy under $K$. Indeed, this correspondence is given by (5.1.3); it associates limit characters (regular characters) of $G$ to sets of $\theta$-stable limit data (respectively to sets of $\theta$-stable data) for $G$.

(5.1.7) For corresponding sets of limit character $(H, \gamma)=\left(H, \Gamma, \bar{\gamma}, \Delta_{i m}^{+}\right)$ for $G$ and $\theta$-stable limit data $\left(\mathfrak{q}, H, \underline{\gamma_{1}}\right)=\left(\mathfrak{q}, H, \Gamma_{1}, \overline{\bar{\gamma}}_{1}, \Delta^{+}(\mathfrak{m} \cap \mathfrak{l})\right)$ under the bijection of Theorem (5.1.6), we can build up $(\mathfrak{g}, K)$-modules. Consider the discrete series or limit of discrete series representation of $M$ (respectively of $L \cap M$ ), denoted $\omega$ (respectively $\omega_{1}$ ) associated to the parameter $\left.\Gamma\right|_{T}$ (respectively $\left.\Gamma_{1}\right|_{T}$ ). Let $P_{1}=M A N$ be a parabolic subgroup of $G$ containing a minimal parabolic subgroup for which the character $\nu:=\Gamma \mid A$ in $\hat{A}$ is negative (Definition (4.1.15)). Thus, $P_{2}:=P_{1} \cap L$ is a parabolic subgroup of $L$ containing a minimal parabolic subgroup for which the character $\nu_{1}:=\left.\Gamma_{1}\right|_{A}$ of $\widehat{A}$ is negative. Then we obtain two induced representations, $\operatorname{Ind}_{P_{1}}^{G}(\omega \otimes \nu)$ of $G$ and $\operatorname{Ind}_{P_{2}}^{L}\left(\omega_{1} \otimes \nu_{1}\right)$ of $L$ with corresponding Harish-Chandra modules $X_{G}(P, \omega \otimes \nu) \in \mathfrak{F}(\mathfrak{g}, K)$ and $X_{L}\left(P_{1}, \omega_{1} \otimes \nu\right) \in \mathfrak{F}(\mathfrak{l}, L \cap K)$ respectively (Proposition (4.1.12) (c)), where $\mathfrak{F}(\mathfrak{g}, K)$ and $\mathfrak{F}(\mathfrak{l}, L \cap K)$ are the categories of admissible representations of finite length associated to the pairs $(\mathfrak{g}, K)$ and $(\mathfrak{l}, L \cap K)$. Now, to apply cohomological induction we need the Zuckerman functors

$$
\mathfrak{R}_{\mathfrak{q}}^{i}(): \mathfrak{F}(\mathfrak{l}, L \cap K) \rightarrow \mathfrak{F}(\mathfrak{g}, K)
$$

It turns out ([Green], Theorem (6.5.9)) that

$$
\mathfrak{R}_{\mathfrak{q}}^{i}\left(X_{L}\left(P_{1}, \omega_{1} \otimes \nu\right)\right)= \begin{cases}X_{G}(P, \omega \otimes \nu) & \text { if } i=S=\operatorname{dim}(\mathfrak{u} \cap \mathfrak{k}), \\ 0 & \text { otherwise. }\end{cases}
$$

Definition-Notation 5.1.8. In the situation (5.1.7), we will refer to $X_{G}(P, \omega \otimes \nu)$ as the representation induced from the limit character $(H, \underline{\gamma})=(H, \Gamma$, $\left.\bar{\gamma}, \Delta_{i m}^{+}\right)$of $G$. To make explicit the limit character, we will write:

$$
X_{G}(P, \omega \otimes \nu)=X_{G}(H, \underline{\gamma})=\mathfrak{R}_{\mathfrak{q}}^{S}\left(X_{L}\left(P_{1}, \omega_{1} \otimes \nu\right)\right)=\mathfrak{R}_{\mathfrak{q}}^{S}\left(X_{L}\left(H, \underline{\gamma_{1}}\right)\right) .
$$

We will also make use of the notation

$$
\boldsymbol{\Theta}^{G}(H, \underline{\gamma})=\Theta^{G}\left(H, \underline{\gamma}, \Delta_{i m}^{+}\right)=\Theta^{G}\left(H, \Gamma, \bar{\gamma}, \Delta_{i m}^{+}\right)
$$

for the global character attached to $X_{G}(H, \underline{\gamma})$.

Formally we can induce a representation for $G$ (possibly zero) from a pseudocharacter $(H, \underline{\gamma})=\left(H, \Gamma, \bar{\gamma}, \Delta_{i m}^{+}\right)$. Consider again a parabolic subgroup $P_{1}=$ $M A N$ containing a minimal parabolic subgroup negative for $\left.\Gamma_{1}\right|_{A}$. We associate to $\left.\Gamma\right|_{T}$ a character $\Theta_{M}$ for $M$ in the sense of Hecht and Schmid ([V-II], Theorem (4.4)). $\operatorname{Ind}_{P_{1}}^{G}\left(\left.\Theta_{M} \otimes \Gamma\right|_{A} \otimes 1\right)$ is a representation of $G$. This construction coincides with the previous one for limit characters. Thus, we write also $\boldsymbol{\Theta}^{G}(H, \underline{\gamma})$ for the corresponding global character.

Remark 5.1.9. What we are calling a pseudo-character in Definition (5.1.2) agrees with the definition of pseudo-character in [V-II], but this is called a limit character in the book [A-B-V]. With our Definition (5.1.2) the representation induced from a pseudo-character is non-zero if and only if it is a limit character; this result can be found in [A-B-V], Proposition 11.9.

The reason to include pseudo-characters in this subsection is to avoid handling cases in the formulation of the Schmid identities that we discuss next. 
(5.1.10) Let $\left(H, \Gamma, \bar{\gamma}, \Delta_{i m}^{+}\right)$be a pseudo-character for $G$ (Definition (5.1.2)). For a simple non-compact root $\beta \in \Delta_{i m}^{+}$, denote $\tilde{\beta}$ for a real root corresponding to $\beta$ under the Cayley transform $C_{\beta}()$. Let $H^{\beta}=T^{\beta} A^{\beta}$ be the $\theta$-stable Cartan subgroup obtained by the application of $C_{\beta}()$ to $H$. Recall the vector $Z_{\tilde{\beta}}$ from Lemma (2.6). Then write $Z_{\beta}$ for the vector $C_{\beta}\left(Z_{\tilde{\beta}}\right)$. Hence if $\mathfrak{h}_{o}=\mathfrak{t}_{o}+\mathfrak{a}_{o}$ and $\mathfrak{h}_{o}^{\beta}=\mathfrak{t}_{o}^{\beta}+\mathfrak{a}_{o}^{\beta}$, we have

$$
\mathfrak{t}_{o}=\mathfrak{t}_{o}^{\beta}+\mathbb{R} Z_{\tilde{\beta}} \quad \text { and } \quad \mathfrak{a}_{o}^{\beta}=\mathfrak{a}_{o}+\mathbb{R} Z_{\beta} .
$$

Define $\bar{\gamma}^{\beta} \in\left(\mathfrak{h}^{\beta}\right)^{*}$ so that

$$
\left.\bar{\gamma}^{\beta}\right|_{\mathfrak{t}^{\beta}}=\left.\bar{\gamma}\right|_{\mathfrak{t}^{\beta}},\left.\quad \bar{\gamma}^{\beta}\right|_{\mathfrak{a}}=\left.\bar{\gamma}\right|_{\mathfrak{a}} \quad \text { and }\left\langle\bar{\gamma}^{\beta}, Z_{\beta}\right\rangle=\left\langle\bar{\gamma}^{\beta}, Z_{\tilde{\beta}}\right\rangle .
$$

The group $T_{1}^{\beta}=T^{\beta} \cap T$, as explained in [Green], Lemma (8.3.5), has the following properties $T_{1}^{\beta} T_{o}=T$ and either $T^{\beta} / T_{1}^{\beta} \cong \mathbb{Z} / 2 \mathbb{Z}$ or $T_{1}^{\beta}=T^{\beta}$. Thus, $\left.\Gamma\right|_{T_{1}^{\beta}}$ is an irreducible character of $T_{1}^{\beta}$. Similarly, the character $\Gamma_{1}^{\beta}$ of $H_{1}^{\beta}=T_{1}^{\beta} A^{\beta}$, defined by

$$
\left.\Gamma_{1}^{\beta}\right|_{T_{1}^{\beta}}=\left.\Gamma\right|_{T_{1}^{\beta}} \text { and }\left.\Gamma_{1}^{\beta}\right|_{A^{\beta}}=\exp \left(\left.\bar{\gamma}^{\beta}\right|_{\mathfrak{a}_{o}^{\beta}}\right)
$$

is irreducible. Hence, we have the following decomposition of irreducible $H^{\beta}$ modules (Lemma (3.8)), for $H^{\beta} / H_{1}^{\beta}$ is isomorphic either to the trivial group or to $\mathbb{Z} / 2 \mathbb{Z}$ :

$$
\operatorname{Ind}_{H_{1}^{\beta}}^{H^{\beta}}\left(\Gamma_{1}^{\beta}\right)=\Gamma^{\beta} \text { or } \Gamma_{+}^{\beta}+\Gamma_{-}^{\beta} .
$$

( $\operatorname{Ind}_{H_{1}^{\beta}}^{H^{\beta}}\left(\Gamma_{1}^{\beta}\right)$ is reducible if and only if $H^{\beta} / H_{1}^{\beta} \cong \mathbb{Z} / 2 \mathbb{Z}$ and for any $x$ in $H^{\beta}$, $x \cdot \Gamma_{1}^{\beta}=\Gamma_{1}^{\beta}$.) Now for the root system $\left\{\left.\alpha\right|_{\mathfrak{t}^{\beta}} \mid \alpha \in \Delta(m x, \mathfrak{t})\right.$ and $\left.\langle\alpha, \beta\rangle=0\right\}$, denote by $\Delta_{i m, \beta}^{+}$the set of positive roots which is determined by $\Delta_{i m}^{+}$. With all the ingredients, we form the sets

$$
\left(H^{\beta}, \underline{\gamma}^{\beta}\right)=\left(H^{\beta}, \Gamma^{\beta}, \bar{\gamma}^{\beta}, \Delta_{i m, \beta}^{+}\right) \text {or }\left(H^{\beta}, \underline{\gamma}_{ \pm}^{\beta}\right)=\left(H^{\beta}, \Gamma_{ \pm}^{\beta}, \bar{\gamma}^{\beta}, \Delta_{i m, \beta}^{+}\right)
$$

according to the previous decomposition. Therefore, we have obtained new pseudo-characters; either $\left(H^{\beta}, \underline{\gamma}^{\beta}\right)$ or $\left(H^{\beta}, \underline{\gamma}_{ \pm}^{\beta}\right)$. It is clear that the characters produced from limit characters are limit characters.

Theorem 5.1.11 ([V-II], Theorem (4.4), Schmid identities). In the situation (5.1.10) suppose $\left(H, \Gamma, \bar{\gamma}, \Delta_{i m}^{+}\right)$is a pseudo-character for $G$. Then we have for a simple non-compact root $\beta$ in $\Delta_{i m}^{+}$with reflexion $s_{\beta}$, and for the pseudo$\operatorname{character}(H, \underline{\tilde{\gamma}})=\left(H, \Gamma-\beta, \bar{\gamma}, s_{\beta}\left(\Delta_{i m}^{+}\right)\right)$, .

$$
\Theta^{G}(H, \underline{\gamma})+\Theta^{G}(H, \underline{\tilde{\gamma}})=\Theta^{G}\left(H^{\beta}, \underline{\gamma}^{\beta}\right) \text { or } \Theta^{G}\left(H^{\beta}, \underline{\gamma}_{+}^{\beta}\right)+\Theta^{G}\left(H^{\beta}, \underline{\gamma}_{-}^{\beta}\right)
$$

according to $(H, \underline{\gamma})$ gives rise either to $\left(H^{\beta}, \underline{\gamma}^{\beta}\right)$ or to $\left(H^{\beta}, \underline{\gamma}_{ \pm}^{\beta}\right)$.

Remark 5.1.12. We can ask when a global character $\Theta^{G}\left(H^{\prime}, \gamma^{\prime}\right)$ attached to the limit character $\left(H^{\prime}, \underline{\gamma^{\prime}}\right)$ appears on the right-hand side of some Schmid identity.

In other words, we are asking when there is a global character $\Theta^{G}(H, \underline{\gamma})$ attached to a limit character $\left(H, \Gamma, \bar{\gamma}, \Delta_{i m}^{+}\right)$appearing on the left of a Schmid 
identity while $\Theta^{G}\left(H^{\prime}, \underline{\gamma^{\prime}}\right)$ appears on the right. A necessary condition is that $\underline{\gamma^{\prime}}=\underline{\gamma}^{\beta}, \underline{\gamma}_{+}^{\beta}$ or $\underline{\gamma}_{-}^{\beta}$ for a certain non-compact root $\beta \in \Delta_{i m}^{+}$.

Suppose that $\alpha \in \Delta(\mathfrak{g}, \mathfrak{h})$ is real and $\left\langle\alpha, \bar{\gamma}^{\prime}\right\rangle=0$. To find $\left(H, \Gamma, \bar{\gamma}, \Delta_{i m}^{+}\right)$, we need the following compatibility condition ([S-V], Chapter V), called a parity condition for $\left(H^{\prime}, \underline{\gamma^{\prime}}\right)$ :

$$
\Gamma\left(m_{\alpha}\right)=(-1)^{n} I \text { where } n=\left\langle\beta, \rho_{\mathfrak{m}}-2 \rho_{\mathrm{m} \cap \mathfrak{k}}\right\rangle
$$

(with the notation developed in (5.1.10); $\alpha=\tilde{\beta}$ ). In fact, this gives us all we need to determine the irreducibility of $\boldsymbol{\Theta}^{G}\left(H^{\prime}, \underline{\gamma^{\prime}}\right)$.

Definition 5.1.13. A limit character $(H, \underline{\gamma})$, Definition (5.1.2), is called a final character if whenever there is a real root $\alpha \in \Delta(\mathfrak{g}, \mathfrak{h})$ orthogonal to $\bar{\gamma}$, it does not satisfy the parity condition (Remark (5.1.12)).

The following result on final characters is proved in [A-B-V], Proposition 11.18 .

Theorem 5.1.14. If $(H, \underline{\gamma})$ is a final character, defined in (5.1.13), then the corresponding induced representation $X_{G}(H, \underline{\gamma}),($ Definition (5.1.8)) has a unique non-zero irreducible subrepresentation. In particular, if $X_{G}(H, \underline{\gamma})$ is a unitary representation induced from a final character, it is irreducible.

(5.1.15) In the setting (5.1.10) we note that if $\left(H, \gamma_{1}\right)$ is a pseudo-character for $L$ and there is a simple non-compact root $\beta$ such $\left\langle\bar{\beta}, \bar{\gamma}_{1}\right\rangle=0$ then we may find either the pseudo-character $\left(H^{\beta}, \underline{\gamma}_{1}^{\beta}\right)$ or the the pseudo-characters $\left(H^{\beta}, \underline{\gamma}_{ \pm}^{\beta}\right)$ for $L$. Therefore, if $\left(\mathfrak{q}, H, \underline{\gamma_{1}}\right)$ is a set of $\theta$-stable pseudo-data corresponding to $(H, \gamma),\left(\mathfrak{q}, H^{\beta}, \underline{\gamma}^{\beta}\right)$ is the set of $\theta$-stable pseudo-data corresponding to $\left(H^{\beta}, \underline{\gamma}^{\beta}\right)$ and $\left.\left(\mathfrak{q}, H^{\beta}, \underline{\gamma}_{ \pm}^{\beta}\right)\right)$ are the sets of $\theta$-stable pseudo-data corresponding to $\left.\left(H^{\beta}, \underline{\gamma}_{ \pm}^{\beta}\right)\right)$.

Proposition 5.1.16. Let $\left(H^{\prime}, \gamma^{\prime}\right)$ be a limit character. Under the bijection of Theorem (5.1.6), let ( $\left.\mathfrak{q}, H^{\prime}, \gamma_{1}^{\prime}\right)$ be the corresponding set of $\theta$-stable limit data. Then we have $\left(H^{\prime}, \underline{\gamma^{\prime}}\right)$ is a final character for $G$ if and only if $\left(H^{\prime}, \underline{\gamma_{1}^{\prime}}\right)$ is a final character for $L$.

Proof. Suppose $\alpha \in \Delta^{+}\left(\mathfrak{g}, \mathfrak{h}^{\prime}\right)$ is a real root such that $\langle\alpha, \bar{\gamma}\rangle=0$. By Remark (5.1.4) (c), there is a root $\left.\alpha \in \Delta^{+}\left(\mathfrak{g}, \mathfrak{h}^{\prime}\right)\right)$ such that $\langle\alpha, \bar{\gamma}\rangle=0$ if and only if $\left\langle\alpha, \bar{\gamma}_{1}^{\prime}\right\rangle=0$ and $\alpha \in \Delta^{+}\left(\mathfrak{l}, \mathfrak{h}^{\prime}\right)$. Let $(H, \underline{\gamma})=\left(H, \Gamma, \bar{\gamma}, \Delta^{+}\right)$be a limit character for $G$ with corresponding set of $\theta$-stable limit data $\left(\mathfrak{q}, H, \gamma_{1}\right)=$ $\left(\mathfrak{q}, H, \Gamma_{1}, \bar{\gamma}_{1}, \Delta^{+}(\mathfrak{m} \cap \mathfrak{l})\right)$. By Remark (5.1.12), there is a Schmid identity for which $\Theta^{G}(H, \underline{\gamma})$ appears on the left-hand side while $\Theta^{G}\left(H^{\prime}, \underline{\gamma}^{\prime}\right)$ appears on the right-hand side if and only if $\Theta^{L}\left(H, \underline{\gamma_{1}}\right)$ appears on the left-hand side of a Schmid identity while $\Theta^{L}\left(H^{\prime}, \underline{\gamma_{1}^{\prime}}\right)$ appears on the right. Recall from (5.1.3) that $\Gamma_{T}=\left.\left.\Gamma_{1}\right|_{T} \otimes\left(\Lambda^{\operatorname{dim}(u \cap \mathfrak{p})} \mathfrak{u} \cap \mathfrak{p}\right)\right|_{T}$. We can deduce from remark (5.1.4) (a) the following identity:

$$
\rho_{\mathfrak{m}}-\left.2 \rho_{\mathfrak{m} \cap \mathfrak{t}}\right|_{\mathfrak{t}}=\rho_{\mathrm{I} \cap \mathfrak{m}}-2 \rho_{\mathrm{I} \cap \mathfrak{m} \cap \mathfrak{t}}+2 \rho(\mathfrak{u} \cap \mathfrak{p})-\left.\rho(\mathfrak{u})\right|_{\mathfrak{t}} .
$$

Let $\beta$ in $\Delta_{i m}^{+}$, as in Theorem (5.1.11), such that $\alpha=\tilde{\beta}$. Since $\langle\beta, \rho(\mathfrak{u})\rangle=0$, write

$$
n=\left\langle\beta, \rho_{\mathfrak{m}}-\left.2 \rho_{\mathfrak{m} \cap \mathfrak{k}}\right|_{\mathfrak{t}}\right\rangle=\left\langle\beta, \rho_{\mathrm{I} \cap \mathfrak{m}}-2 \rho_{\mathrm{I} \cap \mathfrak{m} \cap \mathfrak{k}}\right\rangle+\langle\beta, 2 \rho(\mathfrak{u} \cap \mathfrak{p})\rangle .
$$


Therefore,

$$
\Gamma\left(m_{\alpha}\right)=(-1)^{n} I \text { if and only if } \Gamma_{1}\left(m_{\alpha}\right)=(-1)^{n-\langle\beta, 2 \rho(\mathfrak{u n p})\rangle} I .
$$

Remark (5.1.12) implies that $\alpha$ satisfies the parity condition for the character $\left(H^{\prime}, \underline{\gamma^{\prime}}\right)$ if and only if it does for $\left(H^{\prime}, \underline{\gamma_{1}^{\prime}}\right)$. This proves the proposition.

\subsection{Back to fine representations}

(5.2.1) Assume in this subsection that $G=K A N$ is a quasisplit group (Definition (2.8)). Let $H=M A$ be a maximally split $\theta$-stable Cartan subgroup of $G$. Suppose that $\delta \in \widehat{M}$ is a fine representation (Definition (2.11)), and $\nu$ is a character of $A$. We can form a set of limit character data $(H, \underline{\gamma})=\left(H, \Gamma, \bar{\gamma}, \Delta_{i m}^{+}\right)$ for $G$ by putting

$$
\Gamma=\delta \otimes \nu, \quad \bar{\gamma}=(d \delta, \nu) \quad \text { and } \quad \Delta_{i m}^{+}=\phi .
$$

(5.2.2) Now, in the situation (5.2.1), consider the root systems $\bar{\Delta}$ from Definition (2.3) and $\bar{\Delta}_{\delta}$ from Definition (2.18). It is necessary to compare two different positive systems of roots for $\bar{\Delta}$. One positive system is chosen as in (2.29) and the other one has to do with the presence of the parameter $\nu$, (4.1.15). More explicitly, define the following set of roots:

$$
\begin{aligned}
& \bar{\Delta}_{\nu<0}=\{\alpha \in \bar{\Delta} \mid \text { either }\langle\alpha, \operatorname{Re}(\nu)\rangle<0 \\
& \quad \text { or }(\langle\alpha, \operatorname{Re}(\nu)\rangle=0 \text { and }\langle\alpha, \operatorname{Im}(\nu)\rangle<0)\} .
\end{aligned}
$$

First, choose a positive system $\bar{\Delta}_{\delta}^{+}$for $\bar{\Delta}_{\delta}$ such that it contains the intersection $\bar{\Delta}_{\delta} \cap \bar{\Delta}_{\nu<0}$. Hence, we write the semi-sum of positive roots $\rho_{\delta}=\rho\left(\bar{\Delta}_{\delta}^{+}\right)$(see (1.2)). Our first positive set of roots $\bar{\Delta}^{+o}$ is chosen such that it contains the following set of roots:

$$
\bar{\Delta}_{\rho_{\delta}>0}=\left\{\alpha \in \bar{\Delta} \mid\left\langle\alpha, \rho_{\delta}\right\rangle>0\right\} .
$$

Let $\Phi$ denote the root system defined by $\{\alpha \in \bar{\Delta} \mid\langle\alpha, \nu\rangle=0\}$. The second set of positive roots $\bar{\Delta}^{+1}$ for $\bar{\Delta}$ is elected among those containing the set of roots $\bar{\Delta}_{\nu<0} \cup\left(\Phi \cap \bar{\Delta}_{\rho_{\delta}>0}\right)$.

Similarly, we need to choose two positive systems for root system $\Phi$. Define the set of good roots in $\Phi$ by $\Phi_{\delta}=\Phi \cap \bar{\Delta}_{\delta}$ with positive set of roots $\Phi_{\delta}^{+}=\Phi \cap$ $\bar{\Delta}_{\delta}^{+}$. We denote the semi-sum of positive roots by $\rho_{\Phi_{\delta}}=\rho\left(\Phi_{\delta}^{+}\right)$. We choose one positive system $\Phi^{+_{o}}$ for $\Phi$ such that it contains the set $\left\{\alpha \in \Phi \mid\left\langle\alpha, \rho_{\Phi_{\delta}}\right\rangle>0\right\}$. The other one is defined by $\Phi^{+_{1}}=\Phi \cap \bar{\Delta}^{+1}$. Let $\Pi$ be the set of simple roots in $\bar{\Delta}^{+_{1}}$. Thus, $\Pi_{\Phi}=\Pi \cap \Phi^{+_{1}}$ is the set of simple roots of $\Phi^{+_{1}}$. Recall the set $\bar{\Delta}_{s}^{+}=\left\{\alpha \in \bar{\Delta}^{+_{o}} \mid\left\langle\alpha, \rho_{\delta}\right\rangle=0\right\}$ from Proposition (2.29). In the same way we write $\Phi_{s}^{+}=\left\{\alpha \in \Phi^{+_{o}} \mid\left\langle\alpha, \rho_{\Phi_{\delta}}\right\rangle=0\right\}$.

(5.2.3) In the notation of (5.2.2), we fix a minimal parabolic subgroup $P=$ $M A N$ of $G$ corresponding to the positive root system $\bar{\Delta}^{+_{1}}$. Note that $P$ is negative for $\nu$ (Definition (4.1.15)). Put $D=\Pi_{\Phi}$ and $F=\Pi_{\Phi} \cap \bar{\Delta}_{s}^{+}$. Therefore, $F \subseteq D$ are two sets of simple roots in $\bar{\Delta}^{+1}$. With respect to the positive system of roots $\bar{\Delta}^{+1}$, consider the two $P$-pairs $\left(P_{D}, A_{D}\right)$ and $\left(P_{F}, A_{F}\right)$ with Langlands decompositions $P_{D}=M_{D} A_{D} N_{D}$ and $P_{F}=M_{F} A_{F} N_{F}$ constructed in 
(4.1.10) with $P_{D} \supseteq P_{F} \supseteq P$ and $P_{D} \cap M_{D}$ is a parabolic subgroup of $M_{D}$. Consequently, we have obtained two pairs $\left(\delta, M_{F}\right)$ and $\left(\delta, M_{D}\right)$. The first one satisfies the conditions in Example (4.3.1) and the second one is as in Lemma (4.3.3).

(5.2.4) For any two sets of simple roots $I$ and $J$ in a positive set $\bar{\Delta}^{+}$of the root system $\bar{\Delta}$ such that $I \subseteq J$, we have two $P$-pairs, similarly to (5.2.3), whose corresponding parabolic subgroups possess the Levi parts, $M_{I} A_{I}$ and $M_{J} A_{J}$, satisfying $M_{I} A_{I} \subseteq M_{J} A_{J}$. For any $C \in\{I, J\}$, define $K_{C}=K \cap M_{C}$ and $M_{C}^{\prime}=M^{\prime} \cap M_{C}$ (where $M^{\prime}$ is the normalizer of $A$ in $K$ ). Put $\left(M_{C}^{\prime}\right)_{\delta}=$ $M_{\delta}^{\prime} \cap M_{C}^{\prime}$. Then define in the same way as Definition (2.24),

$A^{C}(\delta)=\left\{\eta \in \widehat{K_{C}} \mid \eta\right.$ is a $K_{C}$-fine representation and $\left.\eta\right|_{M}$ contains $\delta$

as submodule $\}$.

Hence, let $A_{m}^{C}(\delta)$ and $A_{d}^{C}(\delta)$ be the sets of elements in $A^{C}(\delta)$ restricted to $M_{C}^{\prime}$ and $\left(M_{C}^{\prime}\right)_{\delta}$, respectively. Write, $R_{\delta}^{C}=\left(M_{C}^{\prime}\right)_{\delta} / M$. For any element $\eta \in A^{I}(\delta)$ define

$$
A^{J}(\eta)=\left\{\mu \in \widehat{K_{J}} \mid \mu \text { is a } K_{J} \text {-fine representation and }\left.\mu\right|_{K_{I}} \text { contains } \eta\right.
$$

as submodule\}.

We do not write the set $C$ in the previous notation if this is empty.

(5.2.5) Suppose we are in situation (5.2.3). Recall that in the proof of Lemma (4.3.3) we made use of a group $E$. Here for the the pair $\left(\delta, M_{D}\right)$ we define the group $E^{\prime}:=\Phi_{s}^{+}$. It might happen that $F$ is not contained in the set $E^{\prime}$. Now, with respect to $\Phi^{+o}$, we consider the parabolic subgroup of $M_{D}$ corresponding to $E^{\prime}$ having Levi part $M_{E^{\prime}} A_{E^{\prime}}$. One consequence of the proof of Lemma (4.3.3) is that $R_{\delta}^{D}$ is ismorphic to $R_{\delta}^{E^{\prime}}$. We have more.

Lemma 5.2.6. In the situation (5.2.2) and for the set $E^{\prime}$ in (5.2.5), we have

(a) $\left(M_{F}^{\prime}\right)_{\delta}=\left(M_{E^{\prime}}^{\prime}\right)_{\delta}$.

(b) The three $R$-groups $R_{\delta}^{D}, R_{\delta}^{E^{\prime}}$ and $R_{\delta}^{F}$ are isomorphic.

(c) The restriction maps from $A^{D}(\delta)$ to $A^{E^{\prime}}(\delta)$ and to $A^{F}(\delta)$ are bijective.

Proof. First of all, by definition of $D, F$, and $E^{\prime}$, we note that

$\left(M_{D}^{\prime}\right)_{\delta}=\left\{x \in M_{\delta}^{\prime} \mid x \cdot \nu=\nu\right\}, \quad\left(M_{F}^{\prime}\right)_{\delta}=\left\{x \in\left(M_{D}^{\prime}\right)_{\delta} \mid x \cdot \rho_{\Phi_{\delta}}=\rho_{\Phi_{\delta}}\right\}$ and $\left(M_{E^{\prime}}^{\prime}\right)_{\delta}=\left\{x \in\left(M_{D}^{\prime}\right)_{\delta} \mid x \cdot \rho_{\delta}=\rho_{\delta}\right\}$.

Therefore, we have

$$
\begin{aligned}
& \left(M_{F}^{\prime}\right)_{\delta}=\left\{x \in M_{\delta}^{\prime} \mid x \cdot \nu=\nu \text { and } x \cdot \rho_{\Phi_{\delta}}=\rho_{\Phi_{\delta}}\right\} \quad \text { and } \\
& \left(M_{E^{\prime}}^{\prime}\right)_{\delta}=\left\{x \in M_{\delta}^{\prime} \mid x \cdot \nu=\nu \text { and } x \cdot \rho_{\delta}=\rho_{\delta}\right\} .
\end{aligned}
$$

Take $x \in M_{\delta}^{\prime}$ such that $x \cdot \nu=\nu$. Thus, since $\langle x \alpha, \nu\rangle=\langle\alpha, \nu\rangle$ and $x\left(\bar{\Delta}_{\delta}\right)=$ $\bar{\Delta}_{\delta}$, then $x\left(\bar{\Delta}_{\delta}^{+}-\Phi_{\delta}^{+}\right)=\bar{\Delta}_{\delta}^{+}-\Phi_{\delta}^{+}$. Hence, for any $x \in M_{\delta}^{\prime}$ such that $x \cdot \nu=\nu$ we have

$$
x\left(\bar{\Delta}_{\delta}^{+}\right)=\bar{\Delta}_{\delta}^{+} \Longleftrightarrow x\left(\Phi_{\delta}^{+}\right)=\Phi_{\delta}^{+}
$$

Therefore, statement (a) of lemma follows. Statement (a) and proof of Lemma (4.3.3) imply (b), and (c) follows from Corollary (2.19).

(5.2.7) Let $(H, \underline{\gamma})$ be the limit character in (5.2.1). Let $P$ be the minimal parabolic subgroup of $G$ corresponding to $\bar{\Delta}^{+_{1}}$, given in (5.2.3). In (5.2.3), the 
standard representation $\operatorname{Ind}_{P}^{G}(\delta \otimes \nu)$ associated to $(H, \underline{\gamma})$ can be written by applying induction by stages (Proposition (4.1.14)) and by defining $\nu_{D}=\left.\nu\right|_{A_{D}}$ and $\nu_{F}=\left.\nu\right|_{A_{F}}$, as follows:

$$
\begin{aligned}
\operatorname{Ind}_{P}^{G}(\delta \otimes \nu) & =\operatorname{Ind}_{P_{D}}^{G}\left(\operatorname{Ind}_{P_{F} \cap M_{D}}^{M_{D}}\left(\operatorname{Ind}_{P \cap M_{F}}^{M_{F}}(\delta \otimes 1) \otimes 1\right) \otimes \nu_{D}\right) \\
& =\operatorname{Ind}_{P_{F}}^{G}\left(\operatorname{Ind}_{P \cap M_{F}}^{M_{F}}(\delta \otimes 1) \otimes \nu_{F}\right) .
\end{aligned}
$$

As a consequence of Lemma (4.3.3), we have two decompositions:

$$
\begin{aligned}
& \operatorname{Ind}_{P \cap M_{D}}^{M_{D}}(\delta \otimes 1)=m(\delta, \xi) \sum_{\xi \in A^{E^{\prime}}(\delta)} I_{\xi}, \\
& \operatorname{Ind}_{P \cap M_{F}}^{M_{F}}(\delta \otimes 1)
\end{aligned}
$$

Thus, we obtain

$$
\operatorname{Ind}_{P \cap M_{D}}^{M_{D}}(\delta \otimes 1)=m(\delta, \eta) \sum_{\eta \in A^{F}(\delta)} \operatorname{Ind}_{P_{F} \cap M_{D}}^{M_{D}}\left(I_{\eta} \otimes 1\right) .
$$

By Lemma (5.2.6), the restriction map that goes from $A^{E^{\prime}}(\delta)$ to $A^{F}(\delta)$ is bijective, and $m(\delta, \eta)=m(\delta, \xi)$. This implies that for all $\eta \in A^{F}(\delta)$, the representation $\operatorname{Ind}_{P_{F} \cap M_{D}}^{M_{D}}\left(I_{\eta} \otimes 1\right)$ is irreducible. Substituting $(* *)$ in $(*)$ we get the following series of equalities:

$$
\begin{aligned}
\operatorname{Ind}_{P}^{G}(\delta \otimes \nu) & =m(\delta, \eta) \sum_{\eta \in A^{F}(\delta)} \operatorname{Ind}_{P_{D}}^{G}\left(\operatorname{Ind}_{P_{F} \cap M_{D}}^{M_{D}}\left(I_{\eta} \otimes 1\right) \otimes \nu_{D}\right) \\
& =m(\delta, \eta) \sum_{\eta \in A^{F}(\delta)} \operatorname{Ind}_{P_{F}}^{G}\left(I_{\eta} \otimes \nu_{F}\right) .
\end{aligned}
$$

(5.2.8) Suppose $(H, \underline{\gamma})$ is the limit character given in (5.2.1). Consider $F$, the set of simple roots given in (5.2.3). Let $H_{F}=T_{F} A_{F}$ be a $\theta$-stable Cartan subgroup arising from application of Cayley transforms on $H$ with respect to $F$ such that $T_{o} \subseteq T_{F}$ and $A_{F} \subseteq A$. Fix $\eta \in A^{F}(\delta)$. Fix any irreducible $T_{F}$-module $\eta_{o}$ occurring in the restriction $\left.\eta\right|_{T_{F}}$. We define a limit character $\left(H_{F}, \gamma_{F}\right)=\left(H_{F}, \Gamma_{F}, \overline{\gamma_{F}}, \Delta_{F}^{+}\right.$, im $)$for $G$ as follows:

(i) $\Gamma_{F}$ is the irreducible $H_{F}$-module given by $\left.\Gamma_{F}\right|_{T_{F}}=\eta_{o}$ and $\left.\Gamma_{F}\right|_{A_{F}}=$ $\left.\Gamma\right|_{A_{F}}=\nu_{F}$.

(ii) $\overline{\gamma_{F}} \in \mathfrak{h}_{F}^{*}$ is given by $\left.\overline{\gamma_{F}}\right|_{\mathfrak{t}}=d \delta,\left.\overline{\gamma_{F}}\right|_{\mathfrak{t}^{\perp}} \equiv 0$ and $\left.\overline{\gamma_{F}}\right|_{\mathfrak{a}_{F}}=\nu_{F}$; where $\mathfrak{t}_{F}=\mathfrak{t}+\mathfrak{t}^{\perp}$.

(iii) $\Delta_{F, i m}^{+}$is the unique positive system of $\Delta\left(\mathfrak{m}_{F}, \mathfrak{t}_{F}\right)$ such that $d \eta_{o}$ is dominant.

Remark 5.2.9. (a) The construction of $\left(H_{F}, \underline{\gamma_{F}}\right)$ in (5.2.8), up to conjugation, depends on $\eta$ and not on the choice of $\eta_{o}$ (Recall from proof of Lemma (4.3.2) Case 2 that $\left.m\left(\eta_{o}, \eta\right)=1\right)$. (b) From part (***) in (5.2.6) we first note that $X_{G}\left(P_{F}, I_{\eta} \otimes \nu_{F}\right)$, the Harish-Chandra module associated to $\operatorname{Ind}_{P_{F}}^{G}\left(I_{\eta} \otimes \nu_{F}\right)$, is the representation induced from the limit character $\left(H_{F}, \underline{\gamma_{F}}\right)$ as in (5.1.7).

Lemma 5.2.10. Assume the previous notation. Then $X_{G}\left(P_{F}, I_{\eta} \otimes \nu_{F}\right)=$ $X_{G}\left(H_{F}, \gamma_{F}\right)$ has a unique irreducible $(\mathfrak{g}, K)$-submodule $\bar{X}_{G}\left(H_{F}, \underline{\gamma}_{F}\right)$ (Definition (4.1.19)). 
Proof. As explained in (5.2.7), the $M_{D}$-representation $\operatorname{Ind}_{P \cap M_{D}}^{M_{D}}\left(I_{\eta} \otimes 1\right)$ is irreducible. On the other hand, set $B=\left\{\alpha \in \bar{\Delta}^{+1} \mid\langle\alpha, \operatorname{Re}(\nu)\rangle=0\right\}$ and let $\left(P_{B}, A_{B}\right)$ be the corresponding $p$-pair with Langlands decomposition $P_{B}$ $=M_{B} A_{B} N_{B}$ such that $P_{B} \supseteq P_{D}$. Then, applying induction by stages to Ind $_{P_{F}}^{G}\left(I_{\eta} \otimes \nu_{F}\right)$ we obtain

(\#) $\operatorname{Ind}_{P_{D}}^{G}\left(\operatorname{Ind}_{P_{F} \cap M_{D}}^{M_{D}}\left(I_{\eta} \otimes 1\right) \otimes \nu_{D}\right)=\operatorname{Ind}_{P_{B}}^{G}\left(\operatorname{Ind}_{P_{D} \cap M_{B}}^{M_{B}}\left(\operatorname{Ind}_{P_{F} \cap M_{D}}^{M_{D}}\left(I_{\eta} \otimes 1\right) \otimes \nu_{I}\right) \otimes \nu_{R}\right)$ where $\nu_{I}=\left.\nu\right|_{A_{D} \cap M_{B}}, \nu_{R}=\left.\nu\right|_{A_{B}}$.

By a result of Harish-Chandra, given in [H-Ch 2], on the commuting algebra of induced representations, $\operatorname{Ind}_{P_{D} \cap M_{B}}^{M_{B}}\left(\operatorname{Ind}_{P_{F} \cap M_{D}}^{M_{D}}\left(I_{\eta} \otimes 1\right) \otimes \nu_{I}\right)$ is an irreducible tempered representation since no root $\alpha \in \Delta\left(P_{D} \cap M_{B}, A_{D} \cap M_{B}\right)$ (Definition 4.1.10) is orthogonal to $\nu_{I}$. So the right-hand side of (\#) contains a unique irreducible subrepresentation by [W-2], (5.4.1), since no root $\alpha \in \Delta\left(P_{B}, A_{B}\right)$ (Definition (4.1.10)) is orthogonal to $\nu_{R}$. The lemma follows.

Remark 5.2.11. (a) The limit character $\left(H_{F}, \underline{\gamma_{F}}\right)$ constructed in (5.2.8), for some $\eta \in A^{F}(\delta)$, is not necessarily final (Definition (5.1.13)). However, by Lemma (5.2.10), the induced module $X_{G}\left(H_{F}, \gamma_{F}\right)$ is also induced from a final character. This means that exhausting all the real roots orthogonal to $\overline{\gamma_{F}}$ that satisfy the parity condition (5.1.12) and using the Schmid identities, we can pass from $\left(H_{F}, \underline{\gamma_{F}}\right)$ to a final character $\left(H_{\mathfrak{F}}, \gamma_{\mathfrak{F}}\right)$ such that $X_{G}\left(H_{\mathfrak{F}}, \gamma_{\mathfrak{F}}\right)=$ $X_{G}\left(H_{F}, \gamma_{F}\right)$; that is, we do not get more reducibility. (b) The set of minimal $K$-types for $X_{G}\left(H_{F}, \gamma_{F}\right)$ is, by Frobenius reciprocity theorem, the set $A(\eta)$, (Notation (5.2.3)). Each appears with multiplicity $m(\eta, \mu)$ (for any $\mu \in A(\delta)$ ).

(5.2.12) Suppose $\left(\delta, \eta_{o}, \eta\right)$ is as described in (5.2.8). By the proof of Lemma (4.3.3), the $R$-group $\widehat{R^{F}}$ acts on $A^{F}(\delta)$ and, by restriction, on the set

$$
\mathbb{A}=\left\{\gamma_{o} \in \widehat{T_{F}} \mid \gamma_{o} \text { occurs in }\left.\gamma\right|_{T_{F}} \text { for some } \gamma \in A^{F}(\delta)\right\} \text {. }
$$

By the same Lemma (4.3.3), we have $A^{F}(\delta)=\widehat{R_{F}} \cdot \eta$ and $m\left(\eta_{o}, \eta\right)=1$. Therefore,

$$
\widehat{R_{F}} \cdot \eta=\left\{\operatorname{Ind}_{T_{F}}^{K_{F}}\left(\chi \mid \cdot \eta_{o}\right) \mid \chi \in \widehat{R_{F}}\right\} .
$$

Next, we extend the action of the $R$-group $\widehat{R_{F}}$ to a set of characters.

Definition 5.2.13. We define a set of limit characters,

$$
\boldsymbol{\Omega}_{F}:=\left\{\left(H_{F}, \underline{\gamma_{F}}\right) \mid\left(H_{F}, \underline{\gamma_{F}}\right) \text { is built in (5.2.8) for some } \eta \in A^{F}(\delta)\right\} \text {. }
$$

Define an action of $\widehat{R_{F}}$ on $\Omega_{F}$ as follows: for any limit character in $\Omega_{F}$, say $\left(H_{F}, \underline{\gamma_{F}}\right)=\left(H_{F}, \Gamma_{F}, \overline{\gamma_{F}}, \Delta_{F, i m}^{+}\right)$, and any character $\chi \in \widehat{R^{F}}$, we set

$$
\chi \cdot\left(H_{F}, \underline{\gamma_{F}}\right)=\left(H_{F}, \Gamma_{F}^{\prime}, \overline{\gamma_{F}}, \chi \cdot \Delta_{F, i m}^{+}\right)
$$

where $\left.\Gamma_{F}^{\prime}\right|_{T_{F}}=\left.\chi \cdot \Gamma_{F}\right|_{T_{F}}$ and $\left.\Gamma_{F}^{\prime}\right|_{A_{F}}=\nu_{F}$ and $\chi \cdot \Delta_{F}^{+}$,im is the unique positive system such that $d\left(\chi \cdot \Gamma_{F}\right)$ is dominant.

(5.2.14) In view of (5.2.13), the $R$-group $\widehat{R^{F}}$ acts transitively on $\Omega_{F}$. Since the induction of a module $X_{G}\left(H_{F}, \underline{\gamma_{F}}\right)$ depends only on the class of conjugation of $\left(H_{F}, \gamma_{F}\right)$ by $K$, given $\left(H_{F}, \underline{\gamma_{F}}\right) \in \Omega_{F}$ and $\chi \in \widehat{R_{F}}$, we can deduce that $X_{G}\left(\chi \cdot\left(\overline{H_{F}}, \underline{\gamma_{F}}\right)\right)=X_{G}\left(H_{F}, \underline{\gamma_{F}}\right)$ if and only if $\left.\chi \cdot \Gamma_{F}\right|_{T_{F}}=\left.x \cdot \Gamma_{F}\right|_{T_{F}}$ for some $x \in M_{F}^{\prime}$. That is the case if and only if $\chi \cdot \eta=\eta$, where $\eta=\operatorname{Ind}_{T_{F}}^{K_{F}}\left(\left.\Gamma_{F}\right|_{T_{F}}\right)$. 
Definition 5.2.15. For any character $\bar{\chi} \in \widehat{R^{F}} /\left(\widehat{R^{F}}\right)_{\eta}$, let $\bar{\chi} \cdot X_{G}\left(H_{F}, \gamma_{F}\right)$ be the $(\mathfrak{g}, K)$-module $X_{G}\left(\chi \cdot\left(H_{F}, \underline{\gamma_{F}}\right)\right)$. As explained in (5.2.14), this is well-defined (it does not depend on the representative $\chi$ of $\bar{\chi}$ in $\widehat{R^{F}}$ ). By Lemma (5.2.10), let $\bar{X}_{G}\left(H_{F}, \gamma_{F}\right)$ be the unique irreducible submodule $X_{G}\left(H_{F}, \gamma_{F}\right)$. We define, naturally, $\bar{\chi} \cdot \overline{\bar{X}}_{G}\left(H_{F}, \underline{\gamma_{F}}\right)$ to be the unique irreducible $(\mathfrak{g}, K)$-subrepresentation of $\bar{\chi} \cdot X_{G}\left(H_{F}, \underline{\gamma_{F}}\right)$.

We deduce that each indecomosable constituent in the decomposition of $X_{G}(H, \underline{\gamma})$ is induced from some limit character in $\Omega_{F}$. Moreover, we have a transitive action of $\widehat{R^{F}}$ on the set of the representations induced from limit characters in $\Omega_{F}$. More specifically,

Proposition 5.2.16. We continue considering the character $(H, \underline{\gamma})$ defined in (5.2.1). For any limit character $\left(H_{F}, \gamma_{F}\right) \in \Omega_{F}$ constructed for some $\eta \in A^{F}(\delta)$. We have

$$
X_{G}(\delta \otimes \nu)=m(\delta, \eta) \sum_{\bar{\chi} \in \hat{R}^{F} /\left(\hat{R}^{F}\right)_{\eta}} X_{G}\left(\chi \cdot\left(H_{F}, \underline{\gamma_{F}}\right)\right)
$$

based on Definition (5.2.15).

(5.2.17) We want to give the decomposition in Proposition (5.2.16) in terms of the $R$-group $\widehat{R_{\delta}}$ defined in (2.19) and the set of $K$-fine representations $A(\delta)$ defined in (2.24). To this end, assume we are in the setting (5.2.2). Define $E=\bar{\Delta}_{s}^{+}$. Then, from the proof of Lemma (4.3.3), there is a bijection $\psi$ between $A_{d}(\delta)$ and $A_{d}^{E}(\delta)$ (hence, between $A(\delta)$ and $A^{E}(\delta)$ ), and that the restriction map from $\widehat{R_{\delta}}$ to $\widehat{R_{\delta}^{E}}$ is an isomorphism of groups. Thus, $F$ defined in (5.2.3) is contained in $E$. Hence, we write $A^{E}(\eta)=\psi(A(\eta))$ for any $\eta \in A^{F}(\delta)$, as in Notation (5.2.4).

Notation 5.2.18. In the setting of (5.2.2) and (5.2.4) we define, in analogy to lemma (3.8), for any $\eta_{\delta} \in A_{d}^{F}(\delta)$ the sets

$$
\begin{aligned}
& Q(\delta)=\left\{\chi \in \widehat{R_{\delta}}|\chi|_{\left(M_{F}^{\prime}\right)_{\delta}} \cdot \eta_{\delta}=x \cdot \eta_{\delta} \text { for some } x \in\left(M_{E}^{\prime}\right)_{\delta}\right\}, \\
& Q(\delta)_{o}=\left\{\chi \in \widehat{R_{\delta}}|\chi|_{\left(M_{F}^{\prime}\right)_{\delta}} \cdot \eta_{\delta}=\eta_{\delta}\right\} .
\end{aligned}
$$

These definitions do not depend on the element $\eta_{\delta} \in A_{d}^{F}(\delta)$. Similarly, we denote

$$
Q(\delta)^{E}=\left\{\left.\chi\right|_{\left(M_{E}^{\prime}\right)_{\delta}} \mid \chi \in Q(\delta)\right\} \text { and } Q(\delta)_{o}^{E}=\left\{\left.\chi\right|_{\left(M_{E}^{\prime}\right)_{\delta}} \mid \chi \in Q(\delta)_{o}\right\}
$$

(5.2.19) Now, choose $\mu \in A_{m}^{E}(\delta)$ and $\eta \in A_{m}^{F}(\delta)$ such that $\left.\mu\right|_{\left(M_{F}^{\prime}\right)}$ contains $\eta$ as a submodule. We are in the situation of Lemma (3.8) with $\left(\delta, \eta_{\delta}, \mu_{\delta}, M\right.$, $\left.M_{F}^{\prime}, M_{E}^{\prime}\right)$ instead of $(\delta, \eta, \mu, B, C, A)$ appearing there. Hence, $R^{E}, R^{F}$, $Q(\delta)^{E}$ and $Q(\delta)_{o}^{E}$ correspond to $R, S, Q$ and $Q_{o}$, respectively, in the proof of Lemma (3.8). For any element $\chi \in \widehat{R}$ write $\bar{\chi}$ for its class in $\widehat{R} / Q_{o}$ and $\overline{\bar{\chi}}$ for its class in $\widehat{R} / Q$.

Proposition 5.2.20. Recall the definition of $A_{m}^{E}(\eta)$ in (5.2.4). Then,

(a) For any $\chi \in \widehat{R_{E}}$ denote $\chi|=\chi|_{M_{F}^{\prime}}$. Then the following three conditions are equivalent. 
(i) $A_{m}^{E}(\chi \mid \cdot \eta) \cap A_{m}^{E}(\eta) \neq \varnothing$.

(ii) $A_{m}^{E}(\chi \mid \cdot \eta)=A_{m}^{E}(\eta)$.

(iii) $\chi \in Q(\delta)^{E}$.

(b) We adopt the notation in (5.2.19). In view of (a), there is no ambiguity in defining for any $\chi \in \widehat{R^{E}}$ the set $\overline{\bar{\chi}} \cdot A_{m}^{E}(\eta)=A_{m}^{E}(\chi \mid \cdot \eta)$ where $\overline{\bar{\chi}} \in \widehat{R^{E}} / Q(\delta)^{E}$. Moreover, we have the disjoint union

$$
A_{m}^{E}(\delta)=\bigsqcup_{\overline{\bar{\chi}} \in \hat{R}^{E} / Q(\delta)^{E}} \overline{\bar{\chi}} \cdot A_{m}^{E}(\eta) .
$$

(5.2.21) In the same way as in Proposition (5.2.20), we get the decomposition of $A^{E}(\delta)$, which is consequence of Lemma (4.3.3). Replacing the $R$-group, we get by the proof of Lemma (4.3.3),

$$
A(\delta)=\bigsqcup_{\overline{\bar{\chi}} \in \hat{R}_{\delta} / Q(\delta)} \overline{\bar{\chi}} \cdot A(\eta)
$$

where the set $\overline{\bar{\chi}} \cdot A(\eta)$ corresponds to $\overline{\bar{\chi}} \cdot A_{m}^{E}(\eta)$, by definition, under the bijections given from $A(\delta)$ to $A^{E}(\delta)$ and from $A^{E}(\delta)$ to $A_{m}^{E}(\delta)$. We use the isomorphism between the groups $\widehat{R_{\delta}}$ and $\widehat{R_{\delta}^{E}}$.

Now, we have additional isomorphisms $\widehat{R_{\delta}} / Q(\delta)_{o} \cong \widehat{R_{\delta}^{E}} / Q(\delta)_{o} \cong \widehat{R_{\delta}^{F}} /\left(\widehat{R_{\delta}^{F}}\right)_{\eta_{\delta}}$, where the second one is established in the first part of proof of Lemma (3.11). These give rise, by Corollary (3.9), to the isomorphism $\widehat{R_{\delta}} / Q(\delta)_{o} \cong \widehat{R^{F}} /\left(\widehat{R^{F}}\right)_{\eta}$.

Theorem 5.2.22. Consider the limit character $(H, \gamma)$ defined in (5.2.1). For a fixed limit character $\left(H_{F}, \underline{\gamma_{F}}\right)$ in $\Omega_{F}$ defined in (5.2.13), with respect to some $\eta \in A^{F}(\delta)$ :

(a) We obtain the following decompositions (Definitions (4.1.19) and (5.2.15)),

$$
\begin{aligned}
& X_{G}(H, \underline{\gamma})=m(\delta, \eta) \sum_{\bar{\chi} \in \hat{R}_{\delta} / Q(\delta)_{o}} \bar{\chi} \cdot X_{G}\left(H_{F}, \underline{\gamma_{F}}\right), \\
& \bar{X}_{G}(H, \underline{\gamma})=m(\delta, \eta) \sum_{\bar{\chi} \in \hat{R}_{\delta} / Q(\delta)_{o}} \bar{\chi} \cdot \bar{X}_{G}\left(H_{F}, \underline{\gamma_{F}}\right) .
\end{aligned}
$$

(b) The set of minimal $K$-types of $\bar{\chi} \cdot X_{G}\left(H_{F}, \underline{\gamma_{F}}\right)$ is the set $\overline{\bar{\chi}} \cdot A(\eta)$.

(c) $\overline{\bar{\chi}} \cdot A(\eta)=A(\eta)$ if and only if $\overline{\bar{\chi}}=1$.

(d) $\bar{\chi} \cdot X_{G}\left(H_{F}, \gamma_{F}\right) \cong X_{G}\left(H_{F}, \underline{\gamma}_{F}\right)$ if and only if $\bar{\chi}=1$.

(e) The multiplicity of $\bar{\chi} \cdot X_{G}\left(\overline{H_{F}}, \underline{\gamma_{F}}\right)$ in $X_{G}(\delta \otimes \nu)$ is $m(\delta, \eta)$.

(f) $m(\delta, \eta)=2^{m}$ for some $m \in \mathbb{N}$.

Proof. (a) is Proposition (5.2.16) and Lemma (5.2.10). Remark (5.2.11) (b) implies (b). (c) is (5.2.21) and Proposition (5.2.20). (d) follows from Definition (5.2.14) while (e) is obvious. Corollary (2.33) and Lemma (3.8) imply (f). The theorem follows. 


\subsection{In the general case}

(5.3.1) To begin with, fix a regular character $(H, \gamma)$ with corresponding set of $\theta$-stable limit character $\left(\mathfrak{q}, H, \gamma_{1}\right)$ under the bijection in Theorem (5.1.6). By Remark (5.1.4), the character $\left(\bar{H}, \underline{\gamma_{1}}\right)=\left(H, \Gamma_{1}, \bar{\gamma}_{1}, \Delta_{i m}^{+}\right)$is a regular character for $L$. Then, $\delta=\left.\Gamma_{1}\right|_{T}$ is a $T$-fine representation. Write $\nu=\left.\Gamma_{1}\right|_{A}$. Therefore, the character $\left(H, \gamma_{1}\right)$ is as in (5.2.1) for $L$. As in subsection (5.2), we can find a strongly orthogonal set $F \subset \Delta(\mathfrak{l}, \mathfrak{h})$ of real roots. Write $A_{L}^{F}(\delta)$ for the set $A^{F}(\delta)$ there. Hence, for $\eta \in A_{L}^{F}(\delta)$ we construct a limit character $\left(H_{F}, \gamma_{1 F}\right)$ for $L$, as in Notation (5.2.8), with respect to $\left(H, \underline{\gamma_{1}}\right)$. We make use again of Theorem (5.1.6) to get the limit characters $\left(H_{F}, \underline{\left.\gamma_{F}\right)}\right.$ for $G$ corresponding to the set of $\theta$-stable limit data $\left(\mathfrak{q}, H_{F}, \underline{\gamma_{1 F}}\right)$. Hence, we have

$$
\mathfrak{R}_{\mathfrak{q}}^{S}\left(X_{L}\left(H_{F}, \underline{\gamma_{1 F}}\right)\right)=X_{G}\left(H_{F}, \underline{\gamma_{F}}\right)
$$

for any two corresponding characters $\left(H_{F}, \underline{\gamma_{F}}\right)$ and $\left(\mathfrak{q}, H_{F}, \underline{\gamma_{1 F}}\right)$, where $S$ is $\operatorname{dim}(\mathfrak{u} \cap \mathfrak{p})$. Since we want to apply everything that has been said in (5.2) about fine representations for the limit character $\left(H, \underline{\gamma_{1}}\right)$ of $L$ and $\delta=\left.\Gamma_{1}\right|_{T}$, we complicate the notation a little bit. We write $\widehat{R_{\delta}}(L), Q(\delta, L), Q(\delta, L)_{o}$, $A_{L}(\delta), A_{L}(\eta)$ and $A_{L}^{F}(\delta)$ for the corresponding groups $\widehat{R_{\delta}}, Q(\delta), Q(\delta)_{o}$, $A(\delta), A(\eta)$ and $A^{F}(\delta)$ used to decompose the standard representation in (5.2). In particular, Proposition (5.2.20) (b) establishes the decomposition

$$
A_{L}(\delta)=\bigsqcup_{\overline{\bar{\chi}} \in \hat{R}_{\delta} / Q(\delta, L)} \overline{\bar{\chi}} \cdot A_{L}(\eta)
$$

for $\eta \in A_{L}^{F}(\delta)$.

(5.3.2) We consider $A_{L}(\delta)$ for the set of minimal $L \cap K$-types occurring in $X_{L}\left(H, \underline{\gamma_{1}}\right)$. As an application of Corollary (4.2.8), write $A^{K}(\mathfrak{q}, \delta)$ for the $K$ irreducible representations arisen from the set $\mathbb{A}=\left\{\pi \otimes \Lambda^{\operatorname{dim} u \cap \mathfrak{p}} \mathfrak{u} \cap \mathfrak{p} \mid \pi \in\right.$ $\left.A_{L}(\delta)\right\}$ consisting of $L \cap K$-irreducible representations: we use the fact that the $\theta$-stable parabolic subalgebra associated to the Vogan $\lambda$-element $(4.2 .4)$ for any object of $\mathbb{A}$ is exactly $\mathfrak{q}$ ([Green], Lemma (5.3.23)). Write $A^{K}(\eta)$ for the subset of $A^{K}(\mathfrak{q}, \delta)$ that arises from elements of $A^{L}(\delta)$ multiplied by $\Lambda^{\operatorname{dim}(\mathfrak{u} \cap \mathfrak{p})} \mathfrak{u} \cap \mathfrak{p}$, as above. It turns out that $A^{K}(\mathfrak{q}, \delta)$ is the set of minimal $K$-types for the standard representation $X_{G}(H, \gamma)$. This is the statement in [Green], Theorem (6.5.9) (d), omitting the multiplicity-one assertion. Moreover, we can deduce from there that $A^{K}(\eta)$ is the set of minimal $K$-types of $X_{G}\left(H_{F}, \underline{\gamma_{F}}\right)=$ $\mathfrak{R}_{\mathfrak{q}}^{S}\left(X_{L}\left(H_{F}, \underline{\gamma_{1 F}}\right)\right)$ (compare this result with Theorem (5.2.22) (b)).

(5.3.3) From Lemma (5.2.10), the $(\mathfrak{l}, L \cap K)$-module $X_{L}\left(H_{F}, \gamma_{1 F}\right)$ has a unique irreducible submodule. By a similar argument to the proof of Lemma (5.2.10) or by Theorem 6.5.10 (d) in [Green], in terms of cohomological induction, $X_{G}\left(H_{F}, \underline{\gamma_{F}}\right)$ has a unique $(\mathfrak{g}, K)$-irreducible submodule that we denote by $\bar{X}_{G}\left(H_{F}, \underline{\gamma_{F}}\right)$.

Notation 5.3.4. (a) As in Proposition (5.2.20), we adopt the next notation : For any element $\chi$ in $\widehat{R_{\delta}}(L)$, we write $\bar{\chi}$ for its corresponding class in $\widehat{R_{\delta}}(L) / Q(\delta, L)_{o}$ and $\overline{\bar{\chi}}$ for its corresponding class in $\widehat{R_{\delta}}(L) / Q(\delta, L)$. 
(b) In view of (5.3.3), for any $\bar{\chi} \in \widehat{R_{\delta}}(L) / Q(\delta, L)_{o}$, define

$$
\bar{\chi} \cdot X_{G}\left(H_{F}, \underline{\gamma_{F}}\right):=\mathfrak{R}_{\mathfrak{q}}^{S}\left(\bar{\chi} \cdot X_{L}\left(H_{F}, \underline{\gamma_{1 F}}\right)\right)
$$

and $\bar{\chi} \cdot \bar{X}_{G}\left(H_{F}, \underline{\gamma_{F}}\right)$ to be the unique irreducible $(\mathfrak{g}, K)$-submodule of $\bar{\chi}$. $X_{G}\left(H_{F}, \underline{\gamma_{F}}\right)$.

(c) In view of (5.3.2), for any $\overline{\bar{\chi}} \in \widehat{R_{\delta}}(L) / Q(\delta, L)$, define $\overline{\bar{\chi}} \cdot A^{K}(\eta)$ to be the subset of $A^{K}(\mathfrak{q}, \delta)$ corresponding to $\overline{\bar{\chi}} \cdot A_{L}(\eta)$ under the bijection between $A^{K}(\mathfrak{q}, \delta)$ and $A_{L}(\delta)$ established.

Therefore, we have once more a disjoint union

$$
A^{K}(\mathfrak{q}, \delta)=\bigsqcup_{\overline{\bar{\chi}} \in \hat{R}_{\delta} / Q(\delta, L)} \overline{\bar{\chi}} \cdot A^{K}(\eta)
$$

by (5.3.1) and Notation (5.3.4). The following result describes the decomposition of the Langlands subrepresentation of a standard representation (Definition (4.1.19)).

Theorem 5.3.5. Assume $(H, \underline{\gamma})$ is a regular character for $G$. Let $\left(H_{F}, \underline{\gamma_{F}}\right)$ be the limit character constructed in the way mentioned in (5.3.1), with respect to $(H, \gamma)$ and some $\eta \in A_{L}^{F}(\delta)$. We obtain the following.

(a) The decompositions

$$
\begin{aligned}
& X_{G}(H, \underline{\gamma})=m(\delta, \eta) \sum_{\bar{\chi} \in \hat{R}_{\delta}(L) / Q(\delta, L)_{o}} \bar{\chi} \cdot X_{G}\left(H_{F}, \underline{\gamma_{F}}\right), \\
& \bar{X}_{G}(H, \underline{\gamma})=m(\delta, \eta) \sum_{\bar{\chi} \in \hat{R}_{\delta}(L) / Q(\delta, L)_{o}} \bar{\chi} \cdot \bar{X}_{G}\left(H_{F}, \underline{\gamma_{F}}\right) .
\end{aligned}
$$

(b) The set of minimal $K$-types of the $(\mathfrak{g}, K)$-module $\bar{\chi} \cdot X_{G}\left(H_{F}, \underline{\gamma_{F}}\right.$ ) (resp. of $\left.\bar{\chi} \cdot \bar{X}_{G}\left(H_{F}, \underline{\gamma_{F}}\right)\right)$ is $\overline{\bar{\chi}} \cdot A^{K}(\eta)$.

(c) $\overline{\bar{\chi}} \cdot A^{K}(\eta)=A^{K}(\eta)$ if and only if $\overline{\bar{\chi}}=1$.

(d) $\bar{\chi} \cdot X_{G}\left(H_{F}, \underline{\gamma_{F}}\right) \cong X_{G}\left(H_{F}, \underline{\gamma_{F}}\right)$ if and only if $\bar{\chi}=1$.

(e) The multiplicity of $\bar{\chi} \cdot X_{G}\left(\overline{H_{F}}, \underline{\gamma_{F}}\right)$ in $X_{G}(H, \underline{\gamma})$ is $m(\delta, \eta)$.

(f) $m(\delta, \eta)=2^{m}$ for some $m \in \mathbb{N}$.

By Proposition (5.3.3) and Remark (5.2.11) (a), $\left(H_{F}, \underline{\gamma_{F}}\right)$ in Theorem (5.3.5) might not be a final character; however, the module $X_{G}\left(\overline{H_{F}}, \underline{\gamma_{F}}\right)$ can be induced from some final character (Theorem (5.1.14)).

Corollary 5.3.6. We have obtained the classification of tempered representations, (4.1.20).

Proof. Assume that the regular character $(H, \underline{\gamma})$ in Theorem (5.3.5) is such that $X_{G}(H, \gamma)$ is a standard tempered representation, (4.1.16). In this case, $X_{G}(H, \gamma)$ coincides with the Langlands subrepresentation $\bar{X}_{G}(H, \underline{\gamma})$. Theorem (5.3.5) gives a decomposition for it. This proves our assertion.

\section{REFERENCES}

[A-B-V] J. Adams, D. Barbasch, and D. A. Vogan, The Langlands classification and irreducible characters for real reductive groups, Progress in Math., Birkhäuser, Boston, MA, 1992. 
[B-W] A. Borel and N. Wallach, Continuous cohomology, discrete subgroups, and representations of reductive groups, Princeton University Press, Princeton, NJ, 1980.

[C-R] W. C. Curtis and I. Reiner, Representation theory of finite groups and associative algebras, Interscience (Wiley), New York, 1962.

[Green] D. A. Vogan, Representations of real reductive Lie groups, Progress in Math., Birkhäuser, Boston, MA, 1981.

[H] S. Helgason, Differential geometry and symmetric spaces, Academic Press, New York, 1962.

[H-Ch 1] Harish-Chandra, Representations of semi-simple Lie groups I, Trans. Amer. Math. Soc. 75 (1953), 125-243.

[H-Ch 2] __ Representations of semi-simple Lie groups III, Trans. Amer. Math. Soc. 76 (1954), 234-253.

[H-Ch 3] __ Supertempered distributions on a real reductive group, Collected Papers, Vol. IV, Springer-Verlag.

[K] A. W. Knapp, Representation theory of semi-simple groups. An overview based on examples, Princeton University Press, Princeton, NJ, 1986.

[K-Z] A. W. Knapp and G. J. Zuckerman, Classification of irreducible tempered representations of semi-simple Lie groups, Ann. of Math. 116 (1982), 389-501.

[L] J. Lepowsky, Algebraic results on representations of semi-simple Lie groups, Trans. Amer. Math. Soc. 76 (1973), 1-44.

[M] I. Mirković, Classification of irreducible tempered representations of semi-simple groups, Ph.D. thesis, Univ. of Utah, 1986.

[S-V] B. Speh and D. A. Vogan, Reducibility of generalized principal series representations, Acta Math. 145 (1980), 227-299.

[V-I] D. A. Vogan, The algebraic structure of the representations of semi-simple Lie groups I, Ann. of Math. 109 (1976), 1-60.

[V-II] _ Irreducible characters of semi-simple Lie groups I, Duke Math. J. 46 (1979), 61-108.

[W-1] N. Wallach, Representations of semi-simple Lie groups and Lie algebras, Proc. Canad. Math. Cong. (1977), 154-245.

[W-2] — Real reductive groups I, Academic Press, New York, 1988.

[W-3] _ Real reductive groups II, Academic Press, New York, 1992.

Instituto de Matemáticas, U. N. A. M., Mexico 04510, México

E-mail address: garnica@redvax 1.dgsca.unam.mx 